\title{
Metal-Catalyzed di-tert-Butylsilylene Transfer: Synthesis and Reactivity of Silacyclopropanes
}

\author{
Jelena Cirakovic, Tom G. Driver, and K. A. Woerpel* \\ Department of Chemistry, University of California, \\ Irvine, California, 92697-2025
}

Contents:

\section{Supporting Information}

I. Preparation of Alkene $11 \quad s-2$

II. Silacyclopropane Formation $s-3$

III. Synthesis of Oxasilacyclopentanes from Alkenes $s-4$

IV. Construction of 1,3-Diols from Oxasilacyclopentanes $s-10$

V. References $s-12$

VI. Spectral Data $s-13$

General. ${ }^{1} \mathrm{H}$ NMR and ${ }^{13} \mathrm{C}$ NMR spectra were recorded at ambient temperature using Bruker DRX 500 or DRX $400 \mathrm{MHz}$ spectrometers. The data are reported as follows: chemical shift in ppm from internal tetramethylsilane standard on the $\delta$ scale, multiplicity ( $\mathrm{br}=$ broad, $\mathrm{s}=$ singlet, $\mathrm{d}=$ doublet, $\mathrm{t}=$ triplet, $\mathrm{q}=$ quartet, $\mathrm{m}=$ multiplet $)$, coupling constants $(\mathrm{Hz})$, and integration. High resolution mass spectra were acquired on a VG Analytical 7070E or Fisons Autospec spectrometer, and were obtained by peak matching. Microanalyses were performed by Atlantic Microlab, Atlanta, GA. GC analyses were performed on an Agilent $6850 \mathrm{GC}$ system gas chromatograph. Analytical thin layer chromatography was performed on EM Reagents $0.25 \mathrm{~mm}$ silica gel $60-\mathrm{F}$ plates. Liquid chromatography was performed using forced flow (flash chromatography) of the indicated solvent system on EM silica gel $\left(\mathrm{SiO}_{2}\right) 60$ (230-400) mesh, or gravity column chromatography on Fisher neutral alumina Broeckman activity I (60 - 325) mesh. HPLC Osmonics polypropylene 0.45 micron filters were used. The reactions were carried out under an atmosphere of argon, in glassware which had been flame dried under vacuum, or in an Innovative Technologies nitrogen atmosphere dry box. Unless otherwise noted, all reagents were commercially obtained and, where appropriate, purified prior to use. Toluene, $\mathrm{Et}_{2} \mathrm{O}$, and $\mathrm{CH}_{2} \mathrm{Cl}_{2}$ were dried by filtration through alumina according to the procedure of Grubbs. ${ }^{1}$ Metal salts and cyclohexene di-tert-butyl silacyclopropane 1 were stored in an Innovative Technologies nitrogen atmosphere dry box. 


\section{Preparation of Alkene 11}

Spectral data for compounds $\mathbf{2 a - j}$, and 3-10 have already been reported. ${ }^{2}$

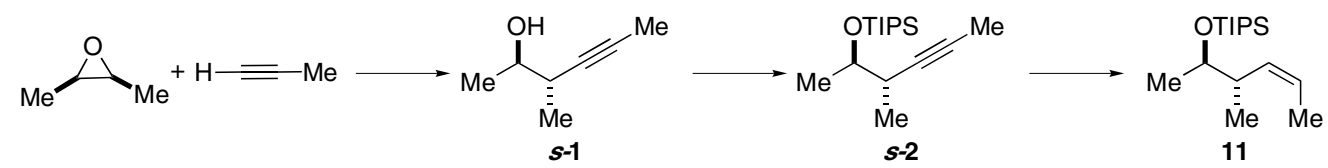

Alkene 11. To a cooled $\left(-78{ }^{\circ} \mathrm{C}\right)$ solution of $n$-BuLi $(10.40 \mathrm{~mL}$ of $2.0 \mathrm{M}$ solution of $n$ BuLi in hexanes, $20.80 \mathrm{mmol})$ in THF $(80 \mathrm{~mL})$ was introduced propyne until the solution became milky white. $\mathrm{BF}_{3} \cdot \mathrm{Et}_{2} \mathrm{O}(3.16 \mathrm{~mL}, 27.10 \mathrm{mmol})$ was then added dropwise. After 10 minutes, cis-2,3-epoxybutane was added dropwise and the mixture was left stirring at $-78{ }^{\circ} \mathrm{C}$ for 45 minutes. The mixture was then quenched with saturated aqueous $\mathrm{NH}_{4} \mathrm{Cl}$ and allowed to reach room temperature. The two layers that formed were separated. Water layer was extracted once with MTBE $(20 \mathrm{~mL})$. The combined organic extracts were dried over $\mathrm{Na}_{2} \mathrm{SO}_{4}$ and concentrated. Purification by flash chromatography (pentane: $\left.\mathrm{Et}_{2} \mathrm{O}=91: 9\right)$ yielded the product $\boldsymbol{s}-1$ as a colorless oil $(1.450 \mathrm{~g}, 92 \%)$ : ${ }^{1} \mathrm{H}$ NMR $\left(\mathrm{CDCl}_{3}, 400 \mathrm{MHz}\right) \delta 3.57(\mathrm{q}, J=6.0,1 \mathrm{H}), 2.39(\mathrm{~m}, 1 \mathrm{H}), 1.98(\mathrm{br} \mathrm{s}, 1 \mathrm{H}), 1.82(\mathrm{~d}, J=2.4$, $3 \mathrm{H}), 1.22(\mathrm{~d}, J=6.0,3 \mathrm{H}), 1.16(\mathrm{~d}, J=7.2,3 \mathrm{H}) ;{ }^{13} \mathrm{C} \mathrm{NMR}\left(\mathrm{CDCl}_{3}, 100 \mathrm{MHz}\right) \delta 79.8$, 78.7, 70.9, 34.9, 20.8, 17.6, 3.5; IR (thin film) 3406, 2975, 2921, 1451, 1376, $1101 \mathrm{~cm}^{-1}$.

Alcohol $s-1$ obtained was then further utilized in the next step. To a cooled $(-78$ $\left.{ }^{\circ} \mathrm{C}\right)$ suspension of potassium hydride $(0.299 \mathrm{~g}, 7.455 \mathrm{mmol})$ and $18-\mathrm{c}-6(0.002 \mathrm{~g}, 0.007$ mmol) in THF $(10 \mathrm{~mL})$ was added $\boldsymbol{s}-\mathbf{1}(0.497 \mathrm{~g}, 4.430 \mathrm{mmol})$ as a solution in THF (5 $\mathrm{mL})$. After 10 minutes, TIPSCl $(1.39 \mathrm{~mL}, 6.49 \mathrm{mmol})$ was added and the mixture was left to reach room temperature overnight. The reaction mixture was then diluted with $\mathrm{Et}_{2} \mathrm{O}(20 \mathrm{~mL})$, the reagents were quenched with $0.01 \mathrm{M} \mathrm{HCl}(5 \mathrm{~mL})$, and the layers were separated. The organic extract was washed once with brine $(20 \mathrm{~mL})$, dried over $\mathrm{Na}_{2} \mathrm{SO}_{4}$, and concentrated. Purification by gravity alumina column chromatography (pentane) afforded the product $\boldsymbol{s}-\mathbf{2}$ as a colorless oil $(1.180 \mathrm{~g}, 99 \%)$ : ${ }^{1} \mathrm{H} \mathrm{NMR}\left(\mathrm{CDCl}_{3}, 400 \mathrm{MHz}\right) \delta$ 4.04 (dq, $J=6.2,4.3,1 \mathrm{H}), 2.57$ (qdd, $J=7.0,4.3,2.4,1 \mathrm{H}), 1.78(\mathrm{~d}, J=2.4,3 \mathrm{H}), 1.18$ $(\mathrm{d}, J=6.2,3 \mathrm{H}), 1.11(\mathrm{~d}, J=7.0,3 \mathrm{H}), 1.06(\mathrm{~s}, 18 \mathrm{H}$ and $3 \mathrm{H}) ;{ }^{13} \mathrm{C} \mathrm{NMR}\left(\mathrm{CDCl}_{3}, 100\right.$ MHz) $\delta 81.7,76.7,70.6,35.6,19.0,18.6,18.1,13.9,12.4$; IR (thin film) 2943, 2867, $1463,1378,1108,1016 \mathrm{~cm}^{-1}$; HRMS (CI/isobutene) $\mathrm{m} / z$ calcd for $\mathrm{C}_{16} \mathrm{H}_{33} \mathrm{OSi}(\mathrm{M}+\mathrm{H})^{+}$ 269.2301, found 269.2302. Anal. Calcd for $\mathrm{C}_{16} \mathrm{H}_{32} \mathrm{OSi}$ : C, 71.57; H, 12.01. Found $\mathrm{C}$, $71.61 ; \mathrm{H}, 12.10$.

TIPS ether $\boldsymbol{s}-\mathbf{2}$ obtained was reduced in the following manner: alkyne $\boldsymbol{s - 2}(0.219$ $\mathrm{g}, 0.816 \mathrm{mmol})$ was added to a suspension of Lindlar catalyst $\left(\mathrm{Pd} \cdot \mathrm{CaCO}_{3}(\mathrm{~Pb}), 0.074 \mathrm{~g}\right.$, $0.030 \mathrm{mmol}$ of Pd) in benzene $(10 \mathrm{~mL})$. A balloon of hydrogen was attached to the flask through the septum and the reaction mixture was left stirring overnight. The mixture was then filtered through a plug of Celite and concentrated. Purification by flash chromatography (pentane) afforded the product $\mathbf{1 1}$ as a colorless oil $(0.216 \mathrm{~g}, 98 \%):{ }^{1} \mathrm{H}$ NMR (500 MHz, CDCl $) \delta 5.47(\mathrm{~m}, 1 \mathrm{H}), 5.31(\mathrm{~m}, 1 \mathrm{H}), 3.90(\mathrm{qd}, J=6.2,3.8,1 \mathrm{H}), 2.62$ $(\mathrm{m}, 1 \mathrm{H}), 1.63(\mathrm{dd}, J=6.8,1.7,3 \mathrm{H}), 1.09(\mathrm{~s}, 18 \mathrm{H}$ and $6 \mathrm{H}), 0.99(\mathrm{~d}, J=6.8,3 \mathrm{H}) ;{ }^{13} \mathrm{C}$ NMR (125 MHz, $\left.\mathrm{CDCl}_{3}\right) \delta 133.4,123.6,71.6,38.7,19.7,18.2,15.2,13.0,12.7$; IR (thin film) 3014, 2961, 1464, 1374, 1104, $883 \mathrm{~cm}^{-1}$; HRMS (CI/isobutene) $\mathrm{m} / z$ calcd for $\mathrm{C}_{13} \mathrm{H}_{27} \mathrm{OSi}(\mathrm{M}-i-\mathrm{Pr})^{+}$227.1831, found 227.1827. Anal. Calcd for $\mathrm{C}_{16} \mathrm{H}_{34} \mathrm{OSi}: \mathrm{C}, 71.04$; $\mathrm{H}, 12.67$. Found $\mathrm{C}, 71.15 ; \mathrm{H}, 12.76$. 


\section{Silacyclopropane formation}

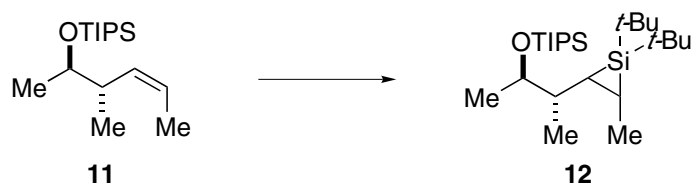

Silacyclopropane 12. To a cooled $\left(-20^{\circ} \mathrm{C}\right)$ suspension of $\operatorname{AgOTf}(0.001 \mathrm{~g}, 0.004 \mathrm{mmol})$ in $0.7 \mathrm{~mL}$ of $0.019 \mathrm{M}$ solution of $\mathrm{PhSiMe}_{3}$ in $d_{8}$-toluene, contained in an NMR tube, was added alkene $11(0.049 \mathrm{~g}, 0.181 \mathrm{mmol})$. The solution of cyclohexene silacyclopropane $\mathbf{1}$ $(0.034 \mathrm{~g}, 0.152 \mathrm{mmol})$ in $0.3 \mathrm{~mL}$ of $0.019 \mathrm{M}$ solution of $\mathrm{PhSiMe}_{3}$ in $d_{8}$-toluene was cooled to $-20{ }^{\circ} \mathrm{C}$ and added to the NMR tube. After $12 \mathrm{~h}$, the reagents were quenched with TMEDA $(0.010 \mathrm{~mL})$ and the reaction mixture was analyzed by ${ }^{1} \mathrm{H}$ and ${ }^{29} \mathrm{Si} \mathrm{NMR}$ spectroscopy. Silacyclopropane $\mathbf{1 2}$ was formed in 79\% as a 89:11 mixture of isomers. The ${ }^{29} \mathrm{Si}$ NMR spectrum was obtained to verify the formation of product silacyclopropane and to determine the diastereoselectivity of the reaction. The yield of the reaction was obtained through comparison of the area of the standard $\mathrm{PhSiMe}_{3}$ peak at $0.20 \mathrm{ppm}$ and the area of the $t$-Bu peak of the product:

\begin{tabular}{ccccc}
\hline $\begin{array}{c}{ }^{29} \text { Si NMR peaks (ppm) } \\
\text { rel. external TMS standard }\end{array}$ & $\begin{array}{c}\text { Relative ratio } \\
\text { of }{ }^{29} \text { Si NMR peaks }\end{array}$ & $\begin{array}{c}\text { Product 12 } t \text { Bu } \\
\text { ppm (area) }\end{array}$ & $\begin{array}{c}\text { Standard } \\
\text { (equiv., area) }^{\mathrm{b}}\end{array}$ & \%yield ${ }^{\mathrm{b}}$ \\
\hline$-51.02,-54.02$ & $11: 89$ & $1.15(4.85)^{\mathrm{c}}$ & $\begin{array}{c}\mathrm{PhSiMe}_{3} \\
(0.129,1.00)\end{array}$ & 79 \\
\hline
\end{tabular}

a The ${ }^{29} \mathrm{Si} \mathrm{NMR} \mathrm{spectrum} \mathrm{of} 1$ contains one peak at $-54.6 \mathrm{ppm} .{ }^{\mathrm{b}}$ As determined by ${ }^{1} \mathrm{H}$ NMR analysis of product mixture relative to an interal standard of $\mathrm{PhSiMe}_{3}$. Yield $=$ (equiv. standard) (area of $t$-bu peak)/(area of standard). ${ }^{\mathrm{C}} t$-Bu peak with smaller area used in the yield calculation.

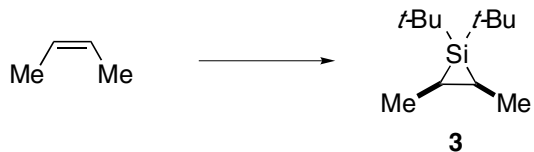

2,3-cis-Dimethyl-1,1-di-tert-butylsilacyclopropane (3). ${ }^{3}$ Cold $\left(-78{ }^{\circ} \mathrm{C}\right)$ cis-butene (ca. $1 \mathrm{~mL}$, excess), was added by cannula to a cooled $\left(0{ }^{\circ} \mathrm{C}\right)$ Schlenk flask that contained a suspension of $\mathrm{AgOC}(\mathrm{O}) \mathrm{CF}_{3}(0.007 \mathrm{~g}, 0.032 \mathrm{mmol})$ in $\mathrm{CH}_{2} \mathrm{Cl}_{2}(12 \mathrm{~mL})$. A solution of cyclohexene silacyclopropane $1(0.709 \mathrm{~g}, 3.159 \mathrm{mmol})$ in $\mathrm{CH}_{2} \mathrm{Cl}_{2}(3 \mathrm{~mL})$ was added dropwise, and the reaction mixture was allowed to reach room temperature overnight. The crude reaction mixture was filtered through Celite 545 under an atmosphere of argon (Schlenk filtration) and the cake was washed four times with freshly distilled hexanes (5 $\times 10 \mathrm{~mL}$ ). The filtrate was concentrated in vacuo. Purification by bulb-to-bulb distillation afforded the silacyclopropane 3 as a colorless oil $(0.418 \mathrm{~g}, 67 \%)$ : ${ }^{1} \mathrm{H}$ NMR $\left(400 \mathrm{MHz} \mathrm{CDCl}_{3}\right) \delta 1.28(\mathrm{~m}, 6 \mathrm{H}), 1.19(\mathrm{~s}, 9 \mathrm{H}), 1.02(\mathrm{~s}, 9 \mathrm{H}), 0.94(\mathrm{~m}, 2 \mathrm{H}) ;{ }^{13} \mathrm{C} \mathrm{NMR}$ $\left(125 \mathrm{MHz} \mathrm{CDCl}_{3}\right) \delta 31.5,29.8,9.9,9.5$; IR (thin film): 2988, 2860, 1474, 1388, 1364 $\mathrm{cm}^{-1}$; HRMS (CI/isobutene) $m / z$ calcd for $\mathrm{C}_{12} \mathrm{H}_{26} \mathrm{Si}(\mathrm{M})^{+}$198.1804, found 198.1795.

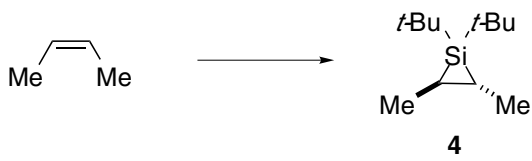


2,3-trans-Dimethyl-1,1-di-tert-butylsilacyclopropane (4). ${ }^{3}$ The procedure for 3 was followed using ca. $1 \mathrm{~mL}$ of trans-2-butene, $0.800 \mathrm{~g}$ of cyclohexene silacyclopropane 1 (3.564 mmol), and $0.008 \mathrm{~g}$ of $\mathrm{AgOC}(\mathrm{O}) \mathrm{CF}_{3}(0.036 \mathrm{mmol})$. Purification by bulb-to-bulb distillation afforded the silacyclopropane 4 as a colorless oil $(0.565 \mathrm{~g}, 80 \%)$ : ${ }^{1} \mathrm{H} \mathrm{NMR}$ $\left(400 \mathrm{MHz}, \mathrm{CDCl}_{3}\right) \delta 1.33(\mathrm{~m}, 6 \mathrm{H}), 1.12(\mathrm{~s}, 18 \mathrm{H}), 0.51(\mathrm{~m}, 2 \mathrm{H}) ;{ }^{13} \mathrm{C}$ NMR $(100 \mathrm{MHz}$, $\left.\mathrm{CDCl}_{3}\right) \delta 30.7,19.6,16.9,16.4$; IR (thin film): 2966, 2859, 1473, 1455, 1388, $1363 \mathrm{~cm}^{-1}$; HRMS (CI/isobutene) $m / z$ calcd for $\mathrm{C}_{12} \mathrm{H}_{26} \mathrm{Si}(\mathrm{M})^{+}$198.1804, found 198.1808.

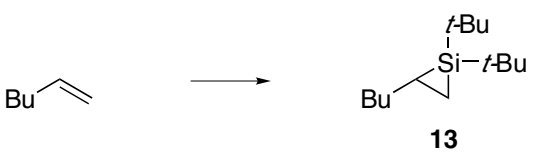

2-Butyl-1,1-di-tert-butyl silacyclopropane (13) To a cooled $\left(0{ }^{\circ} \mathrm{C}\right)$ suspension of $\mathrm{AgOC}(\mathrm{O}) \mathrm{CF}_{3}(0.004 \mathrm{~g}, 0.018 \mathrm{mmol})$ in $\mathrm{CH}_{2} \mathrm{Cl}_{2}(10 \mathrm{~mL})$ was added 1-hexene $(0.90 \mathrm{~mL}$, $7.20 \mathrm{mmol})$. A solution of $1(0.814 \mathrm{~g}, 3.626 \mathrm{mmol})$ in $\mathrm{CH}_{2} \mathrm{Cl}_{2}(2 \mathrm{~mL})$ was then added dropwise and the reaction mixture was allowed to reach room temperature overnight. The reaction mixture was filtered through Celite 545 under an atmosphere of argon (Schlenk filtration) and the cake was washed with freshly distilled hexanes $(5 \times 10 \mathrm{~mL})$. The filtrate was then concentrated in vacuo. Purification by bulb-to-bulb distillation afforded the product as a colorless oil $(0.739 \mathrm{~g}, 90 \%)$ : ${ }^{1} \mathrm{H}$ NMR $\left(500 \mathrm{MHz}, \mathrm{CDCl}_{3}\right) \delta$ $1.66-1.61(\mathrm{~m}, 1 \mathrm{H}), 1.58-1.52(\mathrm{~m}, 1 \mathrm{H}), 1.42(\mathrm{~m}, 1 \mathrm{H}), 1.37-1.29(\mathrm{~m}, 3 \mathrm{H}), 1.13(\mathrm{~s}, 9 \mathrm{H})$, 1.05 (s, 9H), 0.89 (t, $J=7.0,3 \mathrm{H}), 0.76(\mathrm{ddd}, J=16.5,12.1,8.4,1 \mathrm{H}), 0.66(\mathrm{dd}, J=12.1$, $10.3,1 \mathrm{H}), 0.35(\mathrm{dd}, J=10.3,8.7,1 \mathrm{H}) ;{ }^{13} \mathrm{C} \mathrm{NMR}\left(125 \mathrm{MHz}, \mathrm{CDCl}_{3}\right) \delta 34.7,34.1,31.8$, 30.7, 29.8, 22.6, 18.9, 14.3, 14.0, 3.2; IR (thin film): 2966, 2857, 1474, 1459, 1388, $1363,1012 \mathrm{~cm}^{-1}$; HRMS (EI) $\mathrm{m} / z$ calcd for $\mathrm{C}_{14} \mathrm{H}_{30} \mathrm{Si}(\mathrm{M})^{+} 226.2117$, found 226.2116.

\section{Synthesis of oxasilacyclopentanes from alkenes}

Spectral data for compounds 14a-g have already been reported. ${ }^{2}$

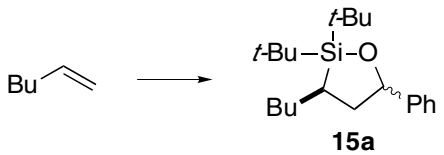

Oxasilacyclopentane 15a. To a cooled $\left(0{ }^{\circ} \mathrm{C}\right)$ Schlenk flask that contained the suspension of $\mathrm{AgOC}(\mathrm{O}) \mathrm{CF}_{3}(0.004 \mathrm{~g}, 0.018 \mathrm{mmol})$ in $\mathrm{CH}_{2} \mathrm{Cl}_{2}(5 \mathrm{~mL})$ was added 1hexene $(0.070 \mathrm{ml}, 0.559 \mathrm{mmol})$ and the reaction mixture was stirred for 10 minutes. A solution of 1 ( $0.064 \mathrm{~g}, 0.285 \mathrm{mmol})$ in $\mathrm{CH}_{2} \mathrm{Cl}_{2}(2 \mathrm{~mL})$ was added dropwise and the mixture was left stirring for $2 \mathrm{~h}$. The mixture was then cooled to $-78{ }^{\circ} \mathrm{C}$, and benzaldehyde $(0.060 \mathrm{ml}, 0.590 \mathrm{mmol})$ was then added followed by $\mathrm{ZnBr}_{2}(0.018 \mathrm{mg}$, $0.080 \mathrm{mmol}$ ) and the mixture was allowed to reach room temperature overnight. The mixture was then diluted with $\mathrm{CH}_{2} \mathrm{Cl}_{2}(5 \mathrm{~mL})$ and washed with brine $(5 \mathrm{~mL})$. The water layer was then extracted with $\mathrm{CH}_{2} \mathrm{Cl}_{2}(5 \mathrm{~mL})$. The combined organic extracts were filtered through a cotton plug and concentrated to afford the product as a 65:35 mixture of diastereomers as determined by GC analysis. Purification by flash chromatography (pentane:EtOAc = 99:1) afforded the product as a colorless oil $(0.062 \mathrm{~g}, 73 \%)$ : IR (thin 
film) 3028, 2930, 1473, 1458, 1041, $824 \mathrm{~cm}^{-1}$; HRMS (CI/isobutene) $\mathrm{m} / \mathrm{z}$ calcd for $\mathrm{C}_{21} \mathrm{H}_{35} \mathrm{OSi}(\mathrm{M}-\mathrm{H})^{+}$331.2457, found 331.2442. Anal. Calcd for $\mathrm{C}_{21} \mathrm{H}_{36} \mathrm{OSi}: \mathrm{C}, 75.84 ; \mathrm{H}$, 10.91. Found $\mathrm{C}, 75.82 ; \mathrm{H}, 11.06$. Selected data for major diastereomer: ${ }^{1} \mathrm{H}$ NMR (400 $\left.\mathrm{MHz}, \mathrm{CDCl}_{3}\right) \delta 7.28(\mathrm{~m}, 5 \mathrm{H}), 4.76(\mathrm{dd}, J=11.2,4.0,1 \mathrm{H}), 2.36(\mathrm{ddd}, J=11.8,6.1,3.9$, $1 \mathrm{H}), 2.04(\mathrm{dd}, J=7.6,6.8,1 \mathrm{H}), 1.67(\mathrm{~m}, 2 \mathrm{H}), 1.32(\mathrm{~m}, 5 \mathrm{H}), 1.08(\mathrm{~s}, 9 \mathrm{H}), 1.02(\mathrm{~s}, 9 \mathrm{H})$, $0.83(\mathrm{t}, J=7.0,3 \mathrm{H}) ;{ }^{13} \mathrm{C}$ NMR $\left(125 \mathrm{MHz}, \mathrm{CDCl}_{3}\right) \delta 128.2,126.8,125.5,125.0,79.3$, 43.5, 33.5, 29.8, 28.6, 28.5, 28.1, 25.9, 22.7, 14.1. Selected data for minor diastereomer: ${ }^{1} \mathrm{H}$ NMR (400 MHz, $\left.\mathrm{CDCl}_{3}\right) \delta 7.28(\mathrm{~m}, 5 \mathrm{H}), 5.02(\mathrm{t}, J=6.6,1 \mathrm{H}), 1.66(\mathrm{~m}, 2 \mathrm{H}), 1.32(\mathrm{~m}$, $6 \mathrm{H}), 1.07(\mathrm{~s}, 9 \mathrm{H}), 0.98(\mathrm{~s}, 9 \mathrm{H}) ;{ }^{13} \mathrm{C}$ NMR $\left(125 \mathrm{MHz}, \mathrm{CDCl}_{3}\right) \delta 128.2,128.1,126.8$, $126.7,78.1,47.8,33.6,33.0,30.5,27.8,27.7,13.4$

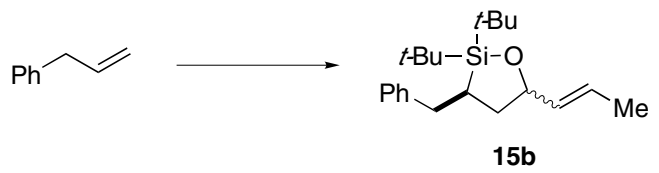

Oxasilacyclopentane (15b). To a cooled $\left(-15^{\circ} \mathrm{C}\right)$ suspension of $\mathrm{AgOC}(\mathrm{O}) \mathrm{CF}_{3}(0.003 \mathrm{~g}$, $0.015 \mathrm{mmol}$ ) in $2 \mathrm{~mL}$ of toluene was added, dropwise, a solution containing cyclohexene silacyclopropane $1(0.048 \mathrm{~g}, 0.214 \mathrm{mmol})$ and $0.036 \mathrm{~mL}$ of allylbenzene $(0.267 \mathrm{mmol})$ in $0.5 \mathrm{~mL}$ of toluene. After 90 minutes, the resultant dark suspension was added, dropwise, to a cold $\left(-78{ }^{\circ} \mathrm{C}\right)$ suspension of $\mathrm{ZnI}_{2}(0.014 \mathrm{~g}, 0.446 \mathrm{mmol})$ in $0.7 \mathrm{~mL}$ of toluene. Freshly distilled crotonaldehyde $(0.037 \mathrm{~mL}, 0.446 \mathrm{mmol})$ was added dropwise. The resultant suspension was allowed to slowly warm to $22{ }^{\circ} \mathrm{C}$. After $5 \mathrm{~h}$, the reaction mixture was passed through silanized silica gel and concentrated in vacuo to afford $\mathbf{1 5 b}$ as a 69:31 mixture of diastereomers as determined by ${ }^{1} \mathrm{H}$ NMR analysis. Purification by flash chromatography (hexanes) afforded the product $\mathbf{1 5 b}$, as a colorless oil $(0.055 \mathrm{~g}$, $78 \%$ ). The regioselectivity was determined from 2D gCOSY NMR spectroscopy. Major diastereomer: ${ }^{1} \mathrm{H}$ NMR $\left(500 \mathrm{MHz}, \mathrm{CDCl}_{3}\right) \delta 7.26(\mathrm{~m}, 5 \mathrm{H}), 5.61(\mathrm{~m}, 1 \mathrm{H}), 5.47$ (tdd, $J=$ $10.8,7.5,1.5,1 \mathrm{H}), 4.57(\mathrm{td}, J=7.6,3.4,1 \mathrm{H}), 3.13(\mathrm{dd}, J=13.9,3.4,1 \mathrm{H}), 2.73(\mathrm{~m}, 1 \mathrm{H})$, $1.90(\mathrm{~m}, 1 \mathrm{H}), 1.69(\mathrm{~m}, 5 \mathrm{H}), 1.09(\mathrm{~s}, 9 \mathrm{H}), 1.06(\mathrm{~s}, 9 \mathrm{H}) ;{ }^{13} \mathrm{C} \mathrm{NMR}\left(125 \mathrm{MHz}, \mathrm{CDCl}_{3}\right) \delta$ 143.2, 134.1, 133.7, 128.3, 125.8, 125.7, 77.9, 28.6, 28.4, 24.4, 22.1, 21.4, 21.3, 20.2, 17.5. Selected data for minor diastereomer: ${ }^{1} \mathrm{H}$ NMR $\left(500 \mathrm{MHz}, \mathrm{CDCl}_{3}\right) \delta 4.12(\mathrm{ddd}, J=$ 13.7, 9.5, 3.7, 1H), $1.40(\mathrm{~m}, 1 \mathrm{H}), 1.07(\mathrm{~s}, 9 \mathrm{H}), 1.03(\mathrm{~s}, 9 \mathrm{H}) ;{ }^{13} \mathrm{C} \mathrm{NMR}\left(125 \mathrm{MHz}, \mathrm{CDCl}_{3}\right)$ $\delta 143.3,128.2,126.0,78.7,28.08,28.03,27.6,17.6$; IR (thin film) 3026, 2856, 1472, 1003, $822 \mathrm{~cm}^{-1}$; HRMS (EI) $m / z$ calcd for $\mathrm{C}_{21} \mathrm{H}_{34} \mathrm{OSi}(\mathrm{M})^{+} 330.2379$, found 330.2362. Anal. Calcd for $\mathrm{C}_{21} \mathrm{H}_{34} \mathrm{OSi}$ : C, 76.30; H, 10.37. Found C, 75.94; H, 10.40.

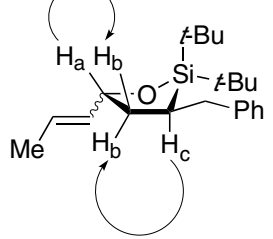

Coupling (COSY cross peaks) observed between (1) $\mathrm{H}_{\mathrm{a}}$ and $\mathrm{H}_{\mathrm{b}}$ and (2) $\mathrm{H}_{\mathrm{b}}$ and $\mathrm{H}_{\mathrm{c}}$ Note: no coupling observed between $\mathrm{H}_{\mathrm{a}}$ and $\mathrm{H}_{\mathrm{c}}$

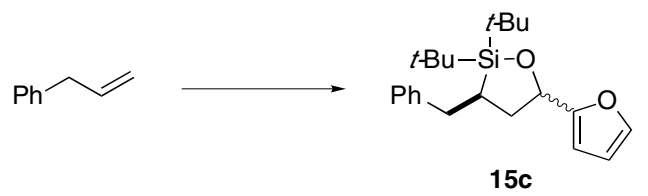


Oxasilacyclopentane (15c). The procedure for $\mathbf{1 5 b}$ was followed using $0.060 \mathrm{~g}$ of cyclohexene silacyclopropane $1(0.267 \mathrm{mmol}), 0.042 \mathrm{~mL}$ of allylbenzene $(0.321 \mathrm{mmol})$, $0.003 \mathrm{~g}$ of $\mathrm{AgOCOCF}_{3}(0.01 \mathrm{mmol}), 0.051 \mathrm{~mL}$ of freshly distilled furfural $(0.534 \mathrm{mmol})$, and $0.017 \mathrm{~g}$ of $\mathrm{ZnI}_{2}(0.0534 \mathrm{mmol})$. After $5 \mathrm{~h}$, the reaction mixture was passed through silanized silica gel and concentrated in vacuo to afford 15c as a 64:36 mixture of diastereomers as determined by ${ }^{1} \mathrm{H}$ NMR analysis. Purification using flash chromatography (hexanes) afforded the product $15 \mathrm{c}(0.078 \mathrm{~g}, 80 \%)$. The regioselectivity was determined from 2D gCOSY NMR spectroscopy. IR (thin film) 2932, 2857, 1474, $1009,823 \mathrm{~cm}^{-1}$; HRMS (EI) $m / z$ calcd for $\mathrm{C}_{22} \mathrm{H}_{33} \mathrm{O}_{2} \mathrm{Si}(\mathrm{M}+\mathrm{H})^{+} 357.2250$, found 357.2253. Major diastereomer: ${ }^{1} \mathrm{H}$ NMR $\left(500 \mathrm{MHz}, \mathrm{CDCl}_{3}\right) \delta 7.26(\mathrm{~m}, 6 \mathrm{H}), 6.29(\mathrm{dd}, J$ $=3.2,1.8,1 \mathrm{H}), 6.23(\mathrm{dd}, J=4.5,1.8,1 \mathrm{H}), 4.74(\mathrm{dd}, J=11.4,4.4,1 \mathrm{H}), 3.18(\mathrm{~m}, 1 \mathrm{H})$, $2.80(\mathrm{~m}, 1 \mathrm{H}), 2.55(\mathrm{~m}, 1 \mathrm{H}), 1.88(\mathrm{~m}, 1 \mathrm{H}), 1.72(\mathrm{~m}, 1 \mathrm{H}), 1.19(\mathrm{~s}, 9 \mathrm{H}), 1.13(\mathrm{~s}, 9 \mathrm{H}) ;{ }^{13} \mathrm{C}$ NMR $\left(125 \mathrm{MHz}, \mathrm{CDCl}_{3}\right) \delta 156.2,143.1,141.9,128.4,128.2,125.9,109.9,105.9,72.8$, $38.2,36.4,27.92,27.90,27.5,21.5,21.4$. Selected data for minor diastereomer: ${ }^{1} \mathrm{H}$ $\operatorname{NMR}\left(500 \mathrm{MHz}, \mathrm{CDCl}_{3}\right) \delta 6.23($ br s, $1 \mathrm{H}), 6.00(\mathrm{~d}, J=3.2,1 \mathrm{H}), 5.22(\mathrm{dd}, J=7.7,2.2$, $1 \mathrm{H}), 2.21$ (ddd, $J=10.9,8.0,2.4,1 \mathrm{H}), 1.18(\mathrm{~s}, 9 \mathrm{H}), 0.96(\mathrm{~s}, 9 \mathrm{H}) ;{ }^{13} \mathrm{C}$ NMR $(125 \mathrm{MHz}$, $\left.\mathrm{CDCl}_{3}\right) \delta 156.8,143.0,141.8,128.4,128.2,109.7,105.5,71.6,36.4,35.5,28.3,28.2$, $24.9,22.1,20.3$.

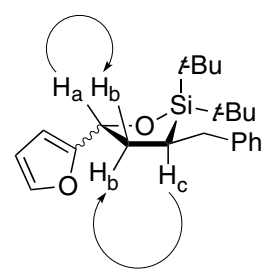

Coupling (COSY cross peaks) observed between (1) $\mathrm{H}_{\mathrm{a}}$ and $\mathrm{H}_{\mathrm{b}}$ and (2) $\mathrm{H}_{\mathrm{b}}$ and $\mathrm{H}_{\mathrm{c}}$ Note: no coupling observed between $\mathrm{H}_{\mathrm{a}}$ and $\mathrm{H}_{\mathrm{c}}$

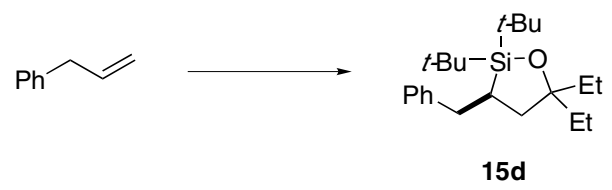

Oxasilacyclopentane (15d). The procedure for 15b was followed using $0.020 \mathrm{~g}$ of cyclohexene silacyclopropane $1(0.089 \mathrm{mmol}), 0.014 \mathrm{~mL}$ of allylbenzene $(0.106 \mathrm{mmol})$, $0.001 \mathrm{~g}$ of $\mathrm{AgOCOCF}_{3}(0.004 \mathrm{mmol}), 0.019 \mathrm{~mL}$ of freshly distilled 3-pentanone $(0.178$ $\mathrm{mmol})$, and $0.006 \mathrm{~g}$ of $\mathrm{ZnI}_{2}(0.017 \mathrm{mmol})$. Purification using flash chromatography (hexanes) afforded the product 15d $(0.020 \mathrm{~g}, 66 \%)$ : ${ }^{1} \mathrm{H} \mathrm{NMR}\left(500 \mathrm{MHz}, \mathrm{CDCl}_{3}\right) \delta 7.24$ $(\mathrm{m}, 5 \mathrm{H}), 3.14(\mathrm{dd}, J=13.9,3.7,1 \mathrm{H}), 2.74(\mathrm{dd}, J=13.9,11.5,1 \mathrm{H}), 1.76(\mathrm{~m}, 3 \mathrm{H}), 1.47(\mathrm{~m}$, $3 \mathrm{H}), 1.34(\mathrm{~m}, 1 \mathrm{H}), 1.14(\mathrm{~s}, 9 \mathrm{H}), 1.08(\mathrm{~s}, 9 \mathrm{H}), 0.80(\mathrm{q}, J=7.6,6 \mathrm{H}) ;{ }^{13} \mathrm{C}$ NMR $(125 \mathrm{MHz}$, $\left.\mathrm{CDCl}_{3}\right) \delta 143.5,128.3,128.2,125.7,82.9,42.6,36.6,32.4,29.5,28.7,28.6,25.3,21.8$, 20.5, 9.0, 8.3; IR (thin film) 2933, 2856, 1474, 989, $703 \mathrm{~cm}^{-1}$; HRMS (EI) $\mathrm{m} / z$ calcd for $\mathrm{C}_{22} \mathrm{H}_{37} \mathrm{OSi}(\mathrm{M}-\mathrm{H})^{+} 345.2614$, found 345.2618. 


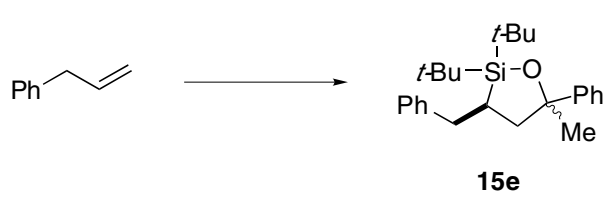

Oxasilacyclopentane (15e). The procedure for $15 \mathrm{~b}$ was followed using $0.020 \mathrm{~g}$ of cyclohexene silacyclopropane $1(0.089 \mathrm{mmol}), 0.014 \mathrm{~mL}$ of allylbenzene $(0.106 \mathrm{mmol})$, $0.001 \mathrm{~g}$ of $\mathrm{AgOCOCF}_{3}(0.004 \mathrm{mmol}), 0.021 \mathrm{~mL}$ of freshly distilled acetophenone $(0.178$ $\mathrm{mmol})$, and $0.006 \mathrm{~g}$ of $\mathrm{ZnI}_{2}(0.018 \mathrm{mmol})$. After $5 \mathrm{~h}$, the reaction mixture was passed through silanized silica gel and concentrated in vacuo to afford 15e as a 53:47 mixture of diastereomers as determined by ${ }^{1} \mathrm{H}$ NMR analysis. Purification using flash chromatography (hexanes) afforded the product 15e $(0.033 \mathrm{~g}, 96 \%)$ : IR (thin film) 3086 , 2931, 1602, 1471, $953 \mathrm{~cm}^{-1}$; HRMS (CI/isobutane) $\mathrm{m} / \mathrm{z}$ calcd for $\mathrm{C}_{24} \mathrm{H}_{33} \mathrm{OSi}\left(\mathrm{M}-\mathrm{CH}_{3}\right)^{+}$ 365.2301, found 365.2301. Major diastereomer: ${ }^{1} \mathrm{H} \mathrm{NMR}\left(500 \mathrm{MHz}, \mathrm{CDCl}_{3}\right) \delta 7.44(\mathrm{~m}$, $1 \mathrm{H}), 7.26(\mathrm{~m}, 9 \mathrm{H}), 3.12(\mathrm{dd}, J=13.9,4.1,1 \mathrm{H}), 2.82(\mathrm{dd}, J=13.9,11.7,1 \mathrm{H}), 2.63$ (dd, $J$ $=13.4,6.9,1 \mathrm{H}), 1.89(\mathrm{~m}, 2 \mathrm{H}), 1.54(\mathrm{~s}, 3 \mathrm{H}), 1.22(\mathrm{~s}, 9 \mathrm{H}), 0.81(\mathrm{~s}, 9 \mathrm{H}) ;{ }^{13} \mathrm{C}$ NMR $(125$ $\left.\mathrm{MHz}_{\mathrm{CDCl}}\right) \delta 151.0,143.2,128.4,128.3,127.8,126.3,125.8,125.5,81.4,45.2,36.4$, 34.3, 28.8, 28.6, 25.9, 25.7, 20.4. Minor diastereomer: ${ }^{1} \mathrm{H} \mathrm{NMR}\left(500 \mathrm{MHz}, \mathrm{CDCl}_{3}\right) \delta$ $7.44(\mathrm{~m}, 1 \mathrm{H}), 7.26(\mathrm{~m}, 9 \mathrm{H}), 3.17(\mathrm{dd}, J=13.6,2.9,1 \mathrm{H}), 2.74(\mathrm{~m}, 1 \mathrm{H}), 2.33(\mathrm{~m}, 1 \mathrm{H}), 1.84$ $(\mathrm{m}, 1 \mathrm{H}), 1.70(\mathrm{~m}, 1 \mathrm{H}), 1.41(\mathrm{~s}, 3 \mathrm{H}), 1.20(\mathrm{~s}, 9 \mathrm{H}), 1.10(\mathrm{~s}, 9 \mathrm{H}) ;{ }^{13} \mathrm{C}$ NMR $(125 \mathrm{MHz}$, $\left.\mathrm{CDCl}_{3}\right) \delta 147.9,143.0,128.4,128.2,127.7,127.6,125.9,124.3,81.6,42.2,36.2,32.3$, 26.7, 28.3, 25.7, 22.0, 21.9.

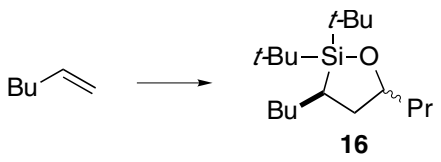

Oxasilacyclopentane (16). To a suspension of silver triflate $\left(0.0002 \mathrm{~g}, 7 \times 10^{-4} \mathrm{mmol}\right)$ in $\mathrm{CH}_{2} \mathrm{Cl}_{2}(4 \mathrm{~mL})$, inside the glove box, was added 1-hexene $(0.020 \mathrm{ml}, 0.161 \mathrm{mmol})$. A solution of $1(0.016 \mathrm{~g}, 0.071 \mathrm{mmol})$ in $\mathrm{CH}_{2} \mathrm{Cl}_{2}(1 \mathrm{~mL})$ was then added and the reaction mixture was stirred at room temperature for two hours. The reaction mixture was then filtered through an HPLC filter, and the filtrate was cooled to $-20{ }^{\circ} \mathrm{C}$. Butyraldehyde was then added $(0.02 \mathrm{ml}, 0.22 \mathrm{mmol})$ followed by zinc bromide $(0.005 \mathrm{~g}, 0.022 \mathrm{mmol})$, and the mixture was left at $-20{ }^{\circ} \mathrm{C}$ for $6 \mathrm{~h}$, and then rapidly warmed to room temperature. The mixture was stirred for an additional $4 \mathrm{~h}$ and then taken outside of the glove box. The mixture was diluted with $\mathrm{CH}_{2} \mathrm{Cl}_{2}(5 \mathrm{~mL})$ and washed with brine $(5 \mathrm{~mL})$. The water layer was extracted with $\mathrm{CH}_{2} \mathrm{Cl}_{2}(5 \mathrm{~mL})$. The combined organic phases were filtered through a cotton plug and concentrated to afford the product as a 39:61 mixture of diastereomers as determined by GC analysis. Purification by flash chromatography (pentane) afforded the product as a colorless oil $(0.017 \mathrm{~g}, 79 \%)$. Major diastereomer: ${ }^{1} \mathrm{H}$ $\operatorname{NMR}\left(500 \mathrm{MHz}, \mathrm{CDCl}_{3}\right) \delta 4.11(\mathrm{qd}, J=6.9,2.1,1 \mathrm{H}), 1.85(\mathrm{ddd}, J=12.8,8.5,2.4,1 \mathrm{H})$, 1.67 (ddd, $J=12.8,11.8,7.4,1 \mathrm{H}), 1.67(\mathrm{~m}, 1 \mathrm{H}), 1.48(\mathrm{~m}, 2 \mathrm{H}), 1.72(\mathrm{~m}, 8 \mathrm{H}), 1.04(\mathrm{~s}$, $9 \mathrm{H}), 1.03(\mathrm{~s}, 9 \mathrm{H}), 0.91(\mathrm{t}, J=7.2,3 \mathrm{H}), 0.90(\mathrm{t}, J=7.1,3 \mathrm{H}) ;{ }^{13} \mathrm{C}$ NMR $(125 \mathrm{MHz}$, $\left.\mathrm{CDCl}_{3}\right) \delta 76.7,40.5,37.6,33.6,30.2,28.6,28.4,22.8,22.1,21.9,19.9,19.5,14.2,14.1$; IR (thin film) 2958, 2929, 2857, 1468, 1014, $822 \mathrm{~cm}^{-1}$; HRMS (CI/isobutene) $\mathrm{m} / \mathrm{z}$ calcd 
for $\mathrm{C}_{18} \mathrm{H}_{37} \mathrm{OSi}(\mathrm{M}-\mathrm{H})^{+}$297.2613, found 297.2616. Minor diastereomer: ${ }^{1} \mathrm{H}$ NMR (400 $\left.\mathrm{MHz}, \mathrm{CDCl}_{3}\right) \delta 3.76(\mathrm{~m}, 1 \mathrm{H}), 2.13(\mathrm{ddd}, J=12.1,7.2,4.1,1 \mathrm{H}), 1.67(\mathrm{~m}, 2 \mathrm{H}), 1.40(\mathrm{~m}$, $8 \mathrm{H}), 1.14(\mathrm{~m}, 2 \mathrm{H}), 1.03(\mathrm{~s}, 9 \mathrm{H}), 1.02(\mathrm{~s}, 9 \mathrm{H}), 0.92(\mathrm{t}, J=7.2,3 \mathrm{H}), 0.90(\mathrm{t}, J=7.0,3 \mathrm{H})$; ${ }^{13} \mathrm{C}$ NMR $\left(125 \mathrm{MHz}, \mathrm{CDCl}_{3}\right) \delta 77.6,40.1,39.8,33.6,30.1,28.2,28.1,25.0,22.7,21.3$, 21.1, 19.0, 14.3, 14.1; IR (thin film) 2930, 2857, 1470, 1386, 1384, $1014 \mathrm{~cm}^{-1}$; HRMS (CI/isobutene) $m / z$ calcd for $\mathrm{C}_{18} \mathrm{H}_{37} \mathrm{OSi}(\mathrm{M}-\mathrm{H})^{+}$297.2613, found 297.2618. Anal. Calcd for $\mathrm{C}_{18} \mathrm{H}_{38} \mathrm{OSi}$ : C, 72.41; H, 12.83. Found C, 72.21; H, 12.97.

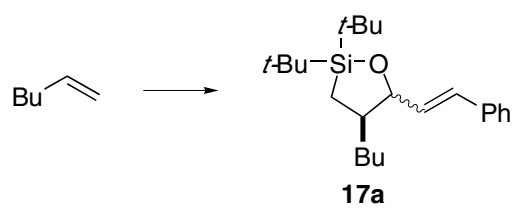

Oxasilacyclopentane (17a). To a cooled $\left(-20{ }^{\circ} \mathrm{C}\right)$ suspension of $\mathrm{AgOC}(\mathrm{O}) \mathrm{CF}_{3}(0.0002 \mathrm{~g}$, $\left.9 \times 10^{-4} \mathrm{mmol}\right)$ in $\mathrm{CH}_{2} \mathrm{Cl}_{2}(4 \mathrm{~mL})$, inside the glove box, was added 1-hexene $(0.020 \mathrm{ml}$, $0.160 \mathrm{mmol})$. A solution of cyclohexene silacyclopropane $1(0.025 \mathrm{~g}, 0.111 \mathrm{mmol})$ in $\mathrm{CH}_{2} \mathrm{Cl}_{2}$ (2 mL) was then added and the reaction mixture was stirred for $2 \mathrm{~h}$. Cinnamaldehyde $(0.030 \mathrm{ml}, 0.229 \mathrm{mmol})$ was then added followed by $\mathrm{CuI}(0.006 \mathrm{~g}$, $0.035 \mathrm{mmol})$. The reaction mixture was left at $-20{ }^{\circ} \mathrm{C}$ for $6 \mathrm{~h}$, and then rapidly warmed up to room temperature. The mixture was then stirred for an additional $4 \mathrm{~h}$ and then was taken outside of the glove box. The mixture was then diluted with $\mathrm{CH}_{2} \mathrm{Cl}_{2}(5 \mathrm{~mL})$ and washed with brine $(5 \mathrm{~mL})$. The water layer was then extracted with $\mathrm{CH}_{2} \mathrm{Cl}_{2}(5 \mathrm{~mL})$. The combined organic extracts were filtered through a cotton plug and concentrated to afford the product as a 96:4 mixture of regioisomers and an 82:18 mixture of diastereomers (as determined by GC analysis). Purification by flash chromatography (pentane:EtOAc = 99:1) afforded the product as a colorless oil $(0.034 \mathrm{~g}, 85 \%):{ }^{1} \mathrm{H} \mathrm{NMR}\left(400 \mathrm{MHz}, \mathrm{CDCl}_{3}\right)$ $\delta 7.41(\mathrm{~m}, 2 \mathrm{H}), 7.32(\mathrm{~m}, 2 \mathrm{H}), 7.22(\mathrm{~m}, 1 \mathrm{H}), 6.61(\mathrm{~d}, J=15.8,1 \mathrm{H}), 6.19,(\mathrm{dd}, J=15.8$, 7.2, 1H), $3.97(\mathrm{dd}, J=9.7,7.2,1 \mathrm{H}), 1.69(\mathrm{~m}, 1 \mathrm{H}), 1.58(\mathrm{~m}, 1 \mathrm{H}) .1 .46(\mathrm{~m}, 2 \mathrm{H}), 1.29(\mathrm{~m}$, 4H), $1.07(\mathrm{~s}, 9 \mathrm{H}), 1.06(\mathrm{~s}, 9 \mathrm{H}), 0.89(\mathrm{t}, J=7.2,3 \mathrm{H}), 0.47(\mathrm{dd}, J=14.7,11.8,1 \mathrm{H}) ;{ }^{13} \mathrm{C}$ NMR $\left(125 \mathrm{MHz}, \mathrm{CDCl}_{3}\right) \delta 137.1,131.8,130.8,128.4,127.3,126.5,85.2,45.4,33.8$, $30.2,27.7,27.6,22.8,20.6,19.9,14.1,13.0 ;$ IR (thin film) 3082, 3027, 2928, 1473, 1364, $991 \mathrm{~cm}^{-1}$; HRMS (CI) $m / z$ calcd for $\mathrm{C}_{23} \mathrm{H}_{38} \mathrm{OSi}(\mathrm{M})^{+} 358.2692$, found 358.2690.

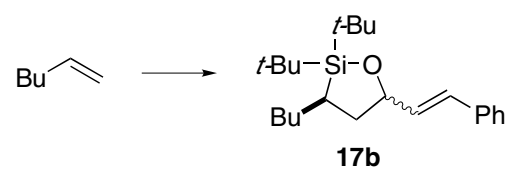

Oxasilacyclopentane (17b). The procedure for 17a was followed using $0.018 \mathrm{~g}$ of cyclohexene silacyclopropane $1(0.080 \mathrm{mmol}), 0.020 \mathrm{~mL}$ of 1 -hexene $(0.161 \mathrm{mmol})$, $0.0001 \mathrm{~g}$ of $\mathrm{AgOC}(\mathrm{O}) \mathrm{CF}_{3}\left(4 \times 10^{-4} \mathrm{mmol}\right), 0.020 \mathrm{~mL}$ of cinnamaldehyde $(0.153 \mathrm{mmol})$, and $0.005 \mathrm{~g}$ of $\mathrm{ZnI}_{2}(0.015 \mathrm{mmol})$ to give product $\mathbf{1 7 b}$ as a $73: 27$ mixture of diastereomers. Purification by flash chromatography (pentane:EtOAc $=99: 1$ ) yielded the product as a colorless oil (0.027 g, 92\%): IR (thin film) 3060, 3026, 2857, 1473, 1364, $963 \mathrm{~cm}^{-1}$; HRMS (CI/isobutane) $\mathrm{m} / \mathrm{z}$ calcd for $\mathrm{C}_{23} \mathrm{H}_{38} \mathrm{OSi}(\mathrm{M})^{+}$358.2692, found 358.2688. Selected data for major diastereomer: ${ }^{1} \mathrm{H}$ NMR $\left(400 \mathrm{MHz}, \mathrm{CDCl}_{3}\right) \delta 7.32(\mathrm{~m}$, $5 \mathrm{H}), 6.59(\mathrm{~d}, J=15.8,1 \mathrm{H}), 6.28(\mathrm{dd}, J=15.8,6.6,1 \mathrm{H}), 4.84(\mathrm{td}, J=6.6,1.9,1 \mathrm{H}), 2.12$ (ddd, $J=13.1,8.4,3.0,1 \mathrm{H}), 2.03(\mathrm{ddd}, J=13.1,11.3,7.6,1 \mathrm{H}), 1.76(\mathrm{~m}, 2 \mathrm{H}), 1.59(\mathrm{~m}$, 
$2 \mathrm{H}), 1.14(\mathrm{~s}, 9 \mathrm{H}), 1.12(\mathrm{~s}, 9 \mathrm{H}), 0.92,(\mathrm{t}, J=7.0,3 \mathrm{H}) ;{ }^{13} \mathrm{C} \mathrm{NMR}\left(100 \mathrm{MHz}, \mathrm{CDCl}_{3}\right) \delta$ 137.1, 133.1, 129.2, 128.5, 127.3, 126.4, 78.4, 40.6, 38.4, 33.6, 30.1, 28.7, 28.6, 22.8, 22.4, 20.1, 14.1. Selected data for minor diastereomer: ${ }^{1} \mathrm{H}$ NMR $\left(400 \mathrm{MHz}, \mathrm{CDCl}_{3}\right) \delta$ $7.32(\mathrm{~m}, 5 \mathrm{H}), 6.66(\mathrm{~d}, J=15.8,1 \mathrm{H}), 6.29(\mathrm{dd}, J=15.8,6.2,1 \mathrm{H}), 4.46(\mathrm{~m}, 1 \mathrm{H}), 2.27(\mathrm{~m}$, $1 \mathrm{H}), 1.13(\mathrm{~s}, 9 \mathrm{H}), 1.11(\mathrm{~s}, 9 \mathrm{H}), 0.95(\mathrm{t}, J=6.9,3 \mathrm{H}) ;{ }^{13} \mathrm{C} \mathrm{NMR}\left(100 \mathrm{MHz}, \mathrm{CDCl}_{3}\right) \delta$ 132.4, 129.0, 128.4, 127.2, 126.4, 77.4, 33.4, 29.9, 28.2, 25.1, 22.7, 22.2, 21.4, 21.1.

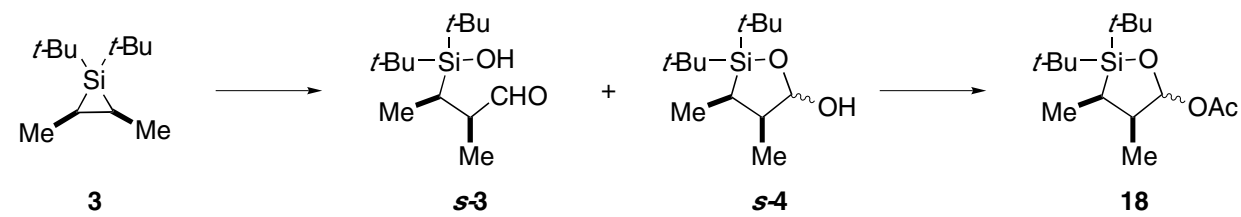

Acetate (18). To a cooled $\left(-20^{\circ} \mathrm{C}\right)$ solution of $\mathbf{3}(0.038 \mathrm{~g}, 0.192 \mathrm{mmol})$ in $\mathrm{CH}_{2} \mathrm{Cl}_{2}(4$ $\mathrm{mL})$, inside the glove box, was added a cold $\left(-20^{\circ} \mathrm{C}\right)$ solution of $N$-benzyl- $N$ methylformamide $(0.051 \mathrm{~g}, 0.342 \mathrm{mmol})$ followed by $\mathrm{CuI}(0.014 \mathrm{~g}, 0.074 \mathrm{mmol})$ and the mixture was left at $-20^{\circ} \mathrm{C}$ for six hours. The mixture was then rapidly warmed to room temperature and stirred for an additional $2 \mathrm{~h}$ after which it was taken outside the glove box. The reagents were then quenched with 2:1 mixture of $\mathrm{MeOH}_{\mathrm{Et}} \mathrm{H}_{3} \mathrm{~N}(4 \mathrm{~mL})$ and the mixture was concentrated. The residue was diluted with hexanes: $\mathrm{Et}_{3} \mathrm{~N}=95: 5(15 \mathrm{~mL})$ and washed with water once. Water layer was extracted with hexanes: $\mathrm{Et}_{3} \mathrm{~N}=95: 5$ mixture $(2 \times 10 \mathrm{~mL})$. The combined organic extracts were filtered through a cotton plug and concentrated. The residue was dissolved in $5 \mathrm{~mL}$ of THF and cooled to $0{ }^{\circ} \mathrm{C}$. The solution of $\mathrm{CuSO}_{4}(2.50 \mathrm{~mL}$ of $0.1 \mathrm{M}$ solution, $0.25 \mathrm{mmol})$ was added dropwise and the mixture was allowed to reach room temperature overnight. The reaction mixture was then diluted with MTBE $(15 \mathrm{~mL})$ and washed with water once $(10 \mathrm{~mL})$. The water layer was extracted with $\operatorname{MTBE}(3 \times 10 \mathrm{~mL})$. The combined organic extracts were concentrated in vacuo and the resultant oil was redissolved in $\mathrm{CH}_{2} \mathrm{Cl}_{2}$, filtered through cotton plug, and concentrated. The resulting mixture of $\boldsymbol{s - 3}$ and $\boldsymbol{s} \mathbf{- 4}$ was dissolved in $\mathrm{CH}_{2} \mathrm{Cl}_{2}(10 \mathrm{~mL}), \mathrm{Et}_{3} \mathrm{~N}(0.57 \mathrm{~mL}, 4.14 \mathrm{mmol})$ was added, followed by acetic anhydride $(0.18 \mathrm{~mL}, 1.91 \mathrm{mmol})$, and a few crystals of DMAP. The reaction mixture was left stirring at ambient temperature overnight and then concentrated in vacuo. Purification by flash chromatography (pentane:EtOAc $=100: 0-98: 2$ ) afforded 18 as a colorless oil ( $0.049 \mathrm{~g}, 90 \%$ over three steps). The characterization data match the previously reported spectra of this compound. ${ }^{4}$

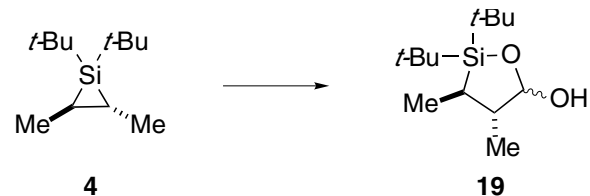

Oxasilacyclopentane hemiacetal (19). To a cooled $\left(-20^{\circ} \mathrm{C}\right)$ solution of $4(0.053 \mathrm{~g}$, $0.267 \mathrm{mmol})$ in $\mathrm{CH}_{2} \mathrm{Cl}_{2}(3 \mathrm{~mL})$, inside the glove box, was added a cold $\left(-20^{\circ} \mathrm{C}\right)$ solution of $N$-benzyl- $N$-methylformamide $(0.056 \mathrm{~g}, 0.375 \mathrm{mmol})$ followed by $\mathrm{CuI}(0.016 \mathrm{~g}, 0.084$ $\mathrm{mmol}$ ) and the mixture was left at $-20{ }^{\circ} \mathrm{C}$ for six hours. The mixture was then rapidly warmed to room temperature and stirred for an additional $2 \mathrm{~h}$ and then taken outside the glove box. The reagents were quenched with 2:1 mixture of $\mathrm{MeOH}_{\mathrm{Et}} \mathrm{N}(4 \mathrm{~mL})$ and the mixture was concentrated. The residue was diluted with hexanes: $\mathrm{Et}_{3} \mathrm{~N}=95: 5(15 \mathrm{~mL})$ 
and washed with water once. Water layer was extracted with hexanes: $\mathrm{Et}_{3} \mathrm{~N}=$ 95:5 mixture $(2 \times 10 \mathrm{~mL})$. The combined organic extracts were filtered through a cotton plug and concentrated. The residue was dissolved in $5 \mathrm{~mL}$ of THF and cooled to $0{ }^{\circ} \mathrm{C}$. The solution of $\mathrm{CuSO}_{4}(2.50 \mathrm{~mL}$ of $0.1 \mathrm{M}$ solution, $0.25 \mathrm{mmol})$ was added dropwise and the mixture was allowed to reach room temperature overnight. The reaction mixture was then diluted with MTBE $(15 \mathrm{~mL})$ and washed with water once $(10 \mathrm{~mL})$. The water layer was extracted with MTBE (3 x $10 \mathrm{~mL})$. The combined organic extracts were concentrated in vacuo and the resultant oil was redissolved in $\mathrm{CH}_{2} \mathrm{Cl}_{2}$, filtered through cotton plug, and concentrated. Purification by flash chromatography (pentane:EtOAc = 95:5 - 90:10) yielded the resultant hemiacetal as a 79:21 mixture of epimers, as determined by ${ }^{1} \mathrm{H}$ NMR spectroscopy $(0.059 \mathrm{~g}, 91 \%$ ): IR (thin film) 2929, 1123, 1050, 1019, 991, $903 \mathrm{~cm}^{-1}$; HRMS (CI/isobutene) $\mathrm{m} / \mathrm{z}$ calcd for $\mathrm{C}_{13} \mathrm{H}_{28} \mathrm{O}_{2} \mathrm{Si}(\mathrm{M})^{+}$244.1858, found 244.1860. Anal. Calcd for $\mathrm{C}_{13} \mathrm{H}_{28} \mathrm{O}_{2} \mathrm{Si}$ : C, 63.87; $\mathrm{H}, 11.55$. Found $\mathrm{C}, 63.93 ; \mathrm{H}$, 11.57. Major epimer: ${ }^{1} \mathrm{H} \mathrm{NMR}\left(500 \mathrm{MHz}, \mathrm{CDCl}_{3}\right) \delta 4.79(\mathrm{dd}, J=7.5,5.1,1 \mathrm{H}), 3.83(\mathrm{~d}$, $J=5.1,1 \mathrm{H}), 1.48(\mathrm{dqd}, J=3.6,7.5,6.5,1 \mathrm{H}), 1.12(\mathrm{~d}, J=7.5,3 \mathrm{H}), 1.01(\mathrm{~s}, 9 \mathrm{H}), 0.99(\mathrm{~d}$, $J=6.5,3 \mathrm{H}), 0.95(\mathrm{~s}, 9 \mathrm{H}), 0.88(\mathrm{~m}, 1 \mathrm{H}) ;{ }^{13} \mathrm{C} \mathrm{NMR}\left(125 \mathrm{MHz}, \mathrm{CDCl}_{3}\right) \delta 102.9,48.1$, 27.83, 28.81, 23.8, 21.0, 20.7, 15.5, 12.8; Selected data for minor epimer: ${ }^{1} \mathrm{H}$ NMR (500 $\left.\mathrm{MHz}, \mathrm{CDCl}_{3}\right) \delta 5.34(\mathrm{~m}, 1 \mathrm{H}), 3.03(\mathrm{~d}, J=2.5,1 \mathrm{H}), 1.78(\mathrm{~m}, 1 \mathrm{H}), 1.00(\mathrm{~s}, 9 \mathrm{H}), 0.98(\mathrm{~s}$, $9 \mathrm{H}) ;{ }^{13} \mathrm{C} \mathrm{NMR}\left(125 \mathrm{MHz}, \mathrm{CDCl}_{3}\right) \delta 98.6,45.0,28.5,21.5,20.1,19.8,13.6,12.4$.

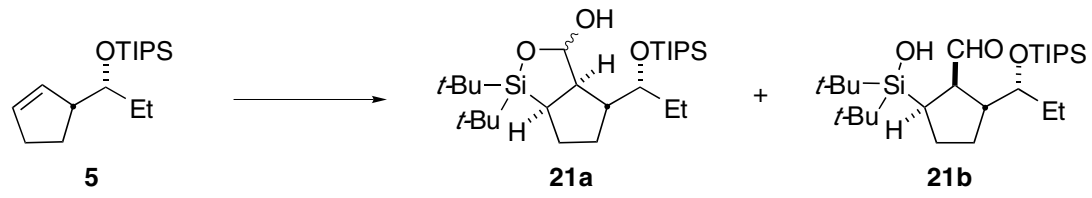

Hemiacetal (21a) and aldehyde (21b). To a suspension of $\mathrm{AgOC}(\mathrm{O}) \mathrm{CF}_{3}(0.0002 \mathrm{~g}, 8 \mathrm{x}$ $\left.10^{-4} \mathrm{mmol}\right)$ in toluene $(2 \mathrm{~mL})$, inside the glove box, was added $5(0.066 \mathrm{~g}, 0.242 \mathrm{mmol})$. A solution of $1(0.044 \mathrm{~g}, 0.196 \mathrm{mmol})$ in toluene $(1 \mathrm{~mL})$ was then added and the reaction mixture was stirred for $5 \mathrm{~h}$. The reaction mixture was then filtered through an HPLC filter and cooled to $-20^{\circ} \mathrm{C}$. A cooled $\left(-20^{\circ} \mathrm{C}\right)$ solution of $N$-benzyl- $N$-methyl formamide $(0.035 \mathrm{~g}, 0.23 \mathrm{mmol})$ was then added followed by $\mathrm{CuI}(0.009 \mathrm{mg}, 0.047 \mathrm{mmol})$, and the mixture was left at $-20{ }^{\circ} \mathrm{C}$ for $4 \mathrm{~h}$. The mixture was then rapidly warmed to room temperature and left stirring overnight. The reaction mixture was then taken outside of the glove box. The reagents were quenched with a 2:1 mixture of $\mathrm{MeOH}: \mathrm{Et}_{3} \mathrm{~N}(1 \mathrm{~mL})$ and the mixture was concentrated. The residue was diluted with hexanes: $\mathrm{Et}_{3} \mathrm{~N}=95: 5$ (10 $\mathrm{mL})$ and washed with water $(5 \mathrm{~mL})$. The water layer was extracted with hexanes: $\mathrm{Et}_{3} \mathrm{~N}=$ 95:5 mixture $(2 \times 10 \mathrm{~mL})$. The combined organic phases were filtered through a cotton plug and concentrated. The residue was dissolved in $10 \mathrm{~mL}$ of THF and cooled to $0{ }^{\circ} \mathrm{C}$. A solution of $\mathrm{CuSO}_{4}(1.50 \mathrm{~mL}$ of $0.1 \mathrm{M}$ solution, $0.15 \mathrm{mmol})$ was added dropwise and the mixture was allowed to reach room temperature overnight. The reaction mixture was then diluted with MTBE $(15 \mathrm{~mL})$ and washed with water $(10 \mathrm{~mL})$. The water layer was extracted with MTBE (3 x $10 \mathrm{~mL})$. The combined organic extracts were concentrated in vacuo and the resultant oil was redissolved in $\mathrm{CH}_{2} \mathrm{Cl}_{2}$, filtered through cotton plug, and concentrated. Purification by flash chromatography (pentane:EtOAc $=100: 0-96: 4$ ) yielded the products 21a (d.r. $=9: 91$ ) and $\mathbf{2 1 b}$ as a 83:17 mixture of compounds. The spectral data for these compounds match the already reported values. ${ }^{5}$ 


\section{Construction of 1,3-diols from oxasilacyclopentanes}

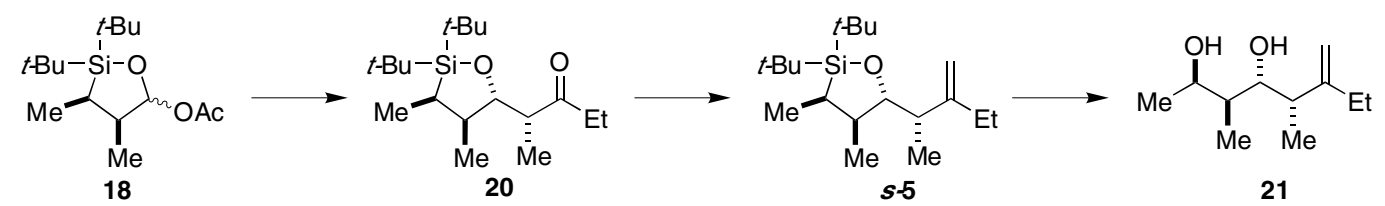

Diol (21). To a cooled $\left(-78{ }^{\circ} \mathrm{C}\right)$ solution of $\mathbf{1 8}(1.500 \mathrm{~g}, 5.236 \mathrm{mmol})$ in $\mathrm{CH}_{2} \mathrm{Cl}_{2}(58 \mathrm{~mL})$ was added $\mathrm{SnBr}_{4}\left(5.20 \mathrm{~mL}\right.$ of a $1.0 \mathrm{M}$ solution in $\left.\mathrm{CH}_{2} \mathrm{Cl}_{2}, 0.05 \mathrm{mmol}\right)$, followed by (Z)-1ethyl-1-trimethylsilyloxypropene $(4.930 \mathrm{~g}, 31.141 \mathrm{mmol})$. After stirring for $15 \mathrm{~min}$, the reaction mixture was poured into $150 \mathrm{~mL}$ of saturated aqueous $\mathrm{NaHCO}_{3}$ and diluted with $100 \mathrm{~mL}$ of $\mathrm{CH}_{2} \mathrm{Cl}_{2}$, and the layers were separated. The aqueous layer was extracted with $3 \times 50 \mathrm{~mL}$ of $\mathrm{CH}_{2} \mathrm{Cl}_{2}$. The combined organic extracts were washed with brine, filtered through a cotton plug, and concentrated in vacuo. Purification by flash chromatography (hexanes:EtOAc $=99: 1)$ afforded 20 as a colorless oil $(1.555 \mathrm{~g}, 95 \%)$, as a 90:9:1 mixture of diastereomers: ${ }^{1} \mathrm{H}$ NMR $\left(400 \mathrm{MHz}, \mathrm{CDCl}_{3}\right) \delta 3.87(\mathrm{dd}, J=10.4,2.8,1 \mathrm{H}), 2.57(\mathrm{q}, J$ $=7.2,2 \mathrm{H}), 2.50(\mathrm{dq}, J=7.0,2.8,1 \mathrm{H}), 2.03-1.97,(\mathrm{~m}, 1 \mathrm{H}), 1.42$ (quintet, $J=8.0,1 \mathrm{H})$, $1.08(\mathrm{~d}, J=7.0,3 \mathrm{H}), 1.10,(\mathrm{~d}, J=8.0,3 \mathrm{H}), 1.06(\mathrm{t}, J=7.2,3 \mathrm{H}), 1.05(\mathrm{~s}, 9 \mathrm{H}), 0.99(\mathrm{~s}$, $9 \mathrm{H}), 0.89(\mathrm{~d}, J=7.2,3 \mathrm{H}) ;{ }^{13} \mathrm{C} \mathrm{NMR}\left(125 \mathrm{MHz}, \mathrm{CDCl}_{3}\right) \delta 214.0,81.1,49.3,38.6,33.2$, 28.6, 28.1, 22.1, 20.6, 19.8, 12.4, 10.8, 8.4, 7.9; IR (thin film) 2967, 2861, 1710, 1468, $1384 \mathrm{~cm}^{-1}$; HRMS (CI/isobutane) $m / z$ calcd for $\mathrm{C}_{18} \mathrm{H}_{37} \mathrm{O}_{2} \mathrm{Si}(\mathrm{M}+\mathrm{H})^{+} 313.2563$, found 313.2568. Anal. Calcd for $\mathrm{C}_{18} \mathrm{H}_{36} \mathrm{O}_{2} \mathrm{Si}$ : C, 69.17; H, 11.61. Found C, 69.42; H, 11.70. The stereochemistry of the major isomer has been reported ${ }^{6}$

To a cooled $\left(0{ }^{\circ} \mathrm{C}\right)$ suspension of triphenylmethylphopsphonium bromide $(0.058$ $\mathrm{g}, 0.162 \mathrm{mmol})$ in THF $(0.38 \mathrm{~mL})$ was added $n$-BuLi $(0.064 \mathrm{~mL}$ of $2.2 \mathrm{M}$ solution in hexanes). After 15 minutes, the yellow mixture was cooled to $-78{ }^{\circ} \mathrm{C}$ and ketone 20 $(0.022 \mathrm{~g}, 0.070 \mathrm{mmol})$ was added as a solution in THF $(0.90 \mathrm{~mL})$. The solution was allowed to warm to room temperature and then heated to reflux for $6 \mathrm{~h}$. The mixture was cooled to room temperature, quenched with $0.10 \mathrm{~mL}$ of methanol, and suspended in 20 $\mathrm{mL}$ of pentane. The resulting suspension was filtered through silica gel and concentrated in vacuo. Purification by flash chromatography (hexanes) yielded the product $\boldsymbol{s} \mathbf{- 5}$ as a colorless oil (0.016 g, 73\%): ${ }^{1} \mathrm{H}$ NMR $\left(500 \mathrm{MHz}, \mathrm{CDCl}_{3}\right) \delta 4.93$ (bs, 1H), 4.74 (bs, 1H), $3.54(\mathrm{dd}, J=10.1,2.3,1 \mathrm{H}), 2.23(\mathrm{dq}, J=6.9,2.2,1 \mathrm{H}), 2.12(\mathrm{~m}, 2 \mathrm{H}), 1.97(\mathrm{dqd}, J=$ $10.1,8.2,7.0,1 \mathrm{H}), 1.40$ (quintet, $J=8.2,1 \mathrm{H}), 1.06(\mathrm{~s}, 9 \mathrm{H}$ and $\mathrm{m}, 3 \mathrm{H}), 1.04(\mathrm{t}, J=7.4$, $3 \mathrm{H}), 1.02(\mathrm{~d}, J=6.9,3 \mathrm{H}), 1.00(\mathrm{~s}, 9 \mathrm{H}), 0.88(\mathrm{~d}, J=7.0,3 \mathrm{H}) ;{ }^{13} \mathrm{C}$ NMR $(125 \mathrm{MHz}$, $\left.\mathrm{CDCl}_{3}\right) \delta 156.0,107.3,83.8,42.3,39.1,29.9,28.9,28.4,27.3,22.3,20.8,20.2,13.3$, 12.8, 11.2 IR (thin film): 3083, 2964, 1468, $1386 \mathrm{~cm}^{-1}$; HRMS (CI/isobutane) $\mathrm{m} / z$ calcd for $\mathrm{C}_{19} \mathrm{H}_{39} \mathrm{OSi}(\mathrm{M}+\mathrm{H})^{+} 311.2770$, found 311.2770. Anal. Calcd for $\mathrm{C}_{19} \mathrm{H}_{38} \mathrm{OSi}$ : C, 73.47; $\mathrm{H}, 12.33$. Found $\mathrm{C}, 73.33 ; \mathrm{H} 12.20$.

To a cooled $\left(0{ }^{\circ} \mathrm{C}\right)$ suspension of $\mathrm{KH}(0.017 \mathrm{~g}, 0.424 \mathrm{mmol})$ in $0.35 \mathrm{~mL}$ of NMP was added tert-butylhydroperoxide $(0.055 \mathrm{~mL}$ of $70 \%$ aqueous solution, $0.427 \mathrm{mmol})$ dropwise. After the solution was warmed to $25^{\circ} \mathrm{C}$, the solution of oxasilacyclopentane $s$ $5(0.044 \mathrm{~g}, 0.143 \mathrm{mmol})$ in $0.80 \mathrm{~mL}$ of NMP was added. After stirring for $5 \mathrm{~min}, \mathrm{CsF}$ $(0.221 \mathrm{~g}, 1.454 \mathrm{mmol})$ was added, followed by TBAF $(0.010 \mathrm{~mL}$ of $1.0 \mathrm{M}$ solution in THF, $0.010 \mathrm{mmol})$. The reaction mixture was heated at $40{ }^{\circ} \mathrm{C}$ for $6 \mathrm{~h}$. After the mixture was cooled to $25^{\circ} \mathrm{C}$, a solution of $\mathrm{Na}_{2} \mathrm{~S}_{2} \mathrm{O}_{3}(3.0 \mathrm{~mL}$ of $1.3 \mathrm{M}$ solution, $3.9 \mathrm{mmol})$ was 
added and the mixture was stirred for $1 \mathrm{~h}$. The mixture was then washed with $4 \times 10 \mathrm{~mL}$ of MTBE. The combined organic extracts were washed with $5 \times 10 \mathrm{~mL}$ water, dried over $\mathrm{Na}_{2} \mathrm{SO}_{4}$ and concentrated in vacuo. Purification by flash chromatography (hexanes:EtOAc $=91: 9-84: 16)$ afforded the product 21 as colorless oil $(0.024 \mathrm{~g}, 90 \%)$ : ${ }^{1} \mathrm{H}$ NMR $\left(500 \mathrm{MHz}, \mathrm{CDCl}_{3}\right) \delta 4.94(\mathrm{~m}, 1 \mathrm{H}), 4.84(\mathrm{bs}, 1 \mathrm{H}), 4.06$ (d quintet, $J=6.6,2.3$, $1 \mathrm{H}), 3.56(\mathrm{ddd}, J=7.8,4.4,2.3,1 \mathrm{H}), 3.05(\mathrm{~d}, J=6.7,1 \mathrm{H}), 2.37(\mathrm{dq}, J=6.9,4.4,1 \mathrm{H})$, $2.27(\mathrm{~d}, J=2.3,1 \mathrm{H}), 2.16-1.94(\mathrm{~m}, 2 \mathrm{H}), 1.80$ (d quintet, $J=7.2,2.3,1 \mathrm{H}), 1.18(\mathrm{~d}, J=$ $6.6,3 \mathrm{H}), 1.06(\mathrm{t}, J=7.4,3 \mathrm{H}), 1.05(\mathrm{~d}, J=6.9,3 \mathrm{H}), 0.88(\mathrm{~d}, J=7.1,3 \mathrm{H}) ;{ }^{13} \mathrm{C} \mathrm{NMR}(125$ $\left.\mathrm{MHz}, \mathrm{CDCl}_{3}\right) \delta 153.9,109.4,74.5,69.8,41.9,39.4,28.0,19.0,12.4,12.3,12.0$; IR (thin film) 3355, 3083, 2969, 1642, 1459, $1112 \mathrm{~cm}^{-1}$; HRMS (CI/isobutane) $\mathrm{m} / \mathrm{z}$ calcd for $\mathrm{C}_{11} \mathrm{H}_{21} \mathrm{O}_{2}(\mathrm{M}-\mathrm{H})^{+}$185.1541, found 185.1543. Anal. Cald for $\mathrm{C}_{11} \mathrm{H}_{22} \mathrm{O}_{2}$ : C, 70.92; $\mathrm{H}$, 11.90. Found C, 70.68; H, 12.08 .

\section{References}

(1) Pangborn, A. B.; Giardello, M. A.; Grubbs, R. H.; Rosen, R. K.; Timmers, F. J Organometallics 1996, 15, 1518-1520.

(2) Cirakovic, J.; Driver, T. G.; Woerpel, K. A. J. Am. Chem. Soc. 2002, 124, 93709371.

(3) Boudjouk, P.; Samaraweera, U.; Sooriyakumaran, R.; Chrusciel, J.; Anderson, K. R. Angew. Chem. Int. Ed. Engl. 1988, 27, 1355-1356.

(4) Nguyen, P. T.; Palmer, W. S.; Woerpel, K. A. J. Org. Chem. 1999, 64, 18431848.

(5) Driver, T. G.; Franz, A. K.; Woerpel, K. A. J. Am. Chem. Soc. 2002, 124, 65246525.

(6) Bear, T. J.; Shaw, J. T.; Woerpel, K. A. J. Org. Chem. 2002, 67, 2056-2064. 


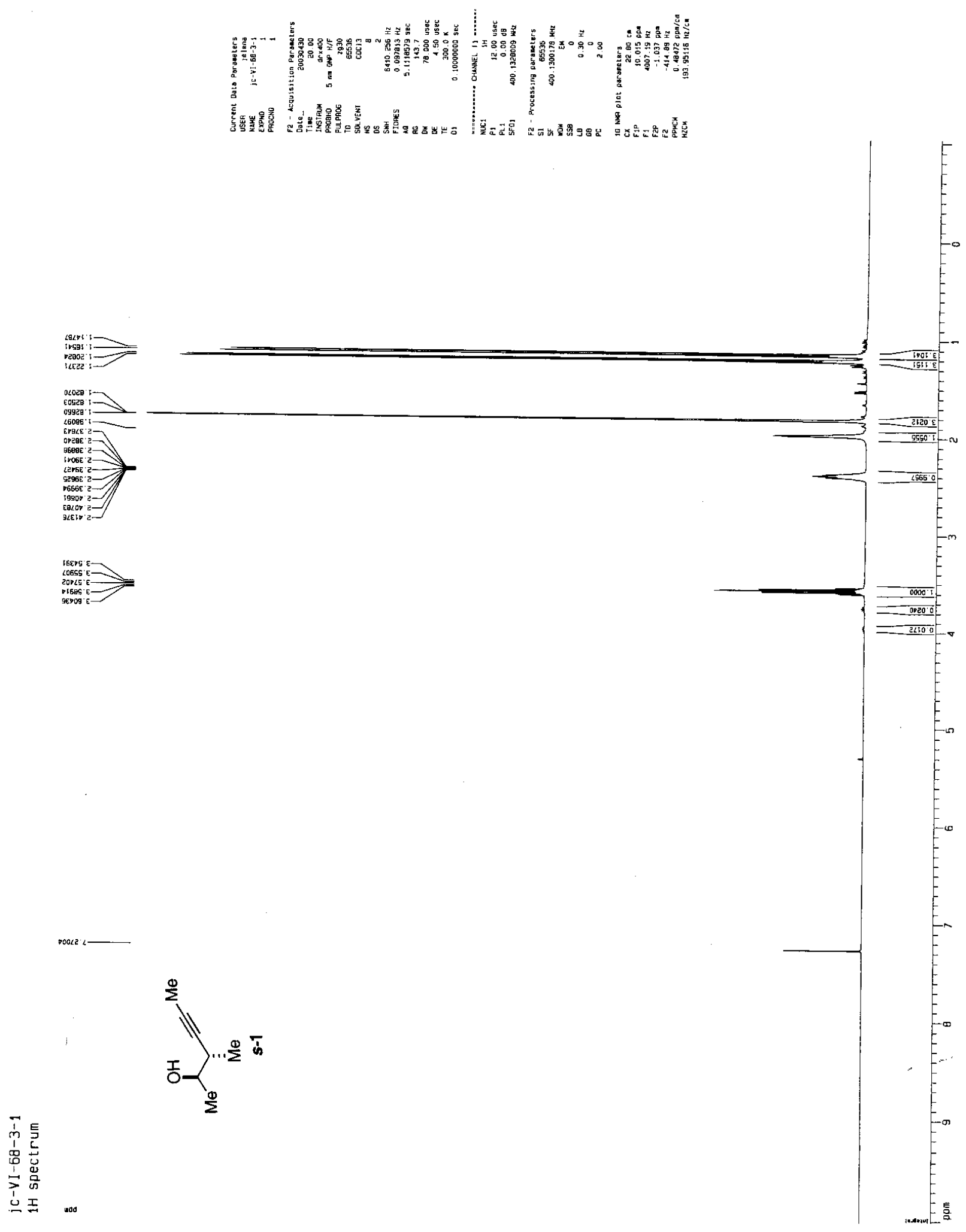




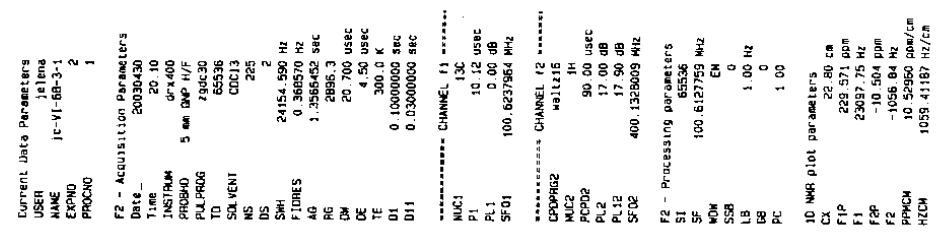

$205 \varepsilon$

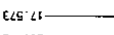

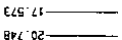

$\$ 16 \cdot \cdots$
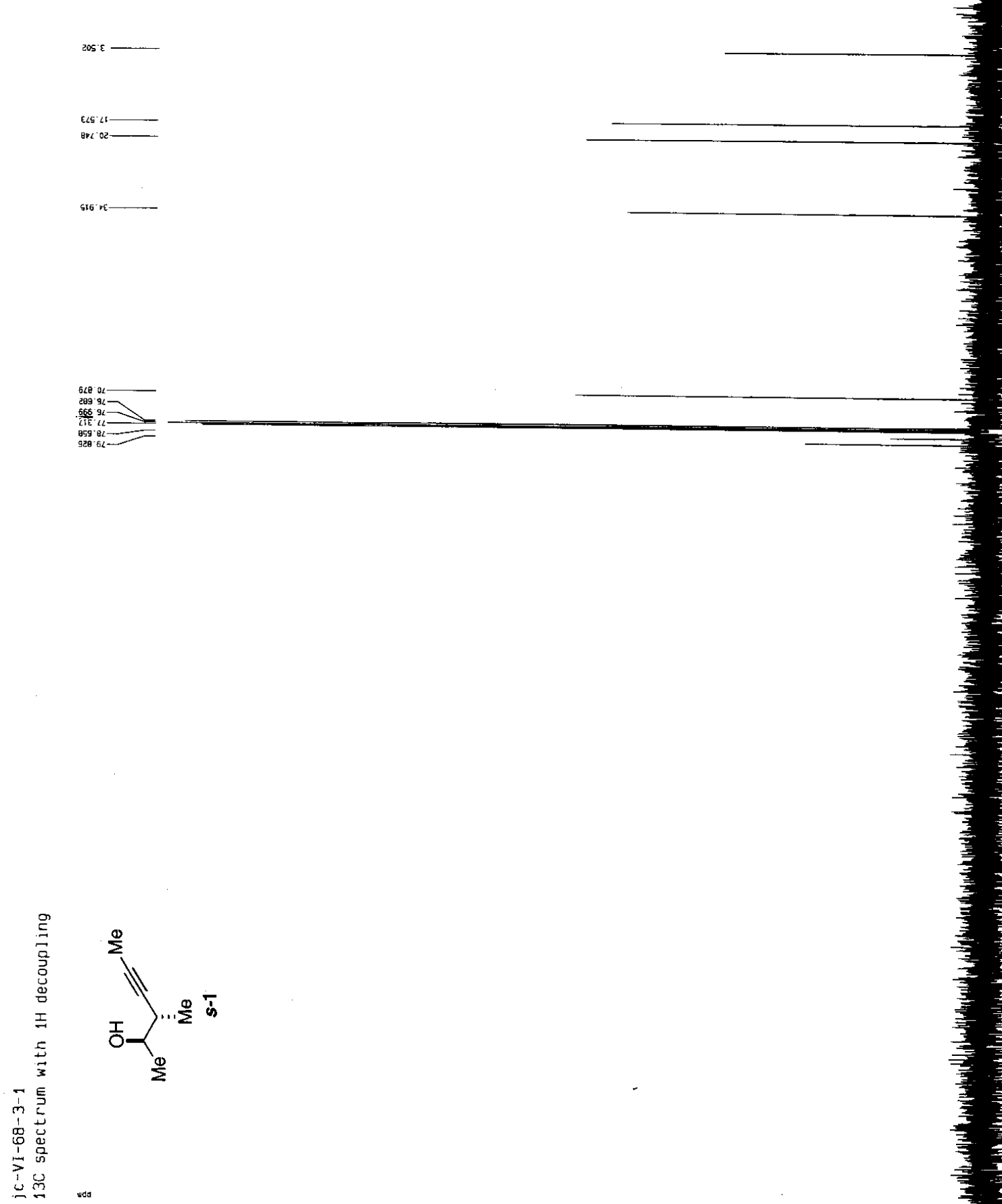


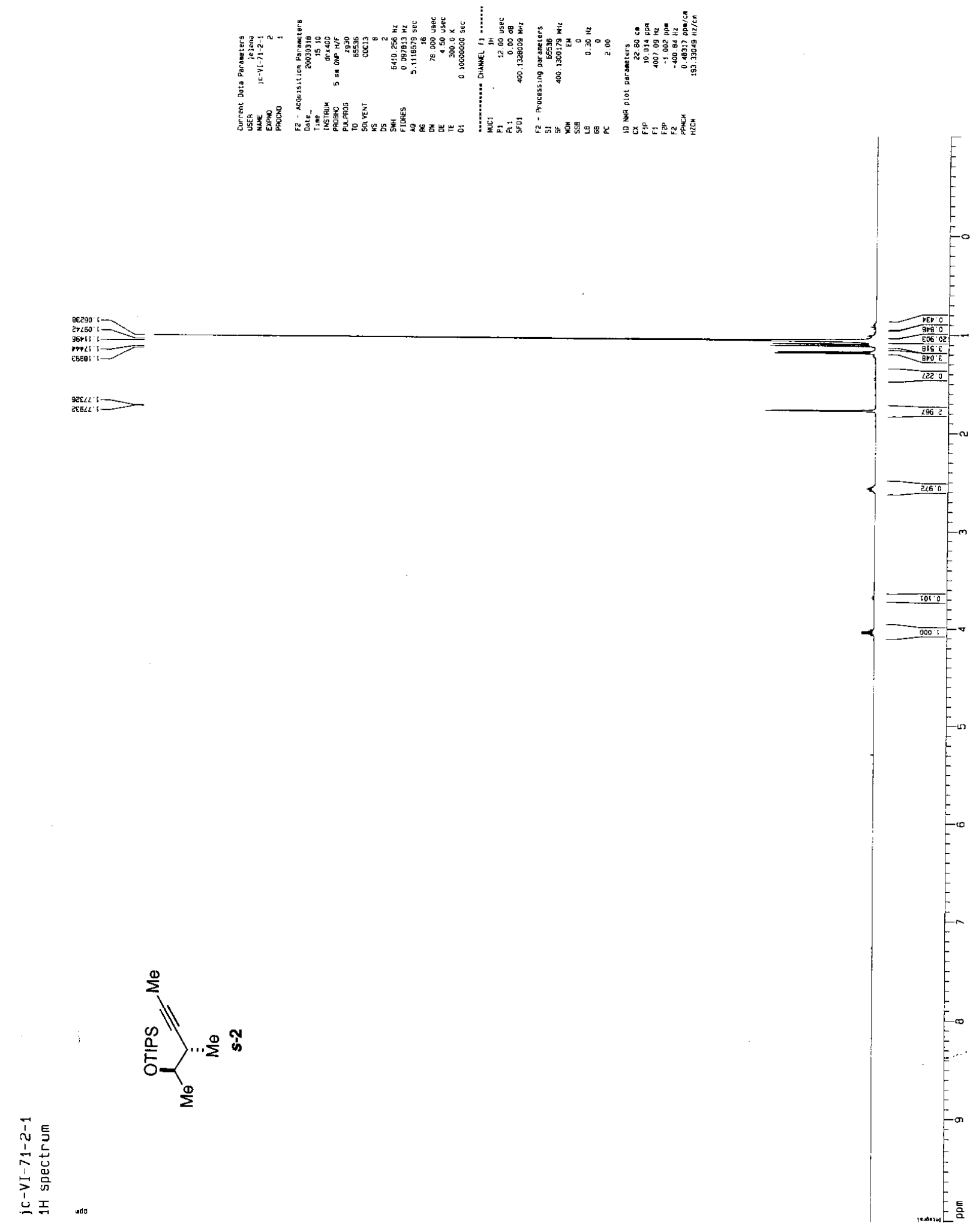



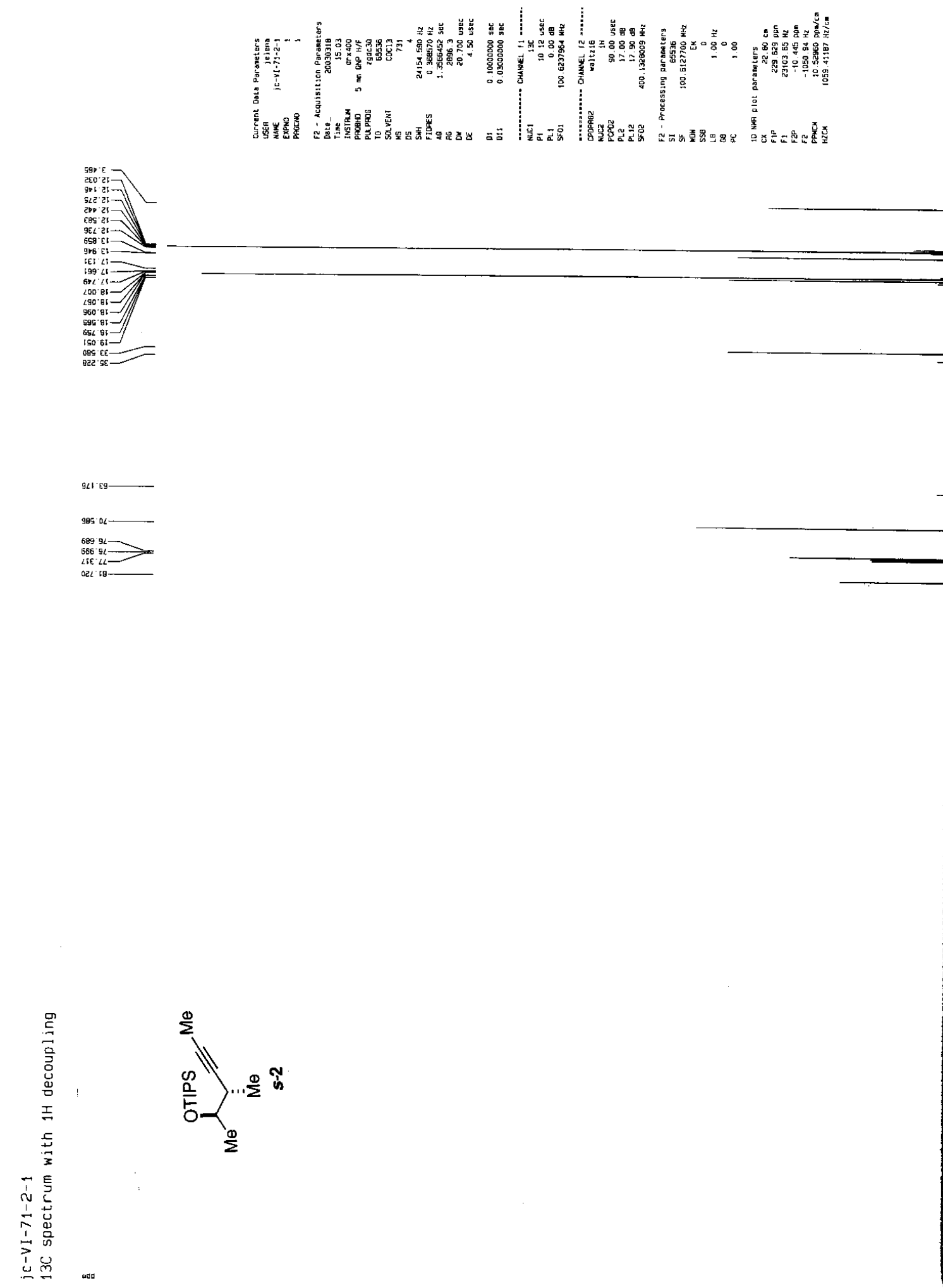


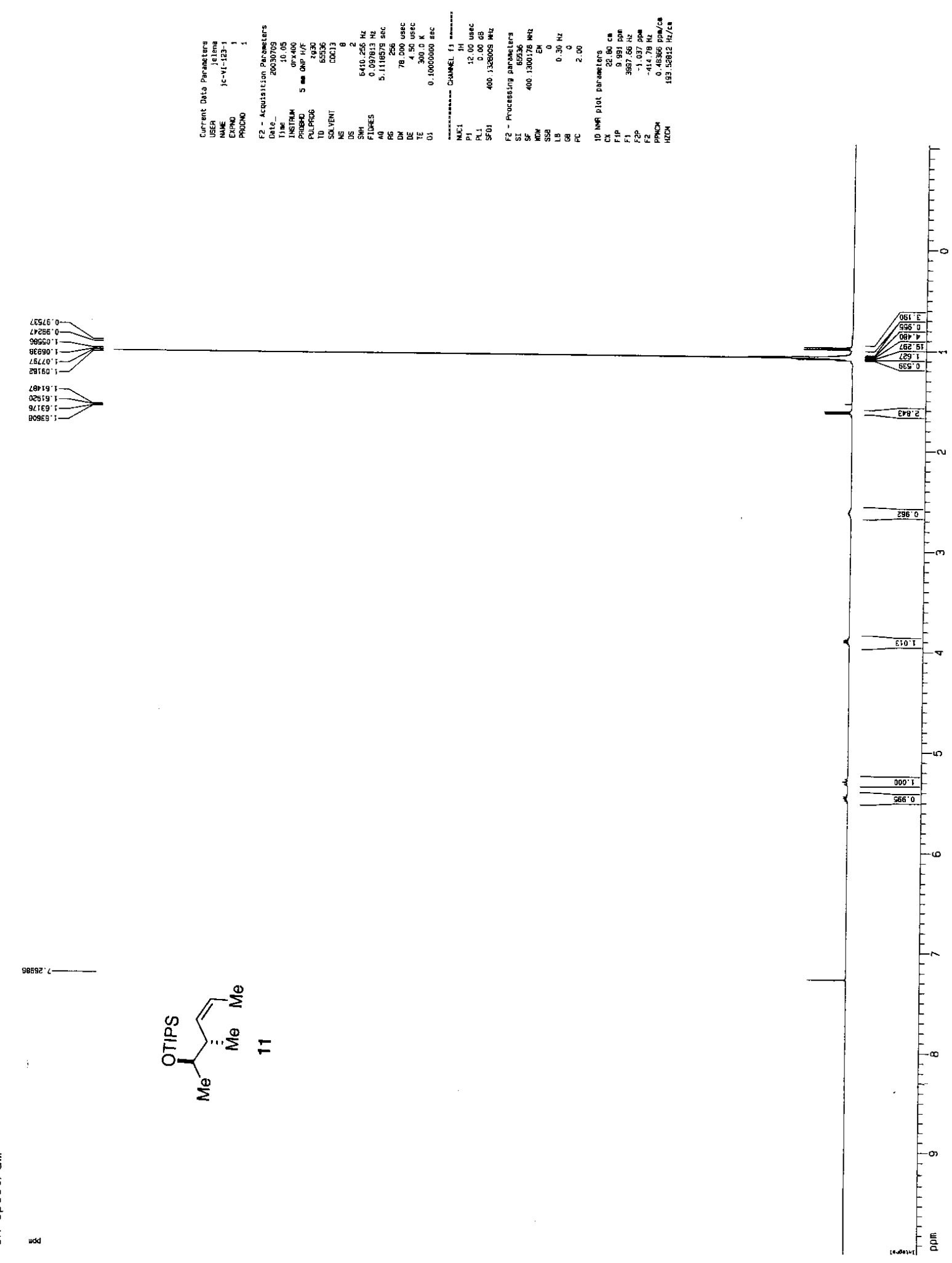




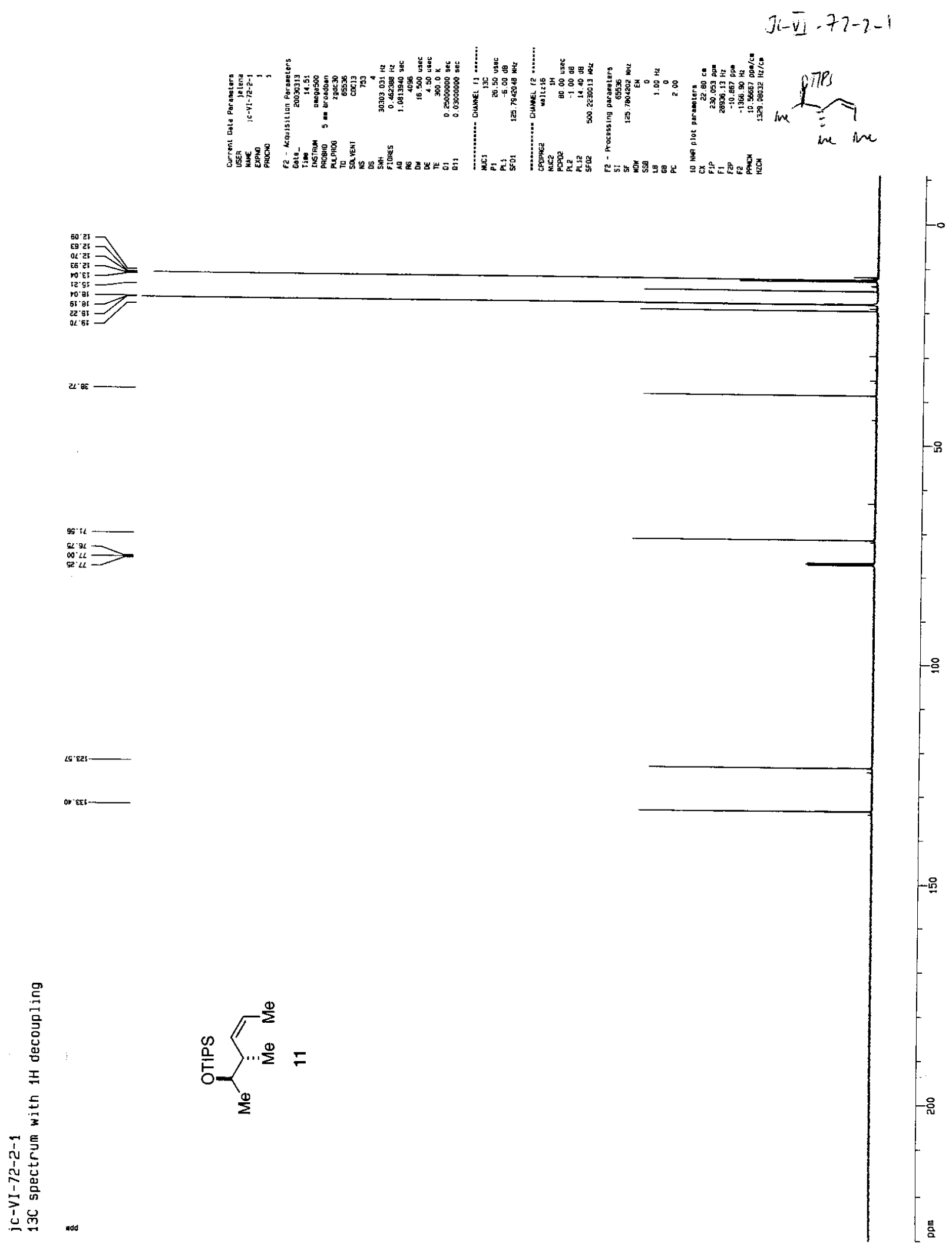



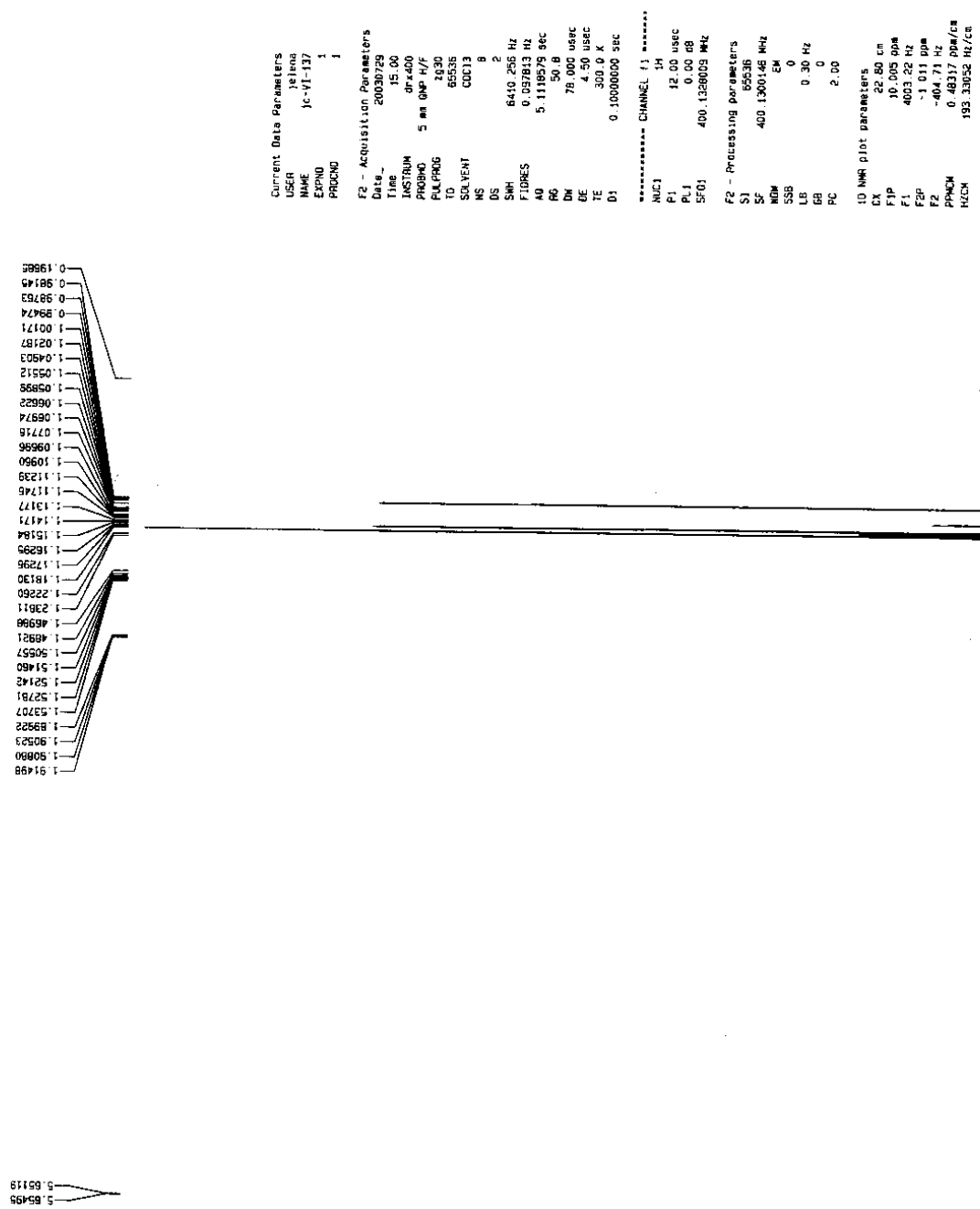

. 


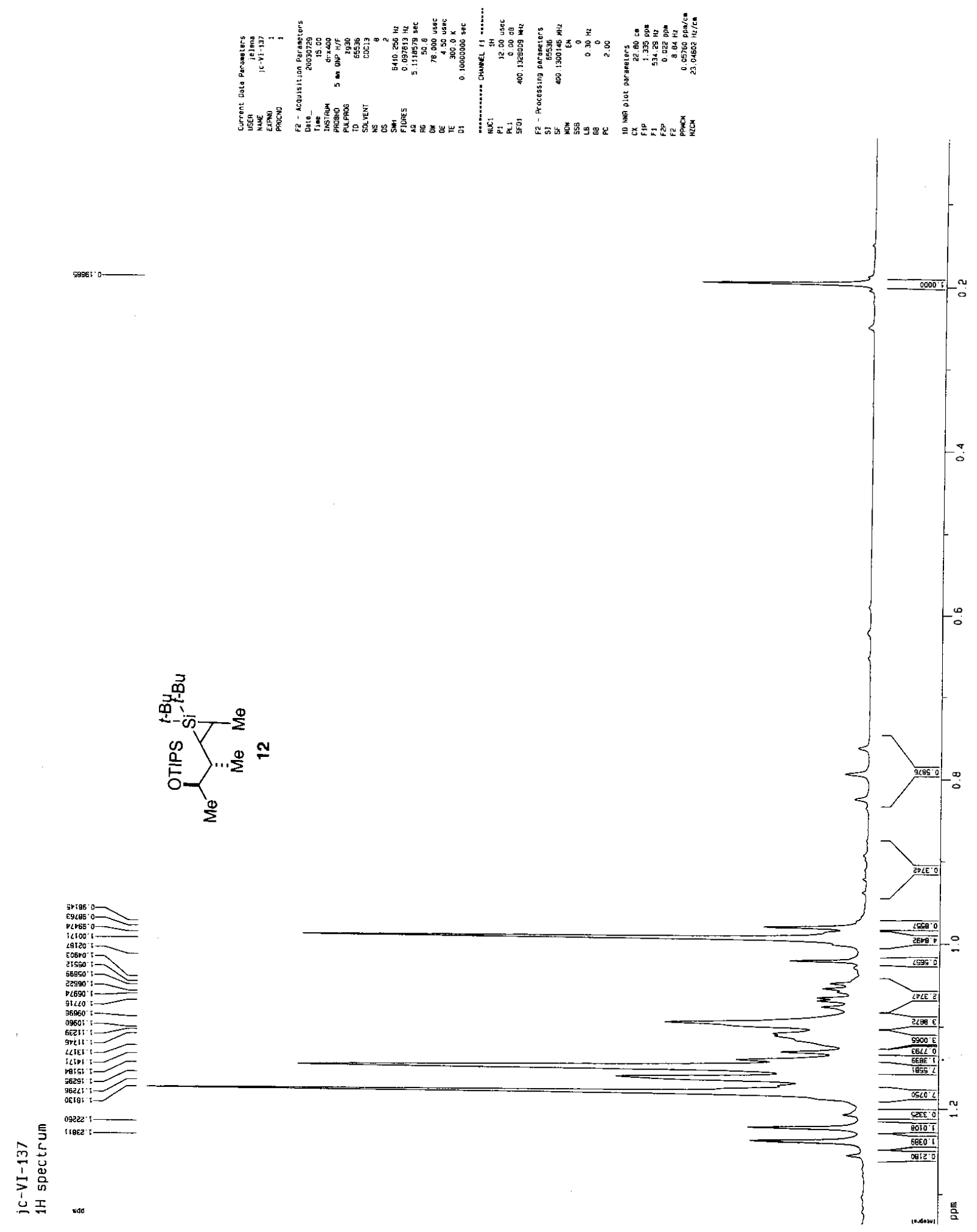




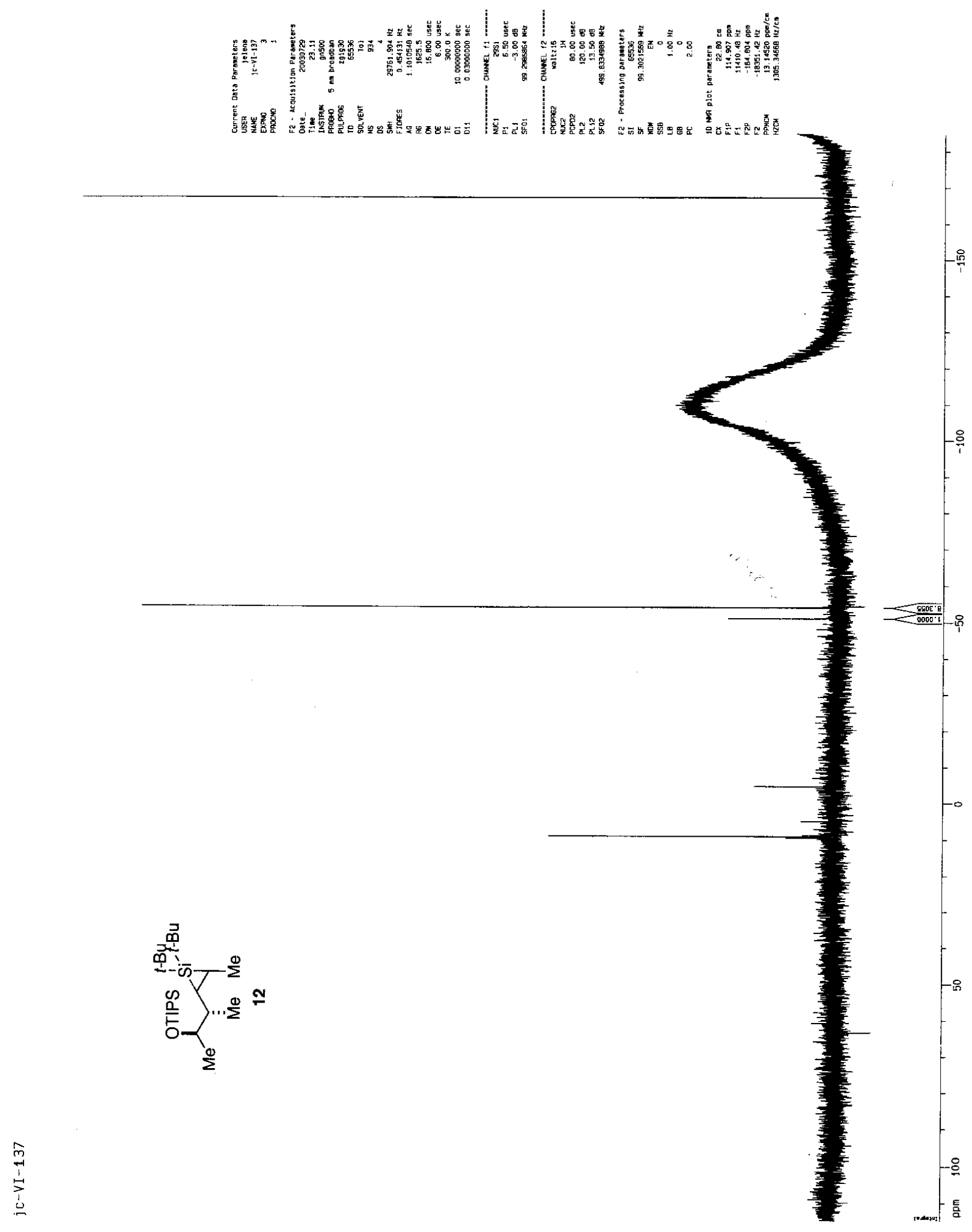



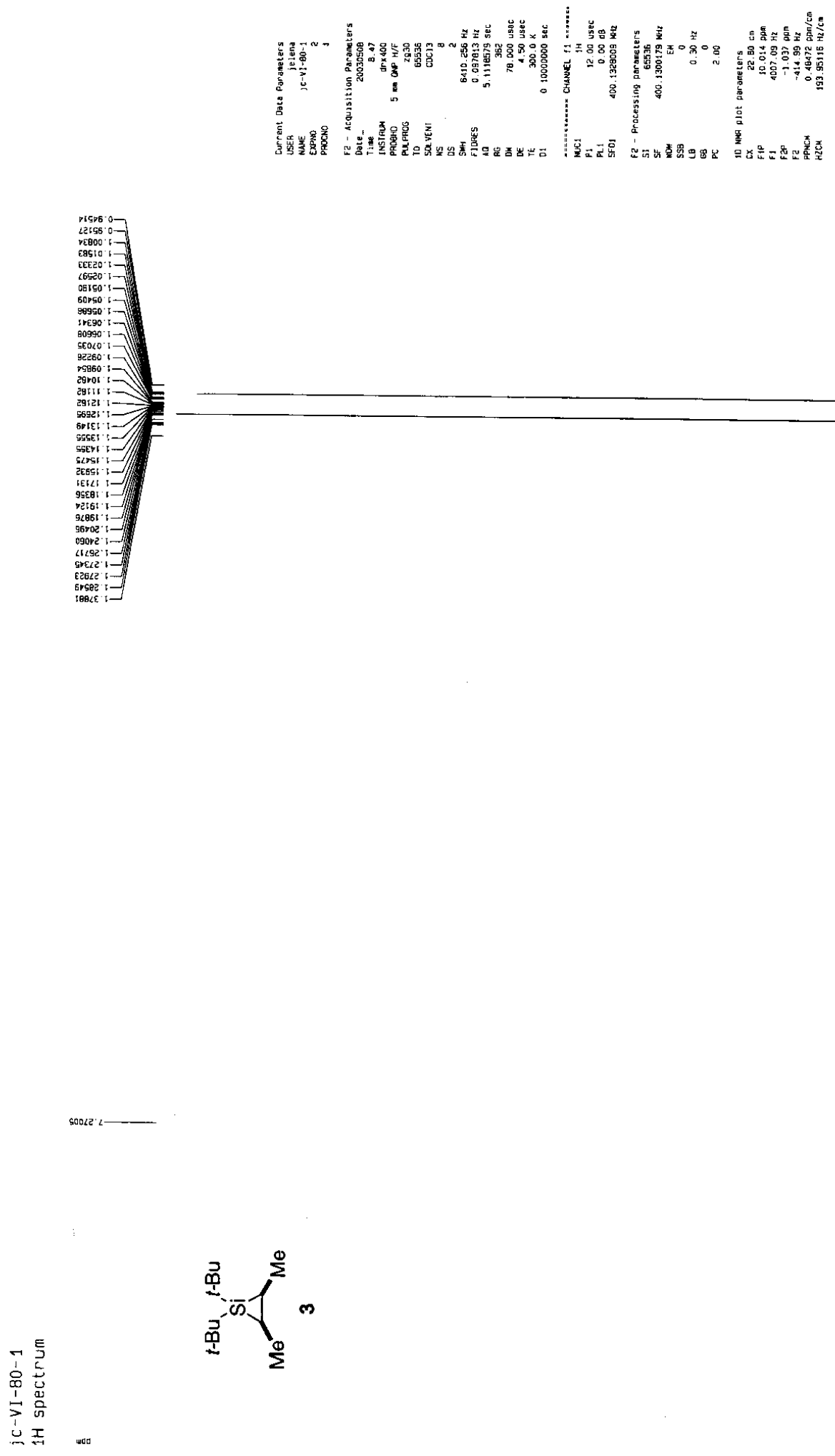


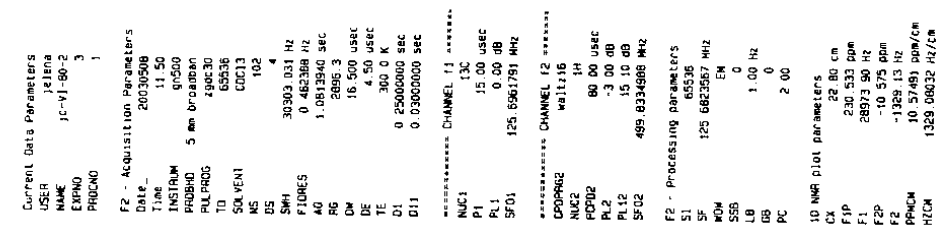

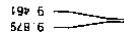

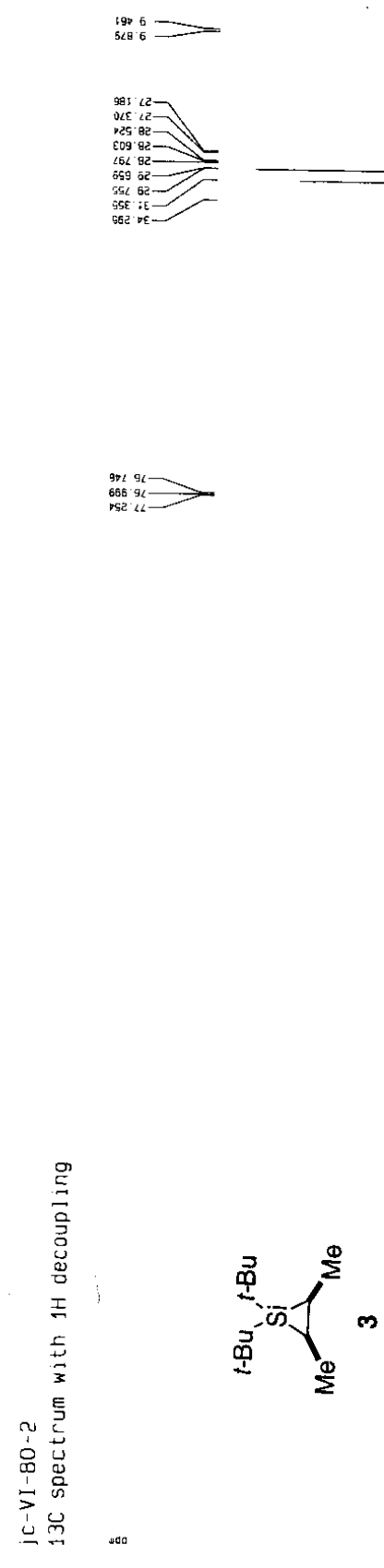

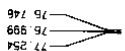

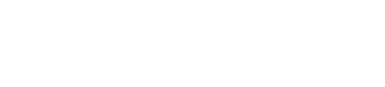

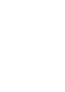




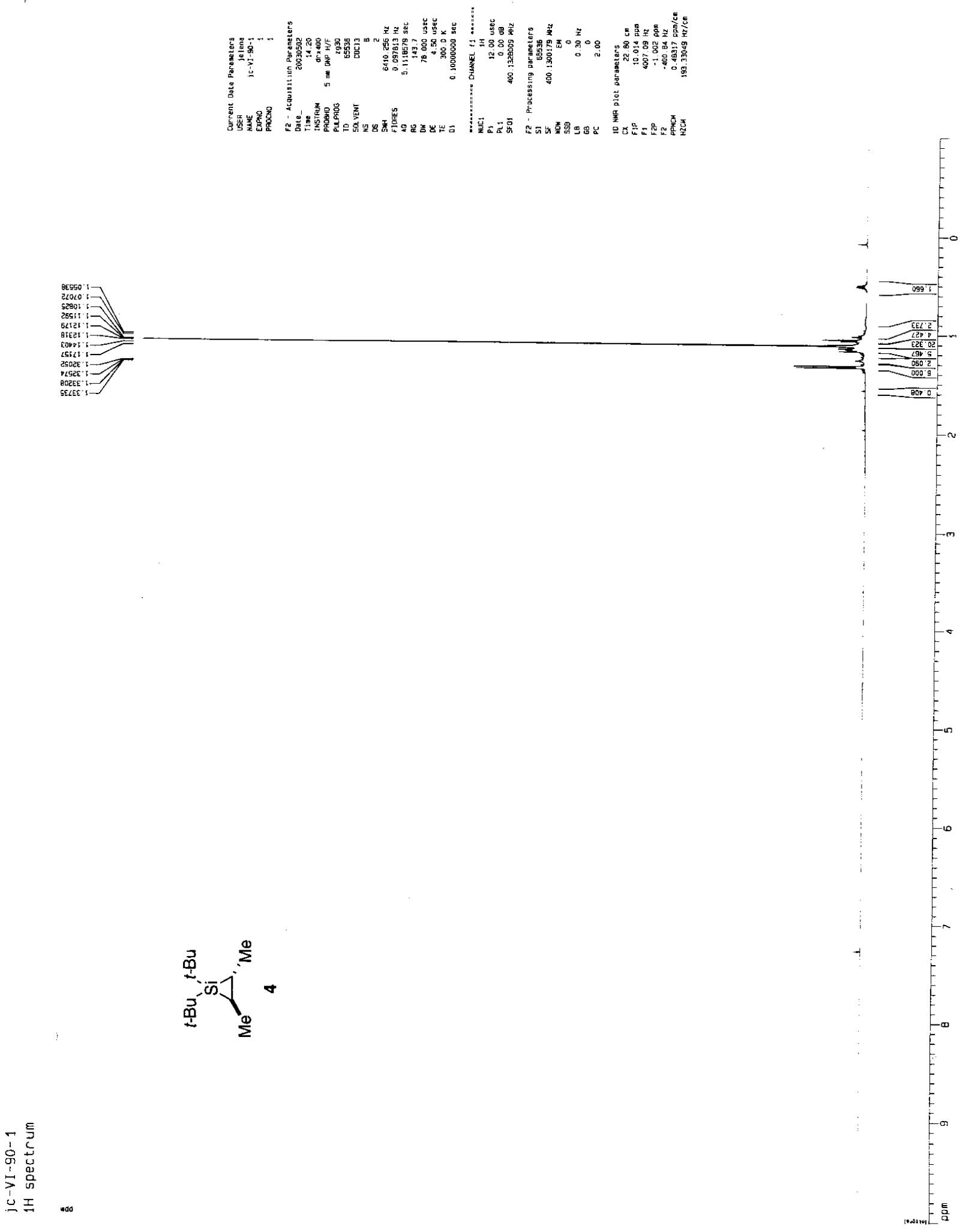




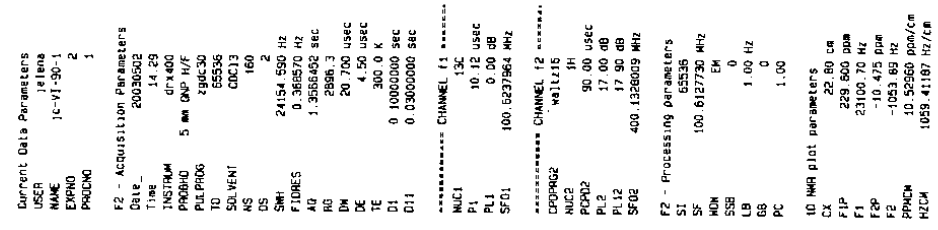

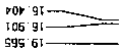

ge2 of

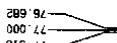

${ }_{018}^{000} 42$
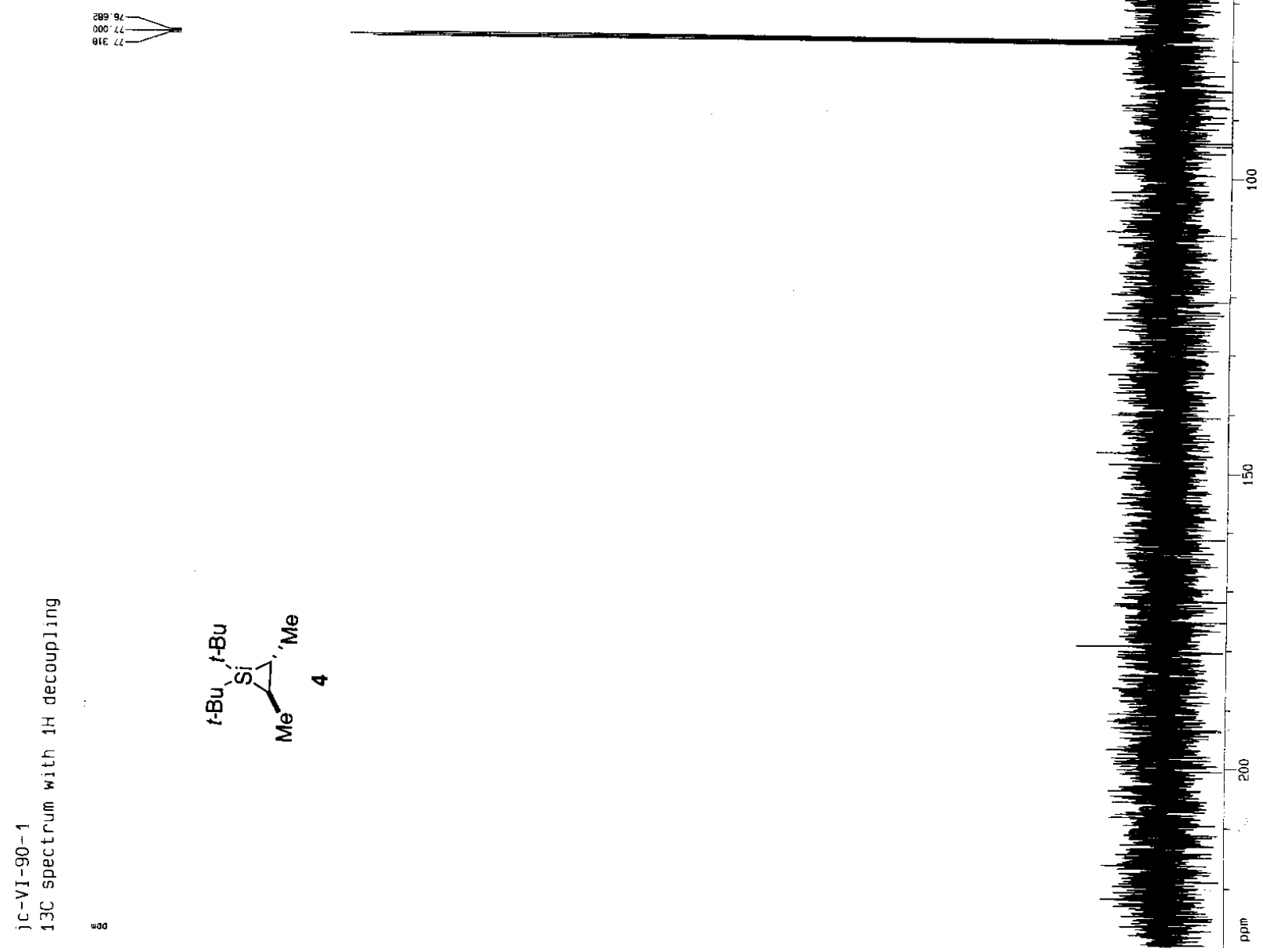


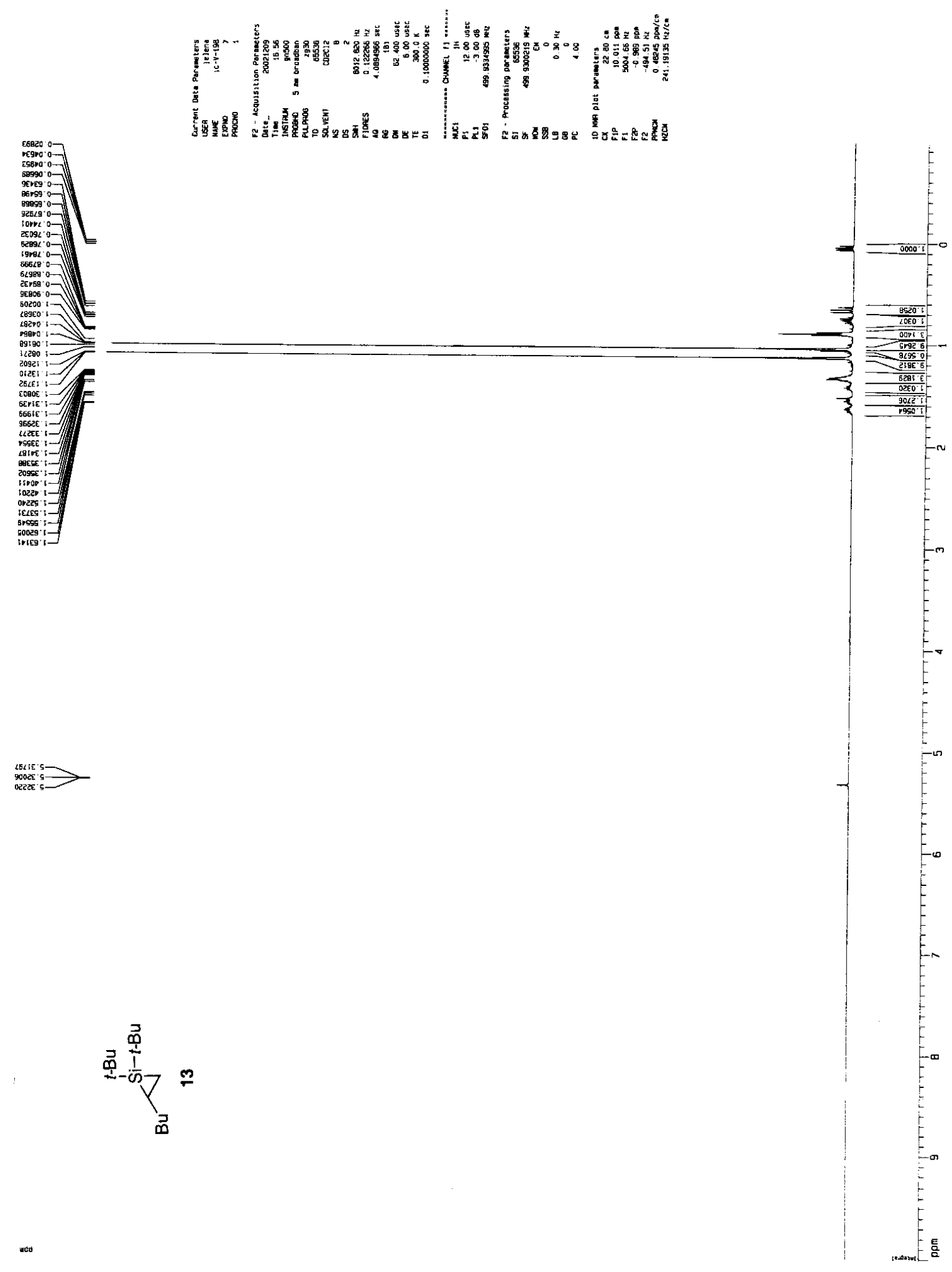



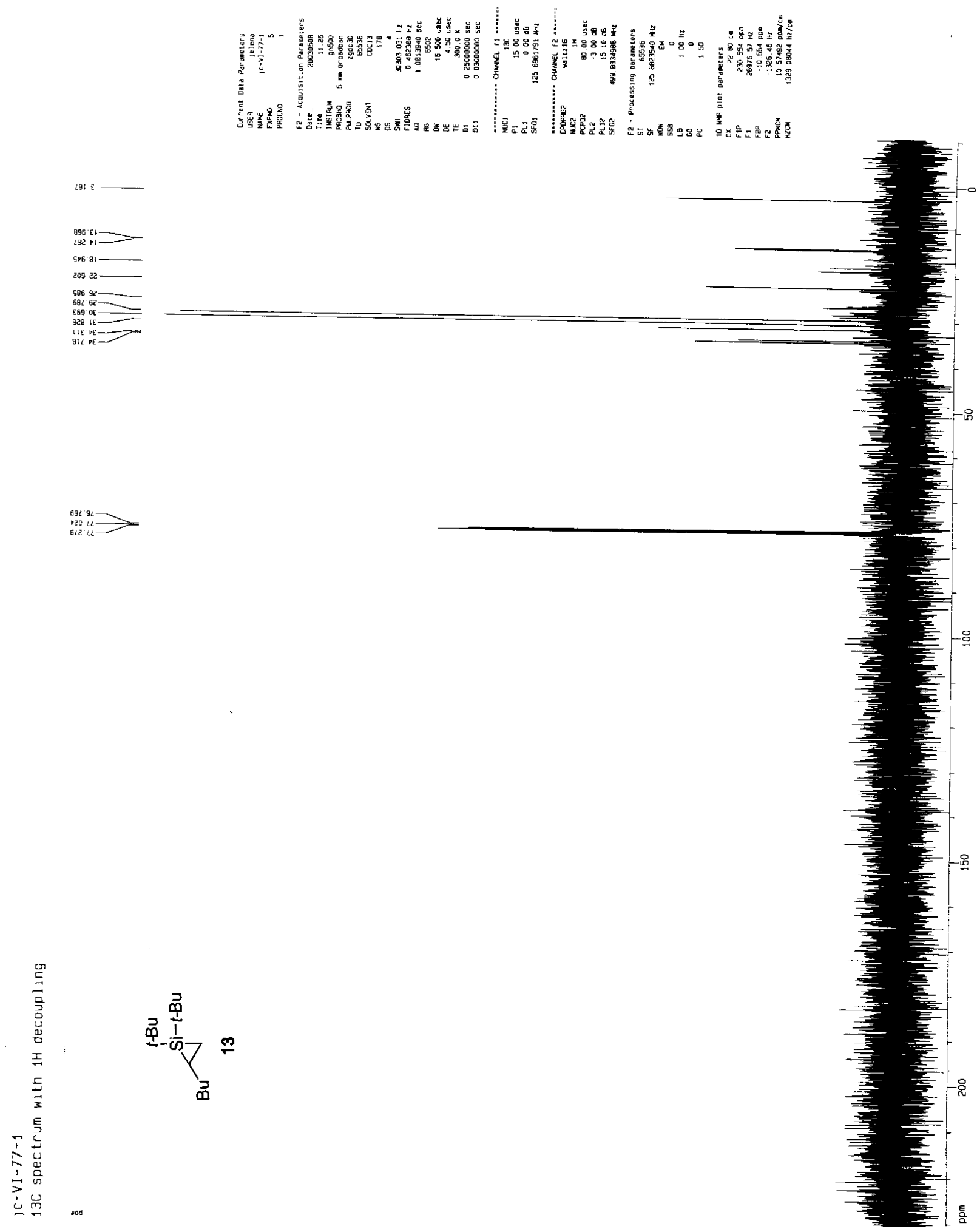


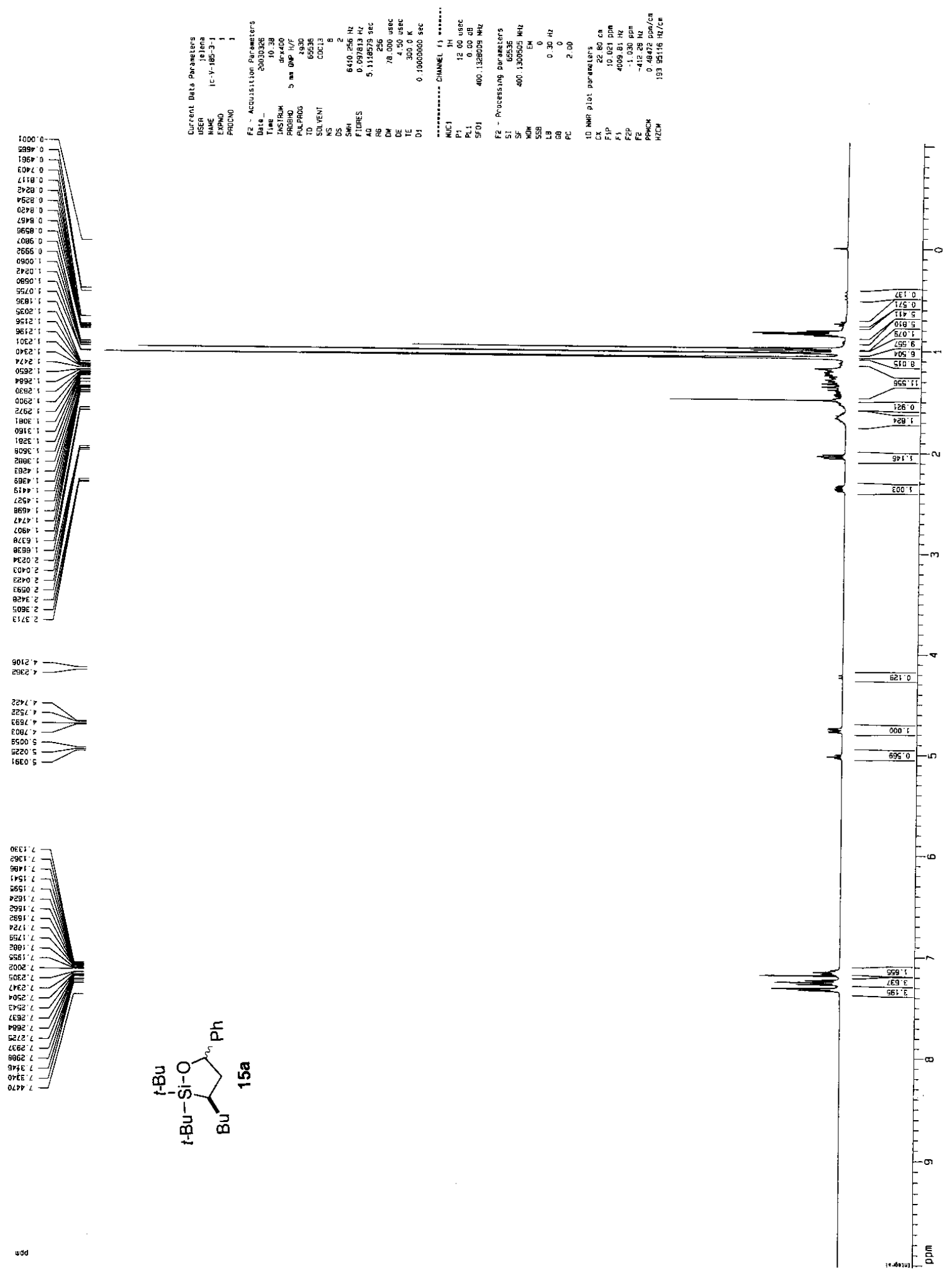



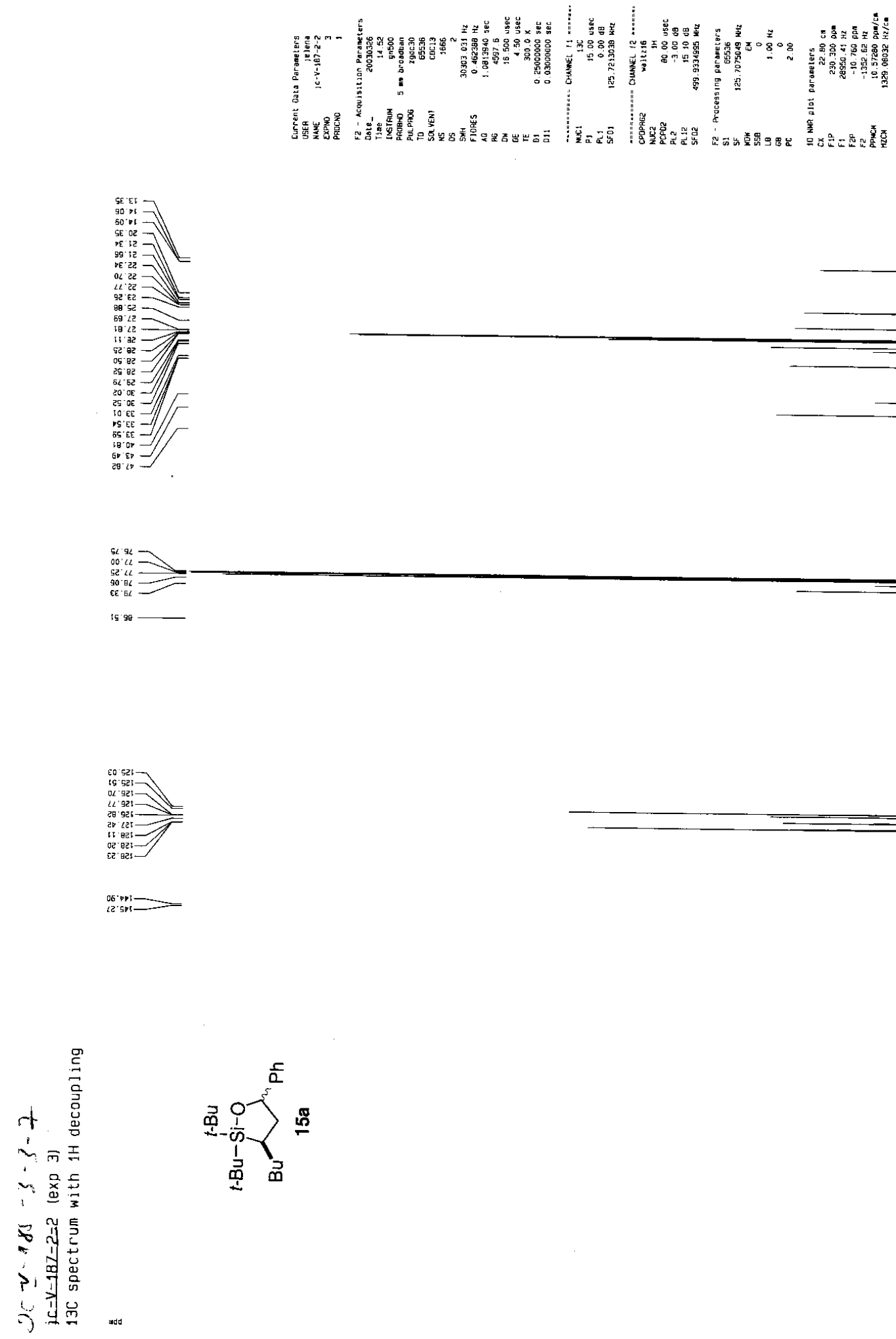

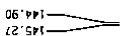
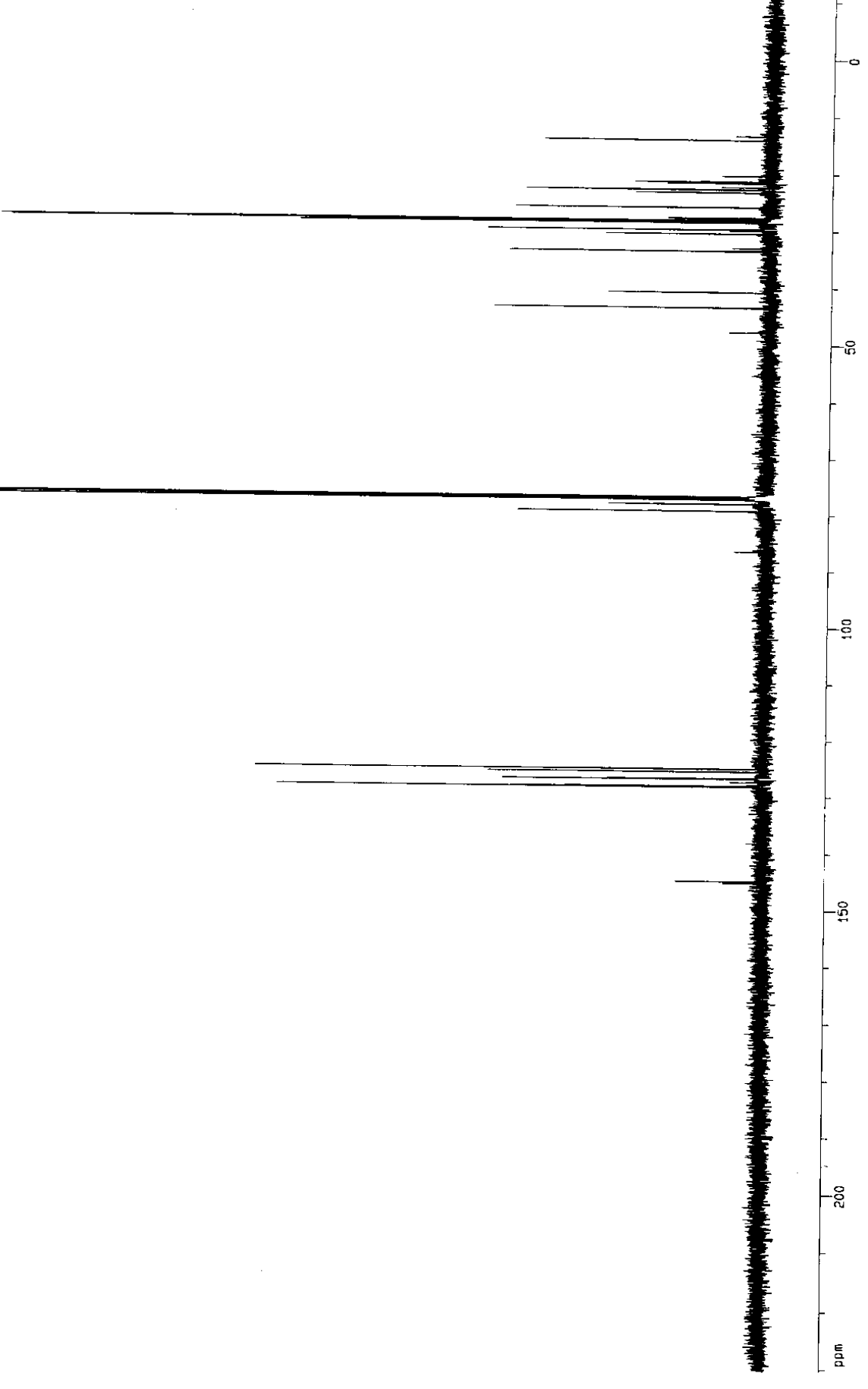


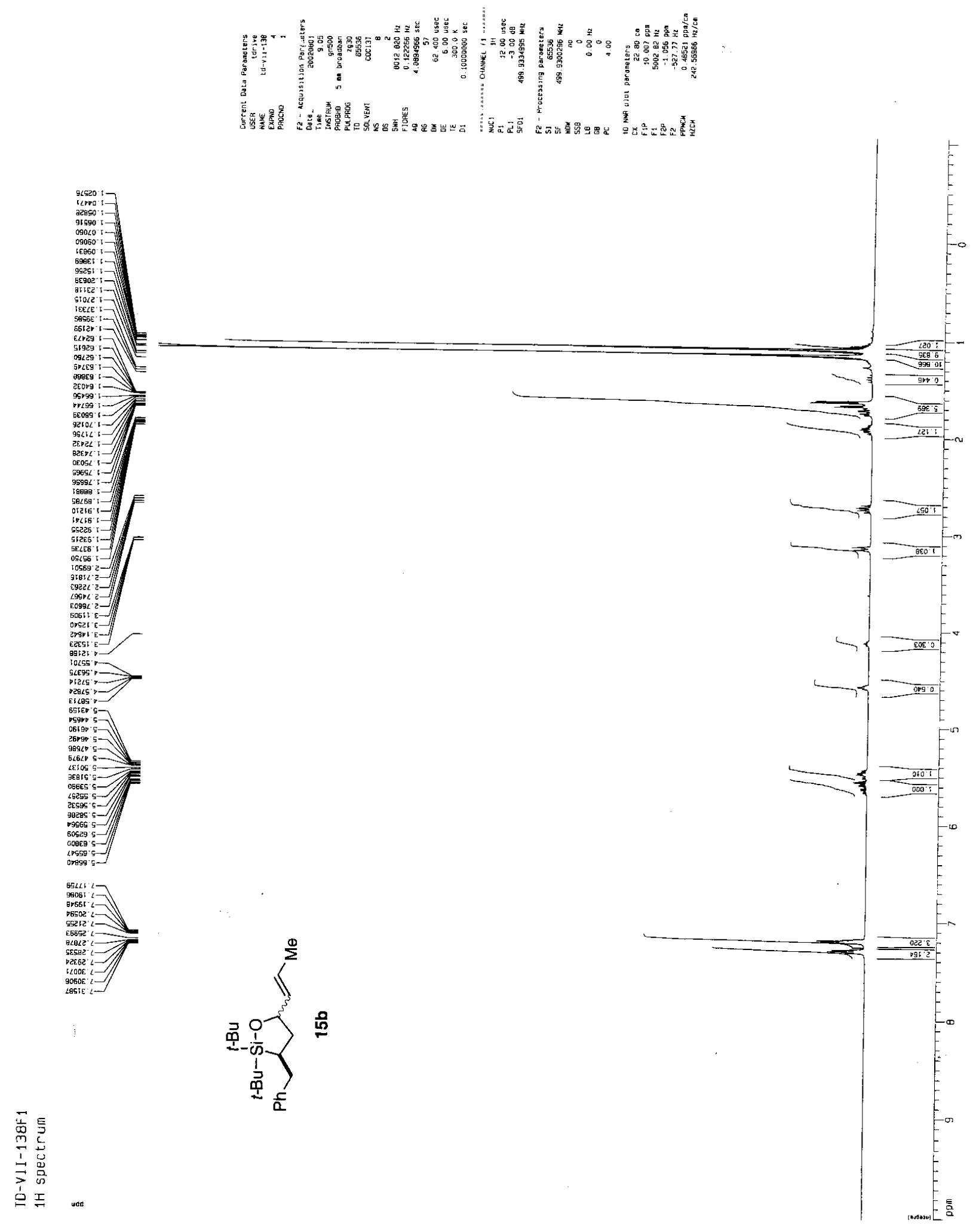




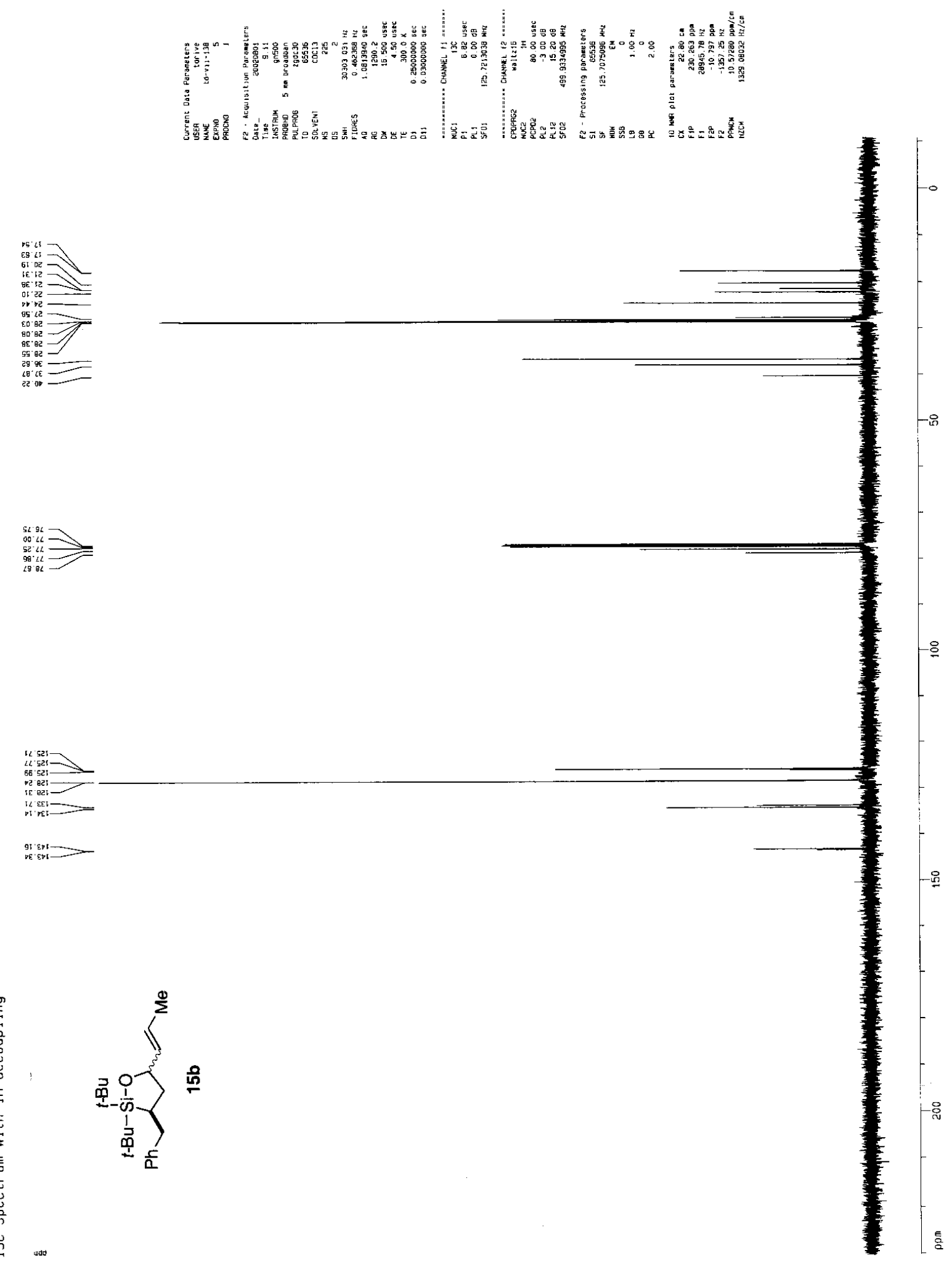




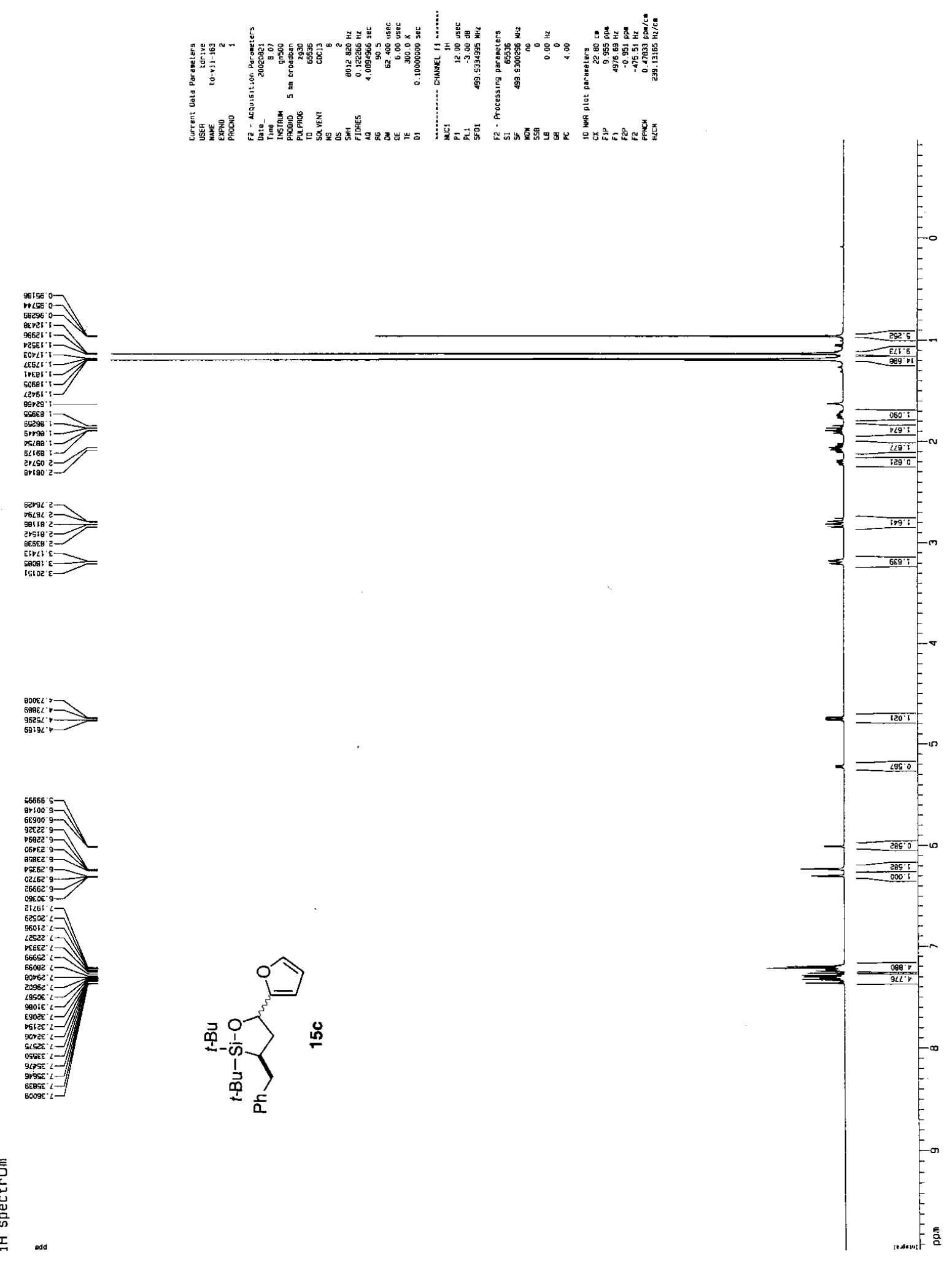




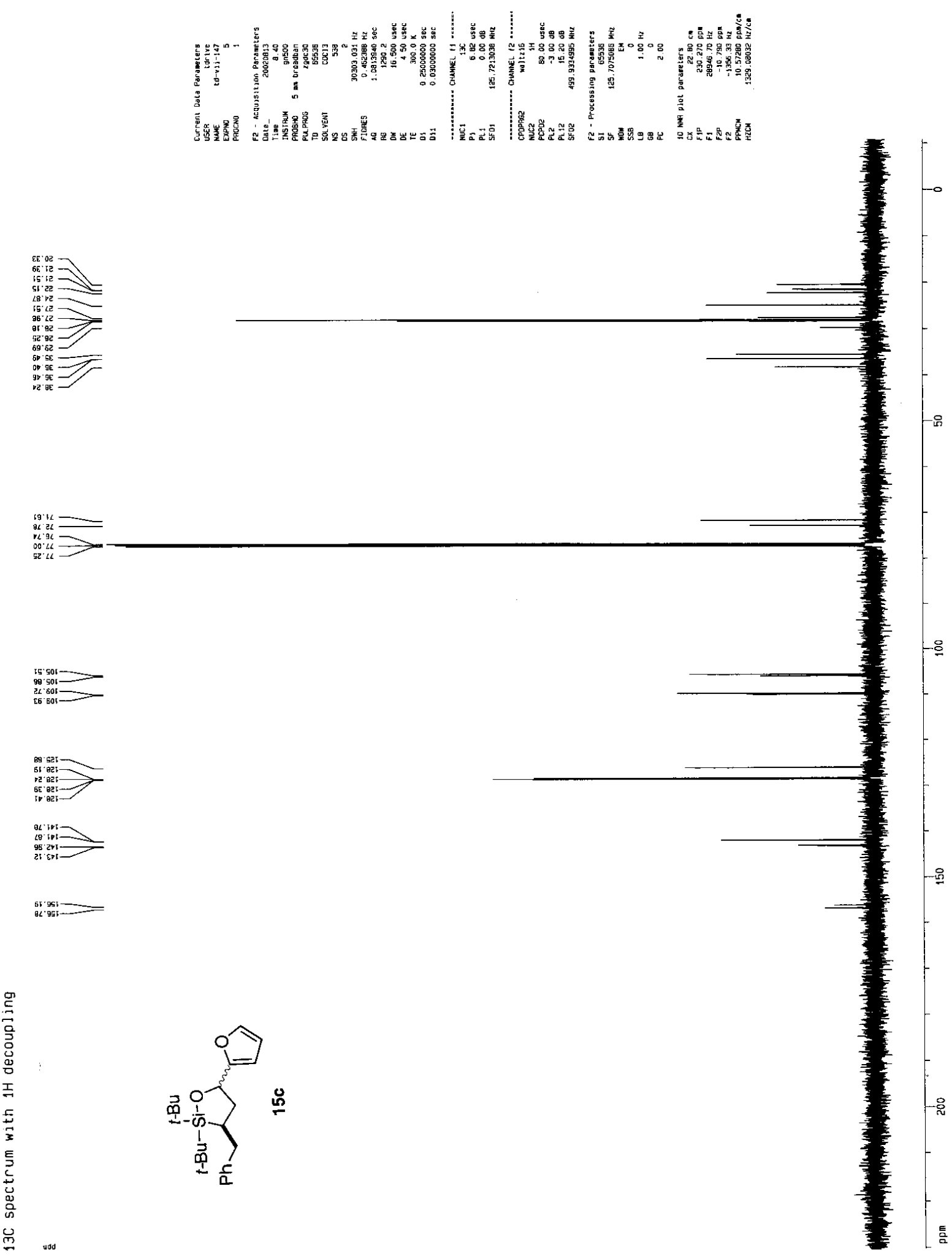



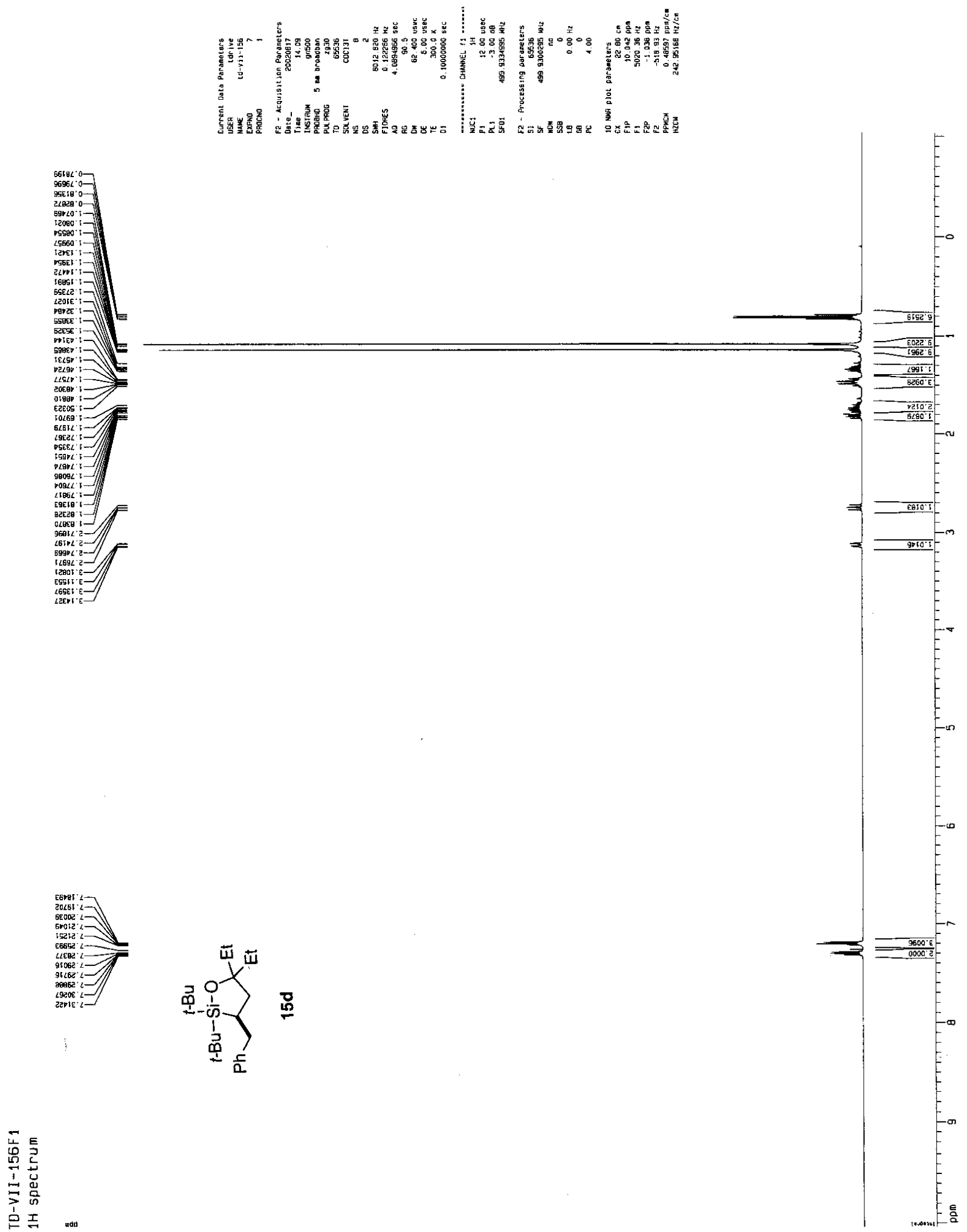


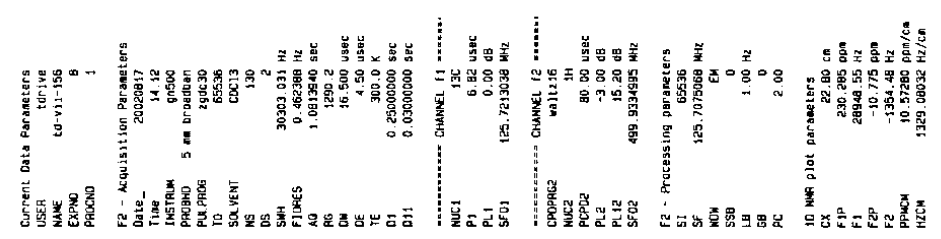

${ }_{20.6}^{92}=$

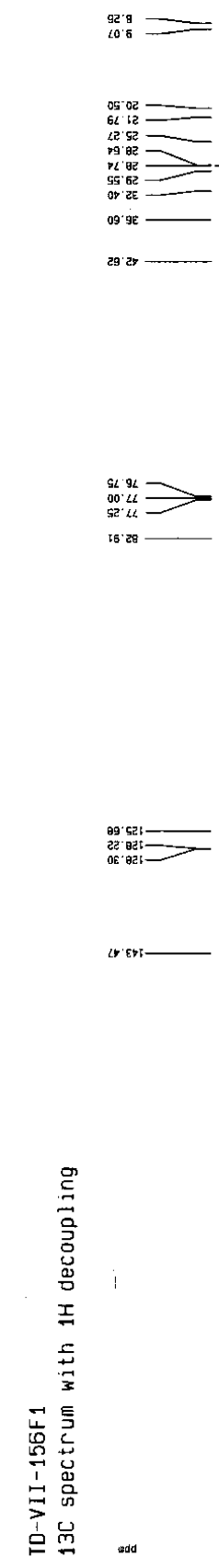

29 zs

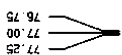

5

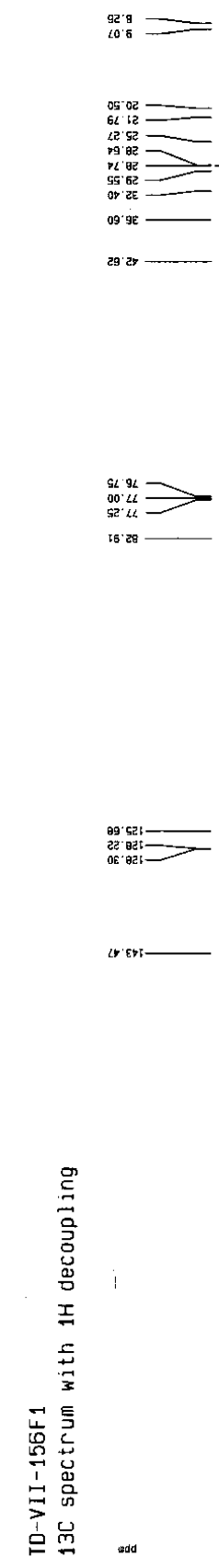

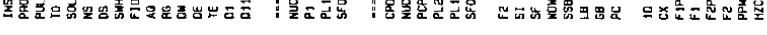
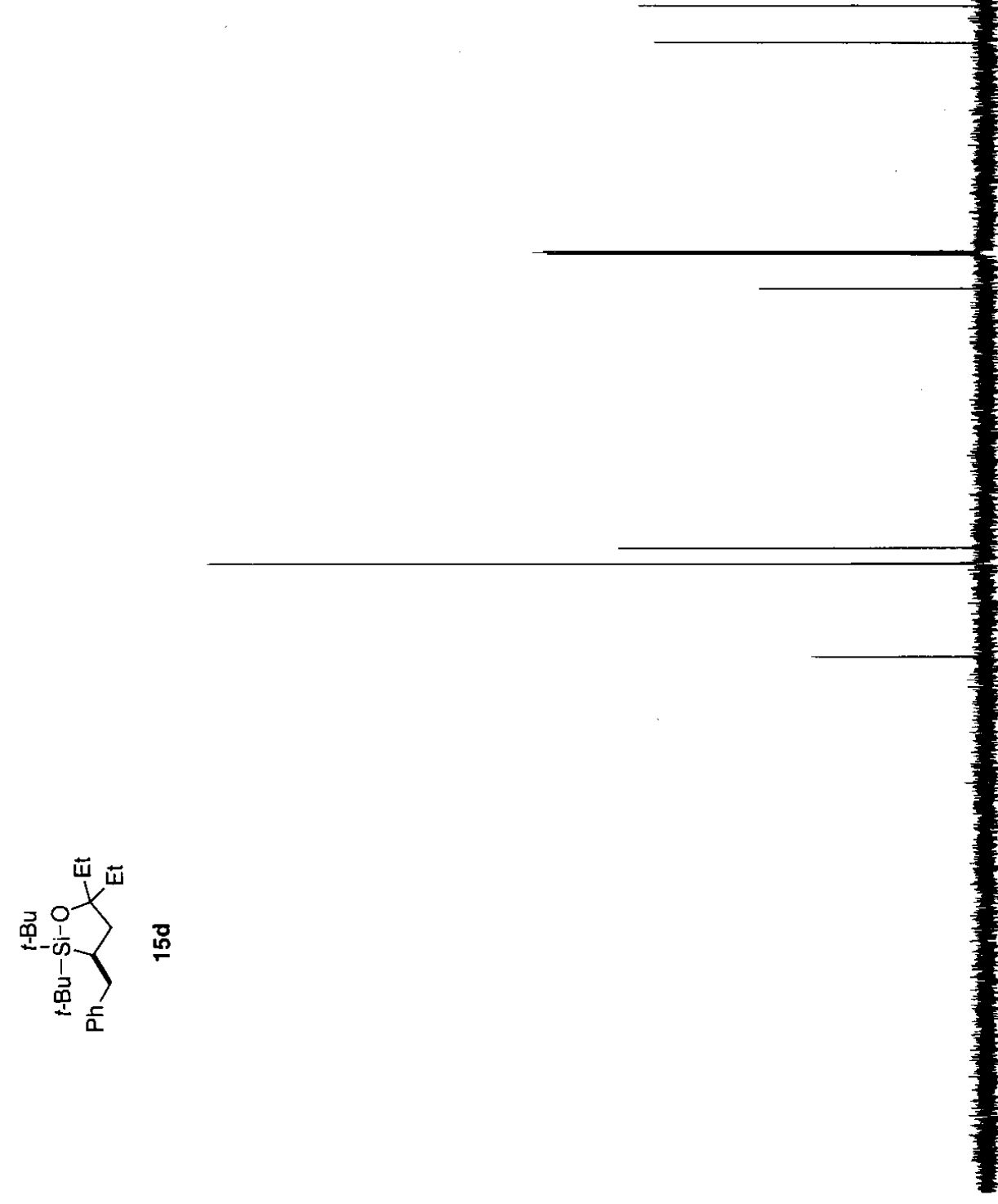

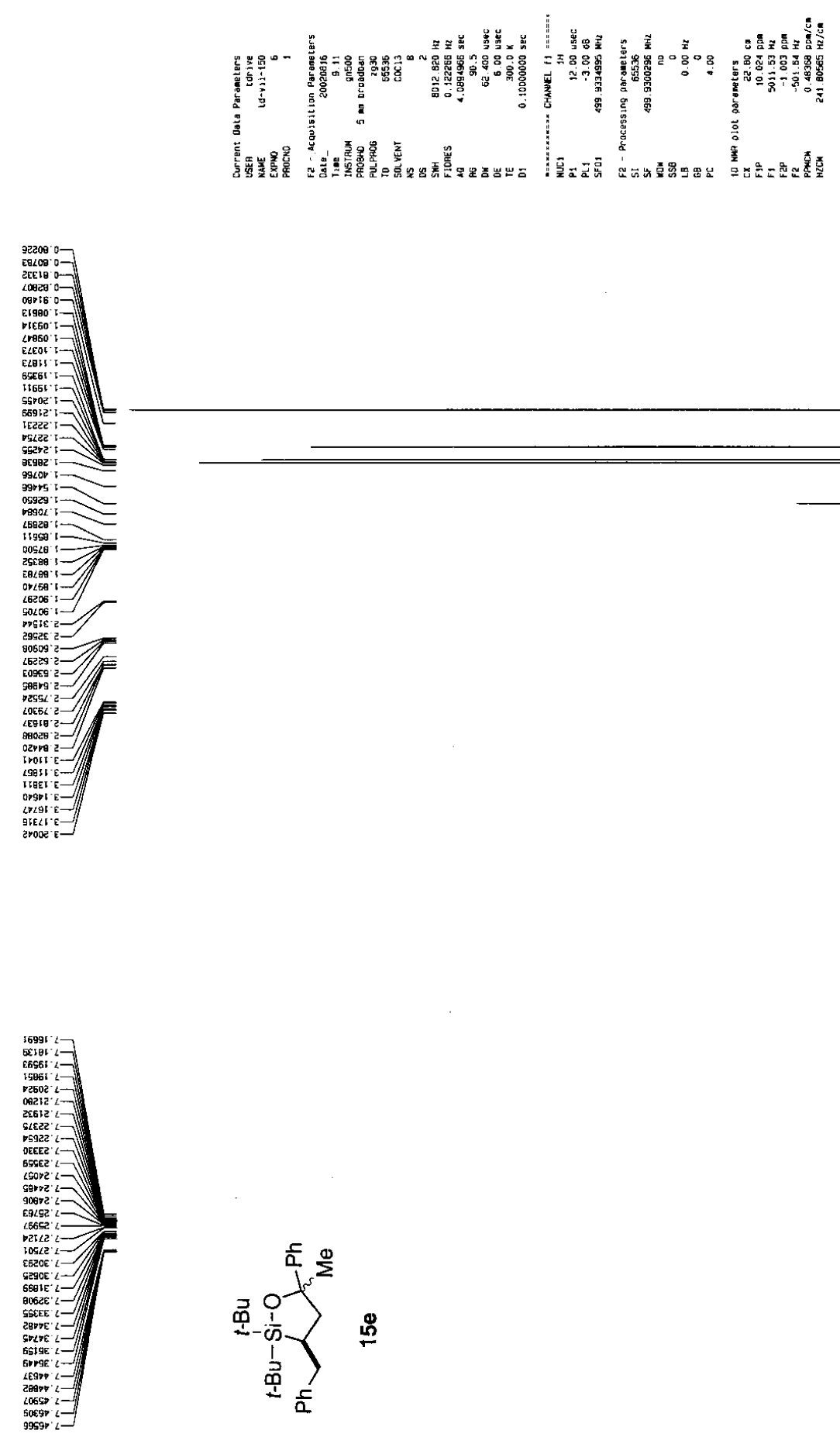

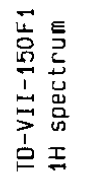




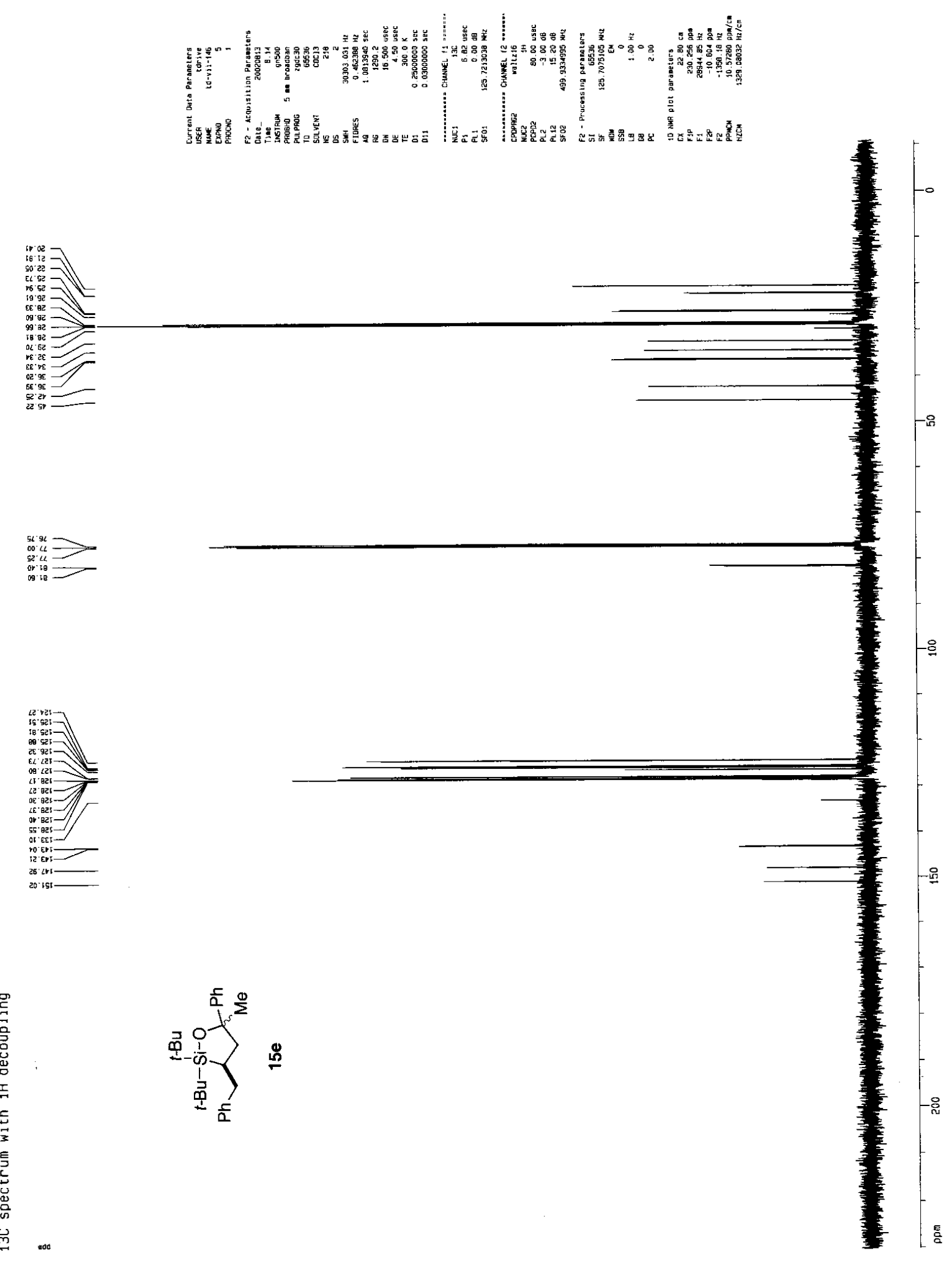



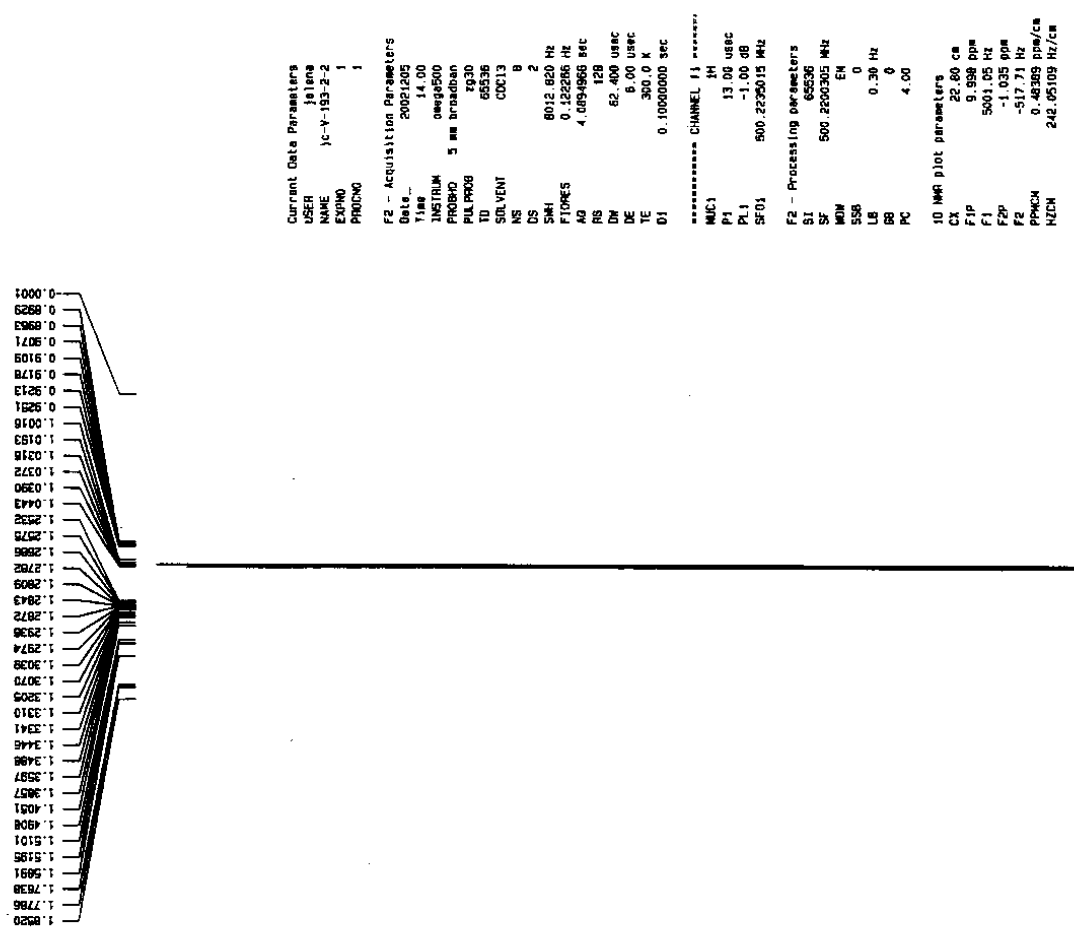

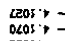

${ }_{1025}^{2025}:$
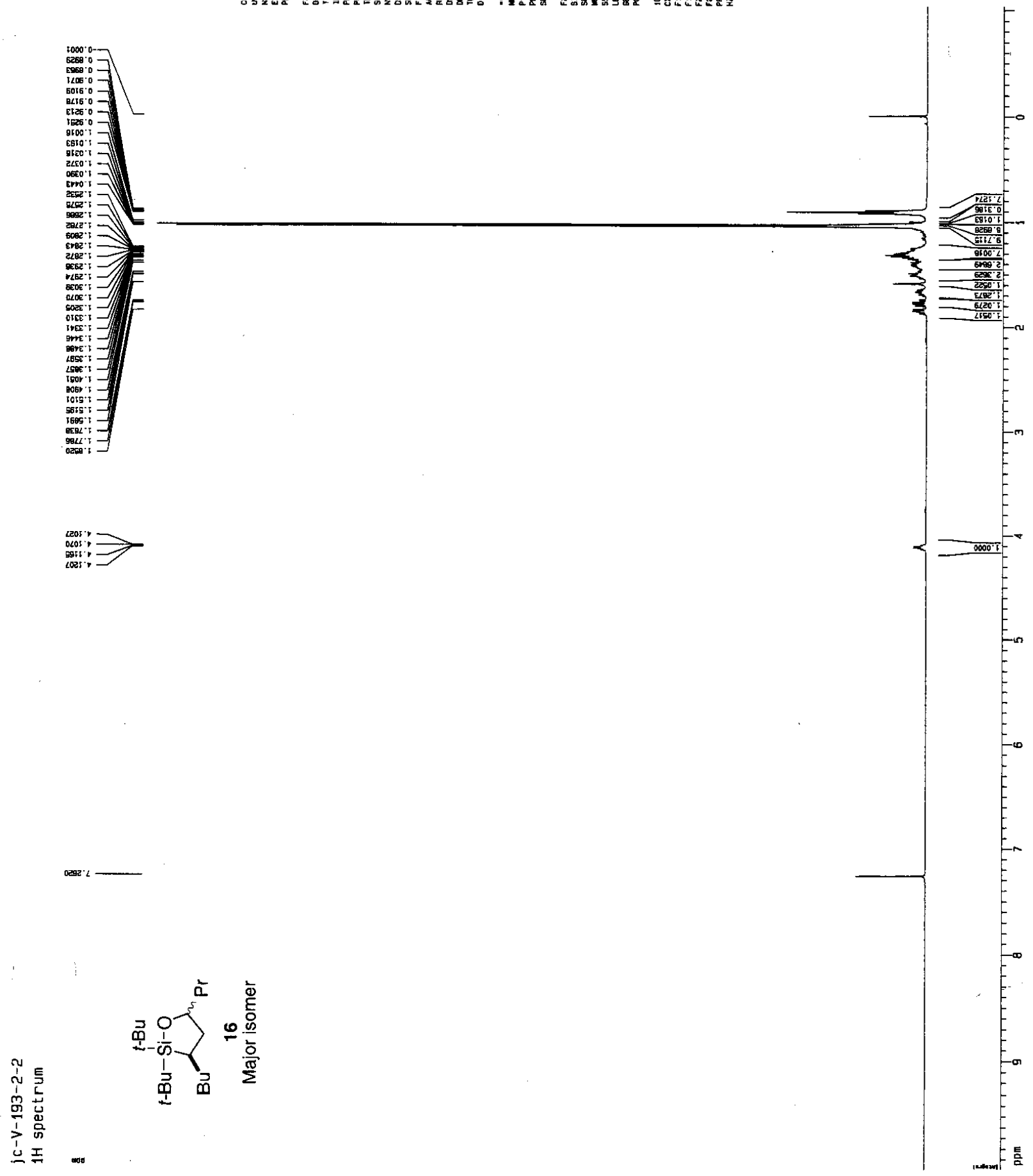

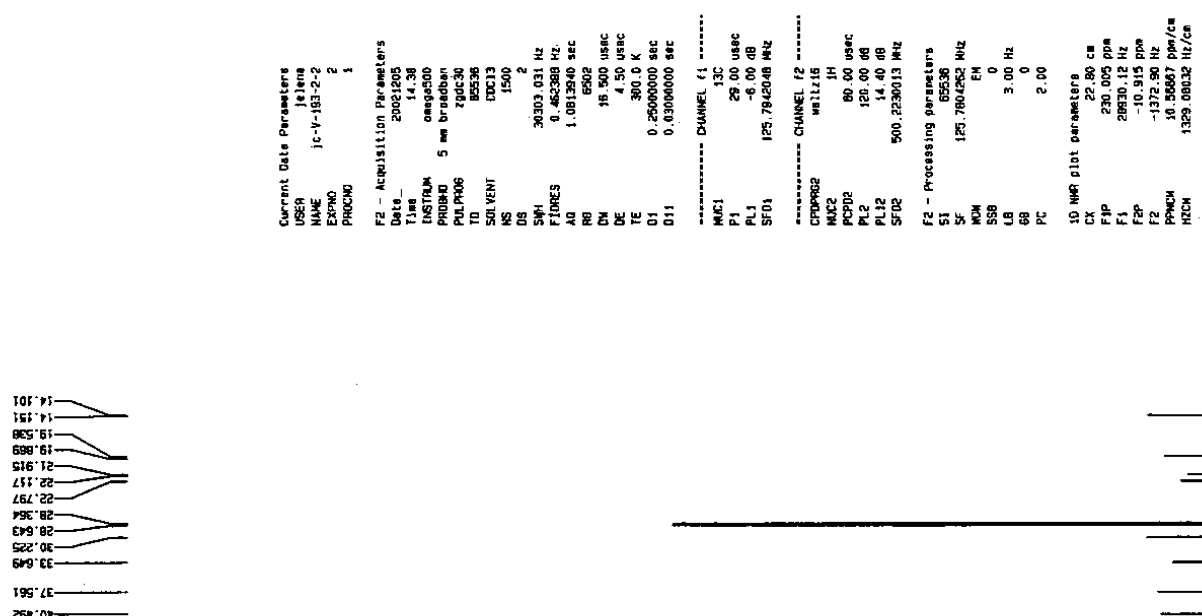

ใ⿺廴⿻肀二𠃋
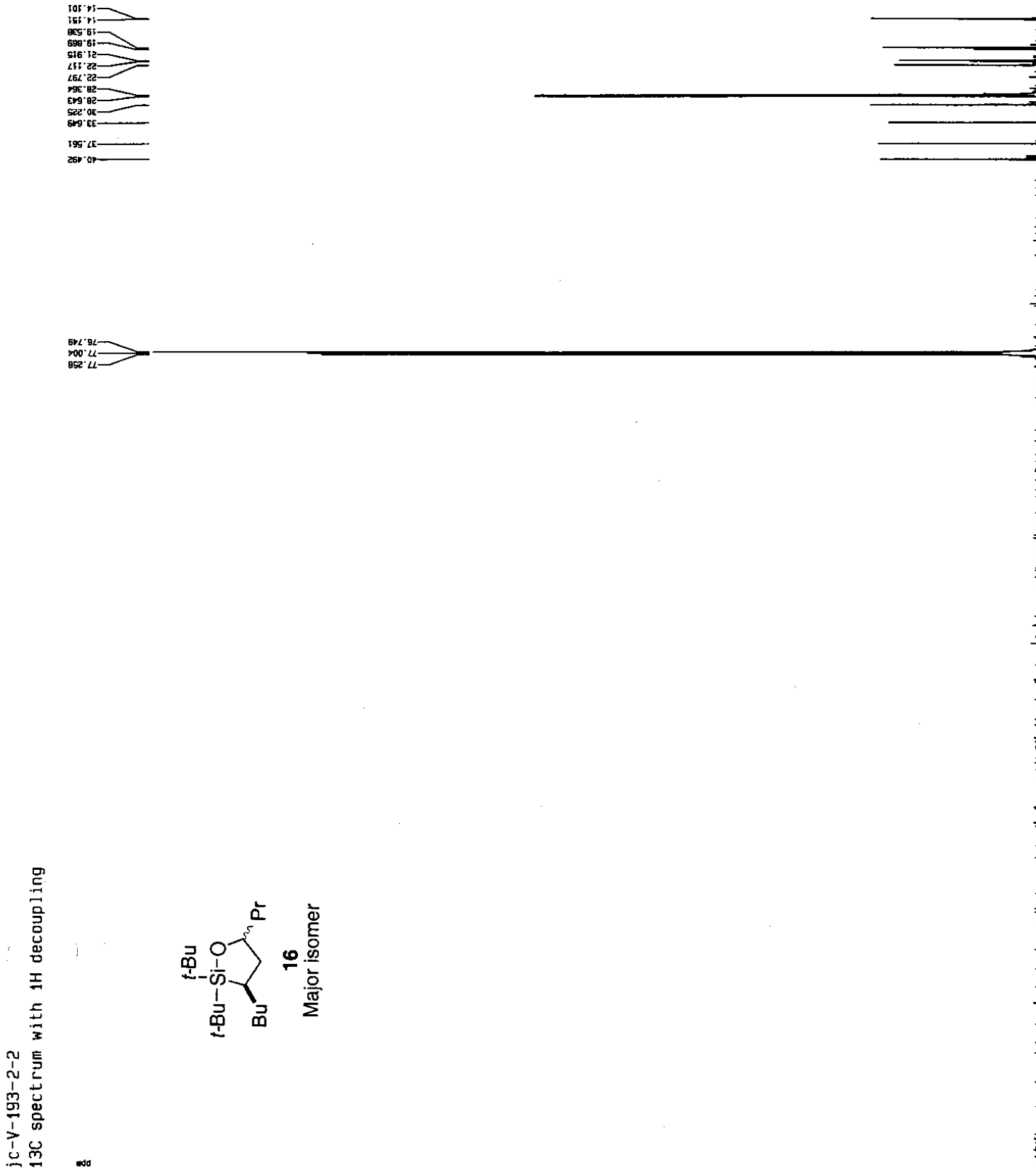

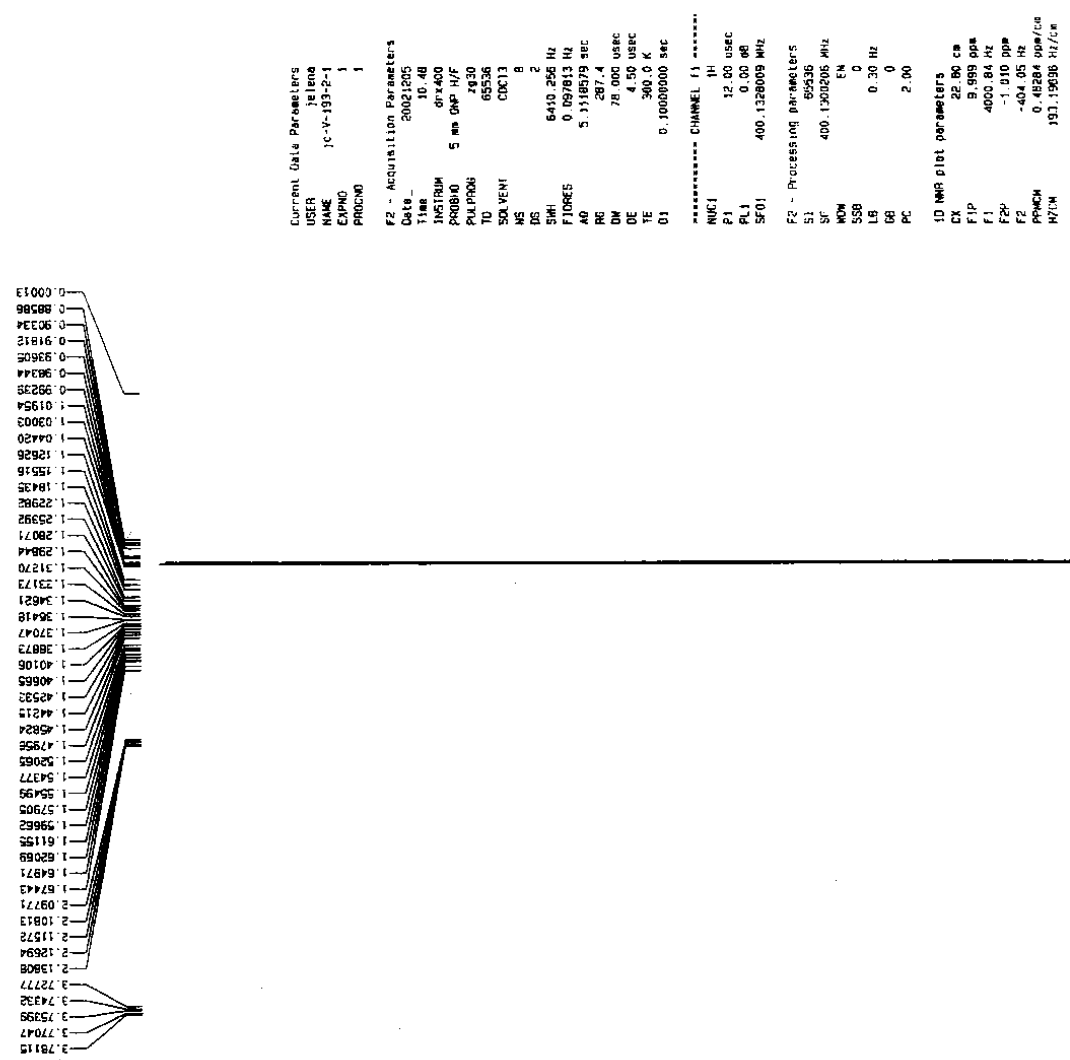

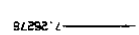
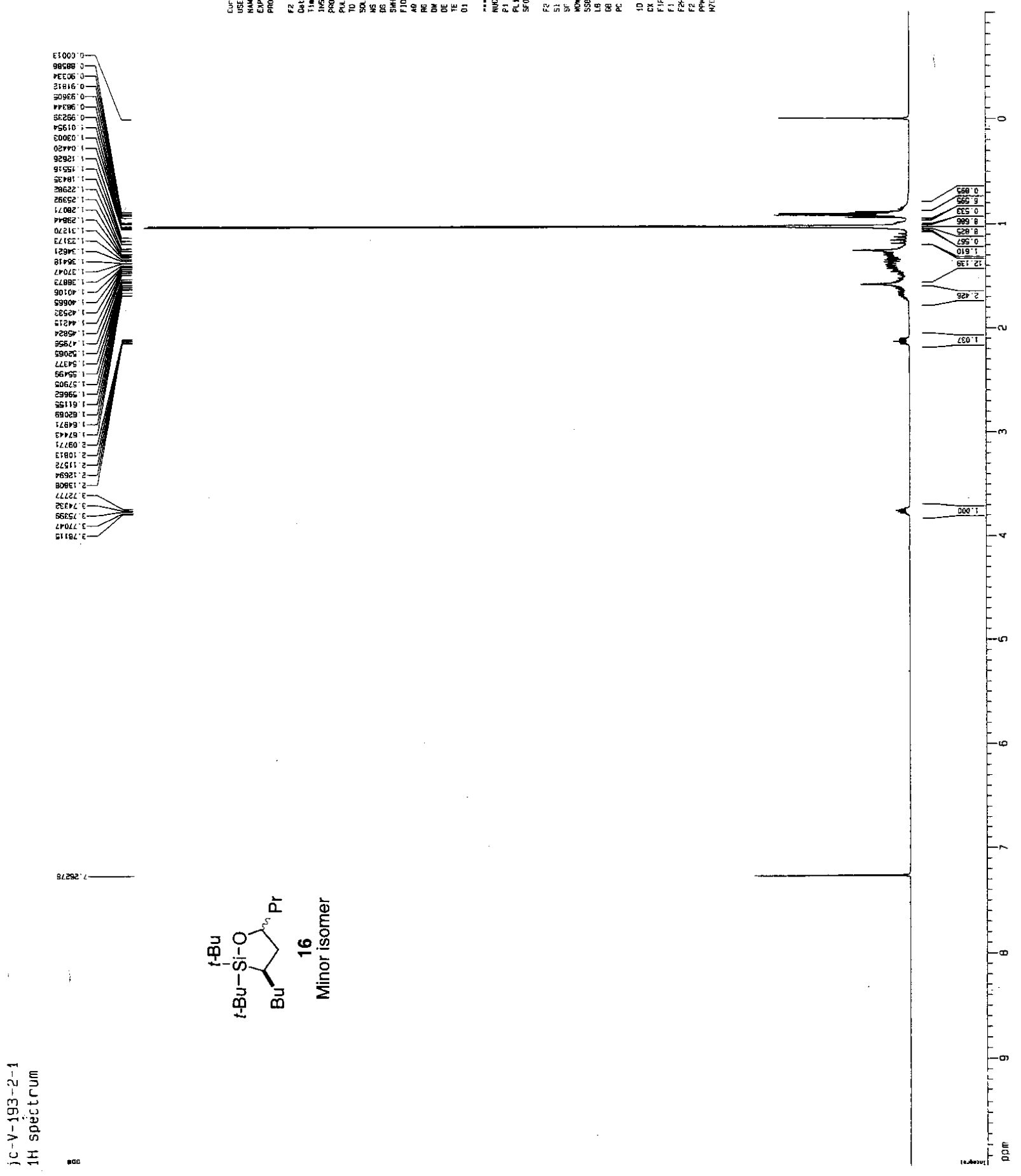

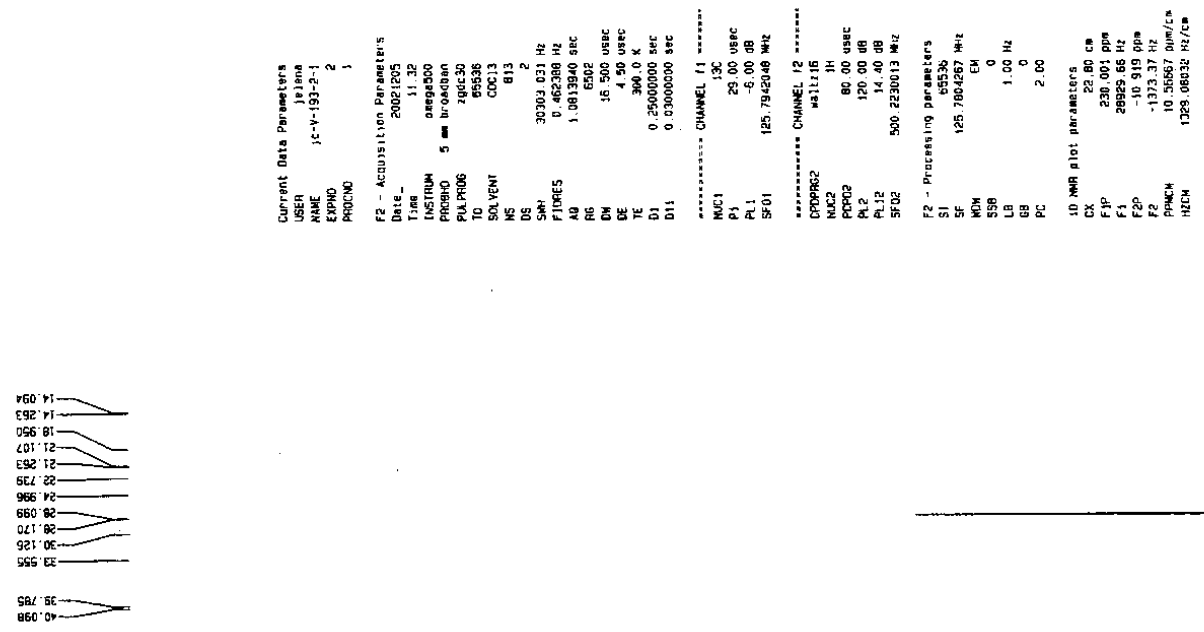

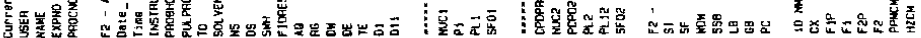
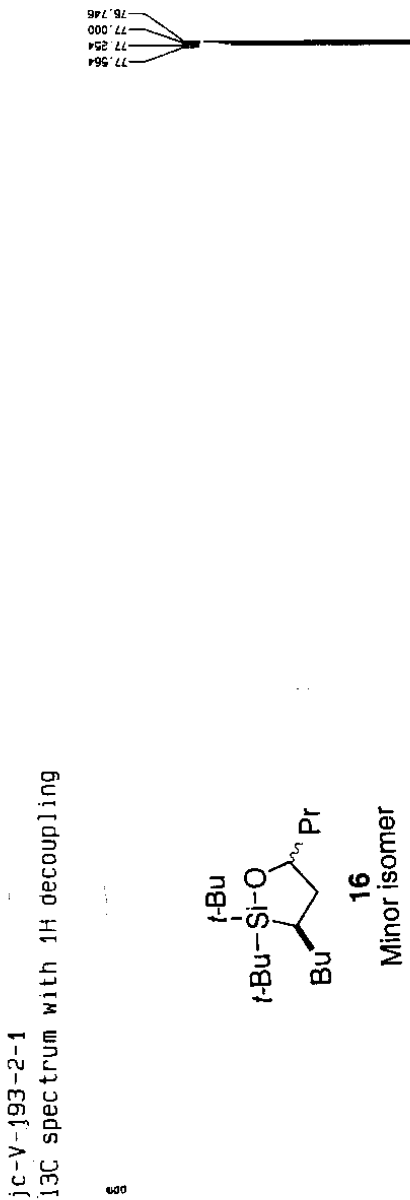


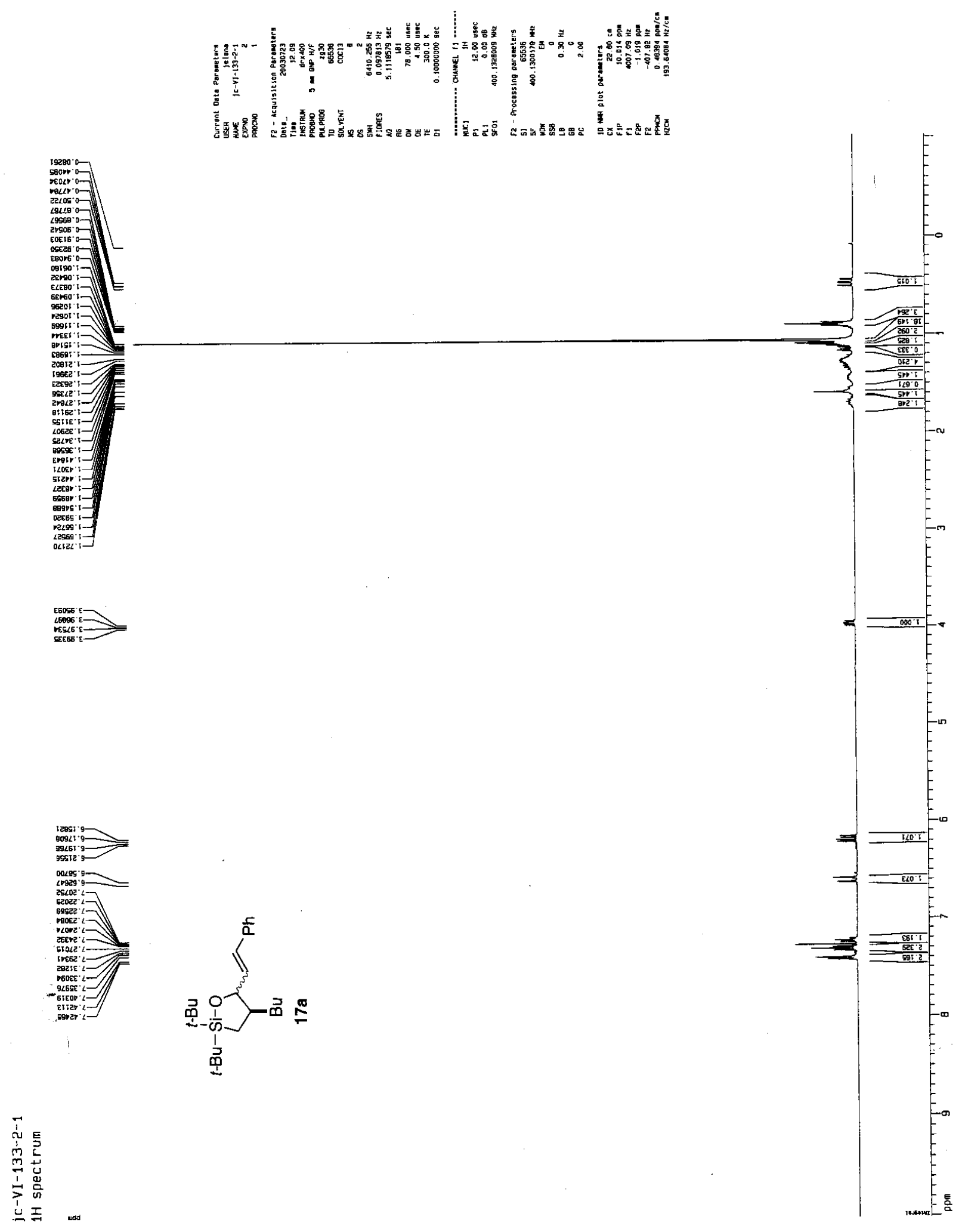




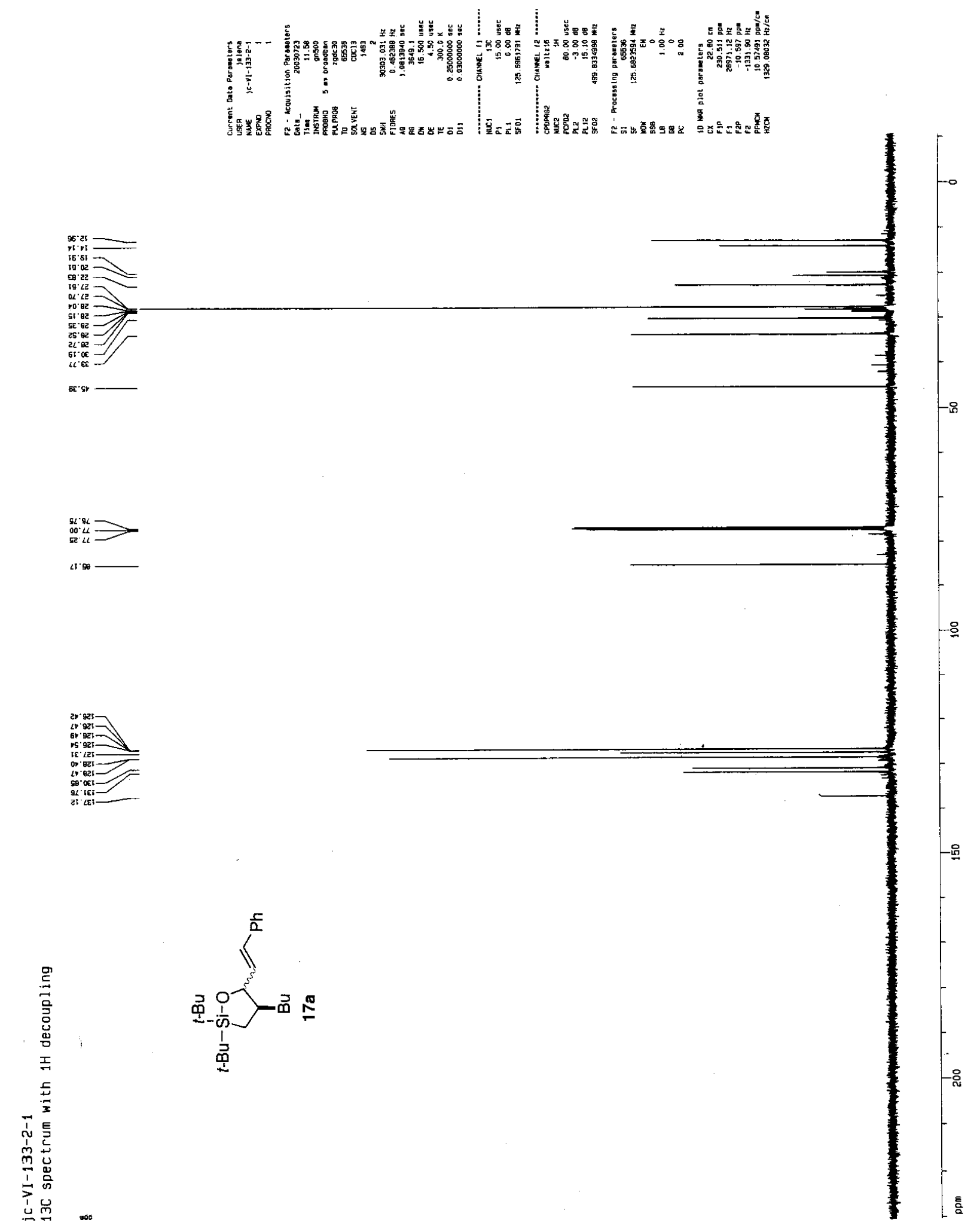




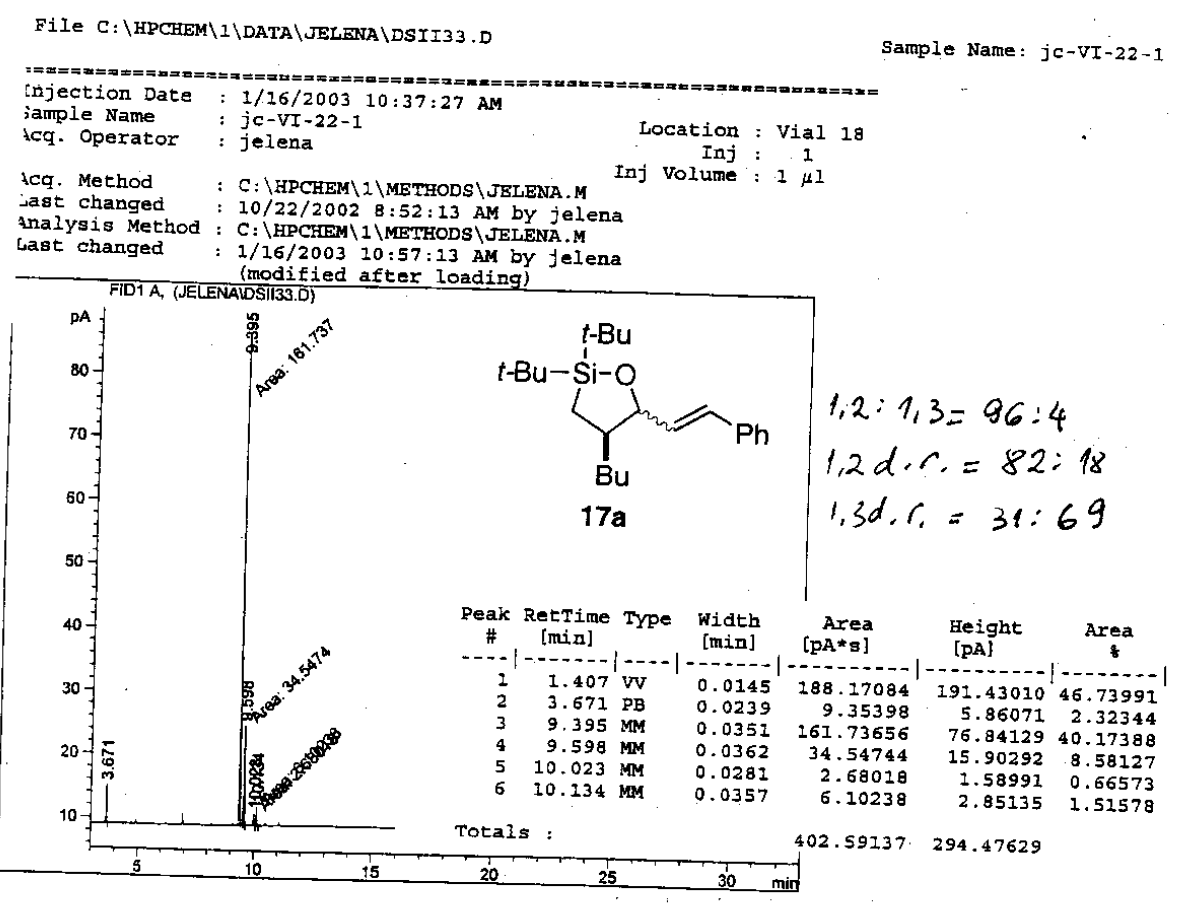



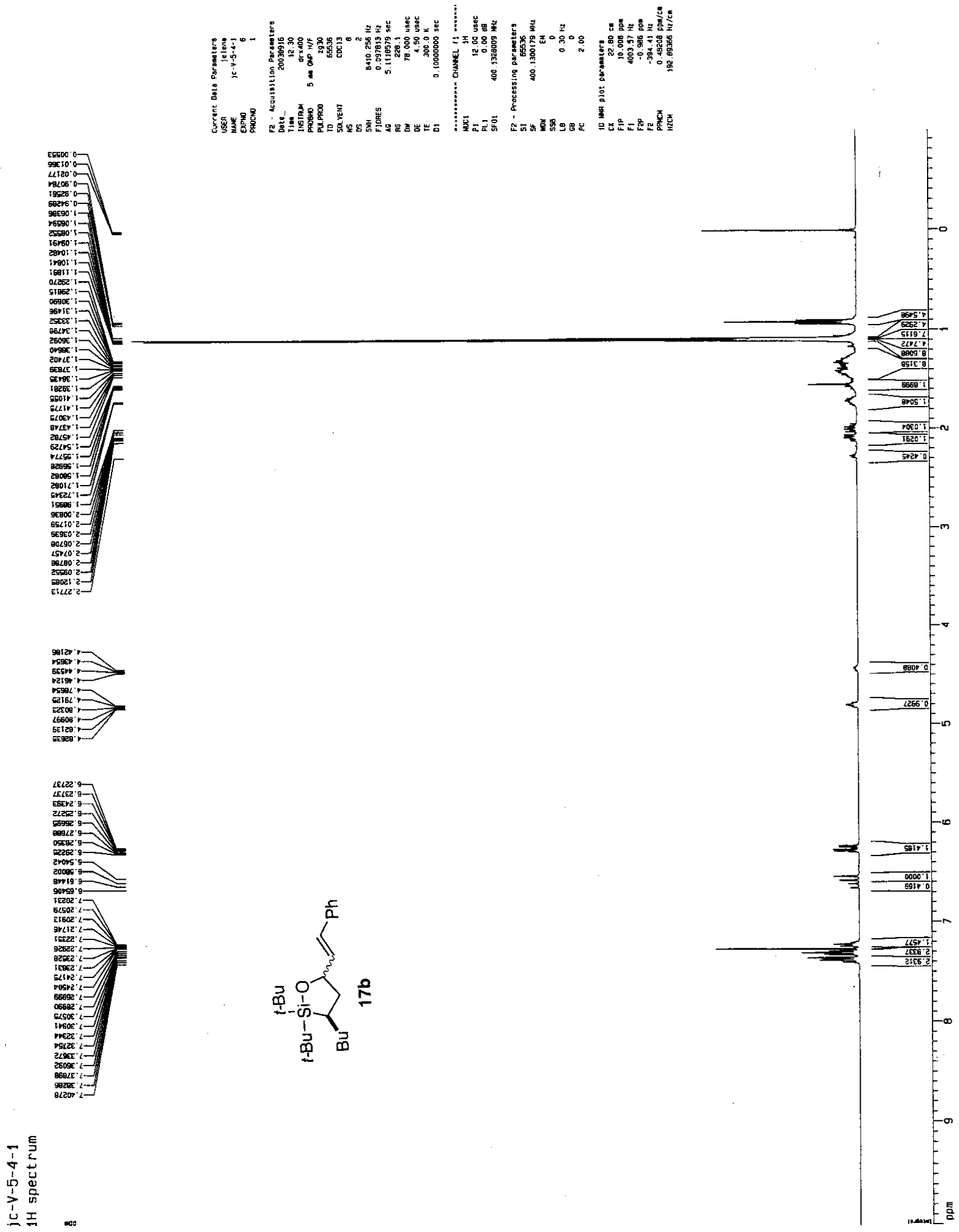


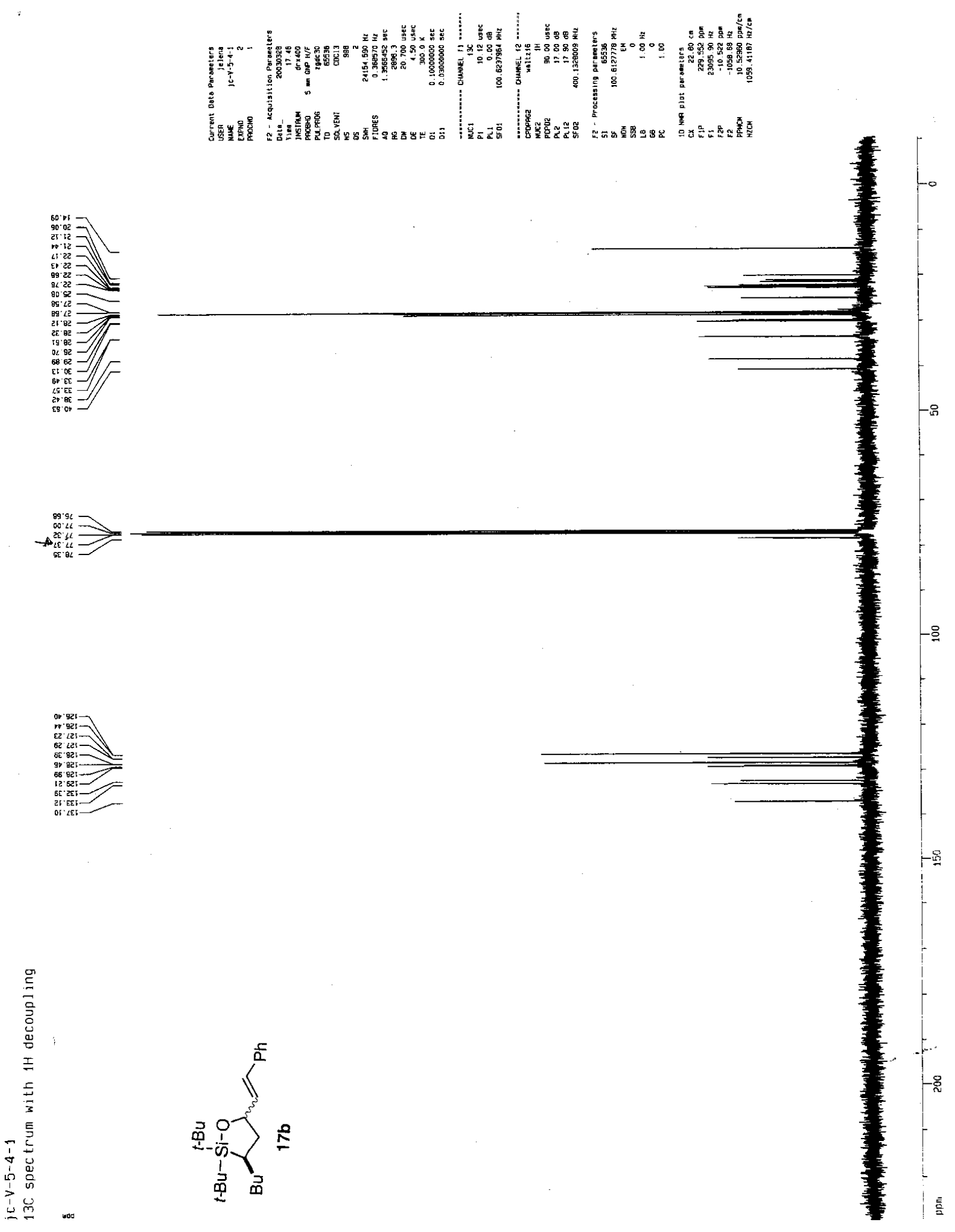


Data File C: \HPCHEM \I\DATA \JELENA \JCVI147.D
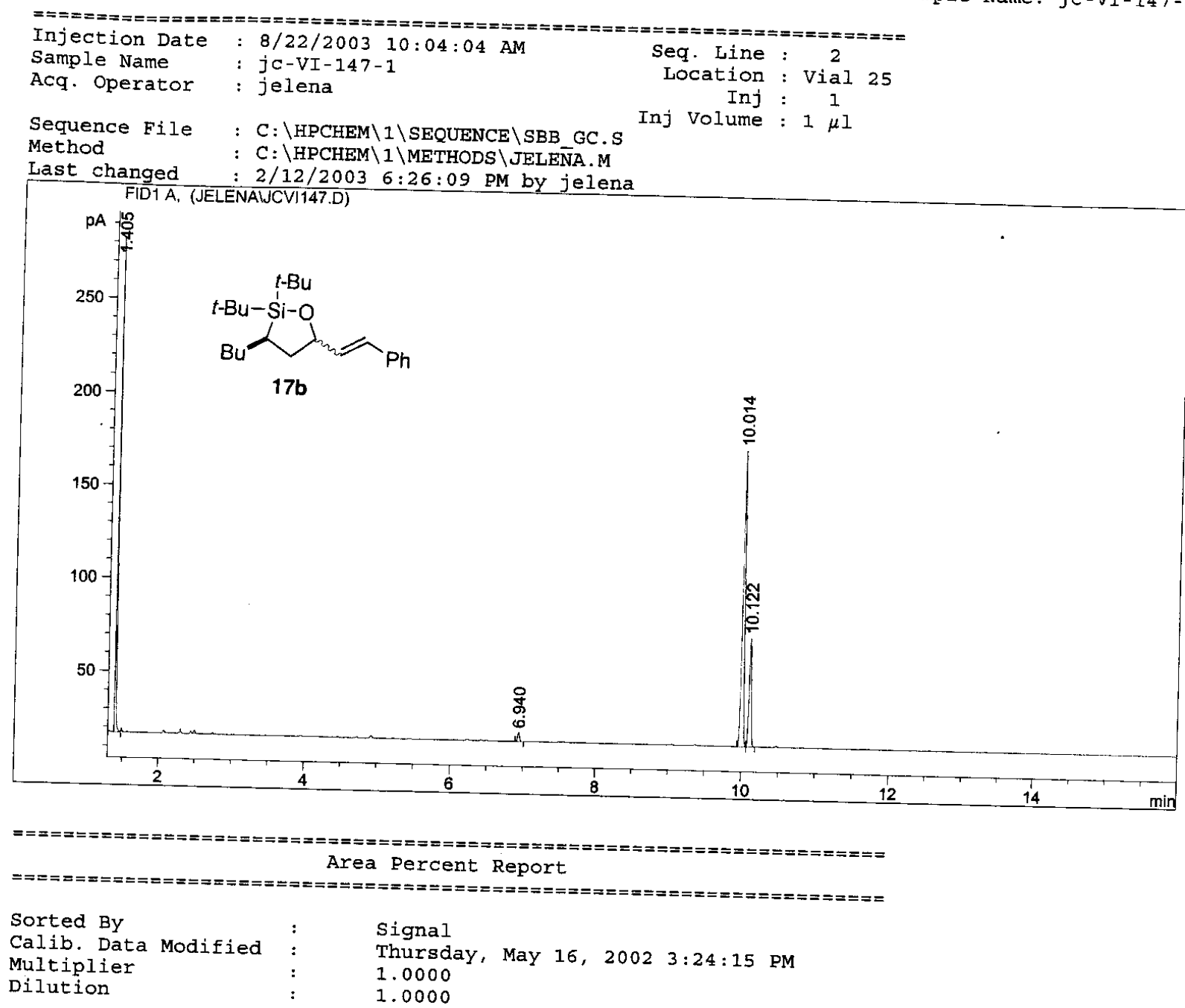

Signal 1: FIDI A,

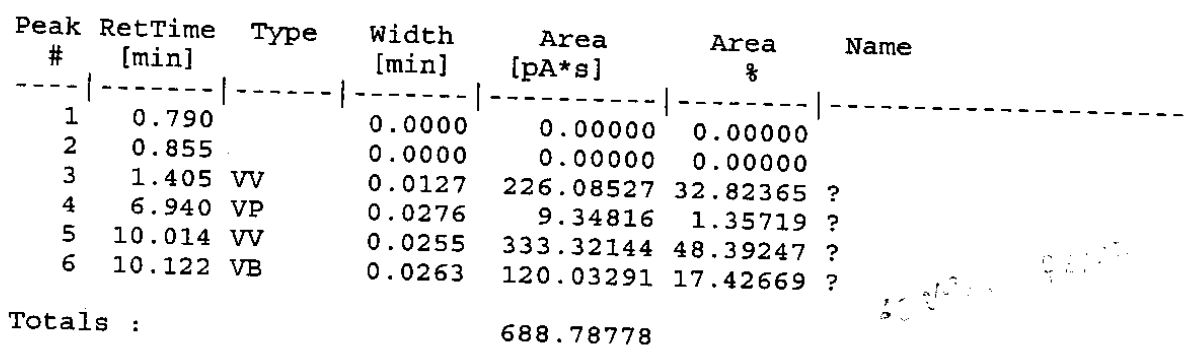

Results obtained with enhanced integrator!

2 Warnings or Errors :

Warning : Calibration warnings (see calibration table listing)

Warning : Calibrated compound(s) not found 

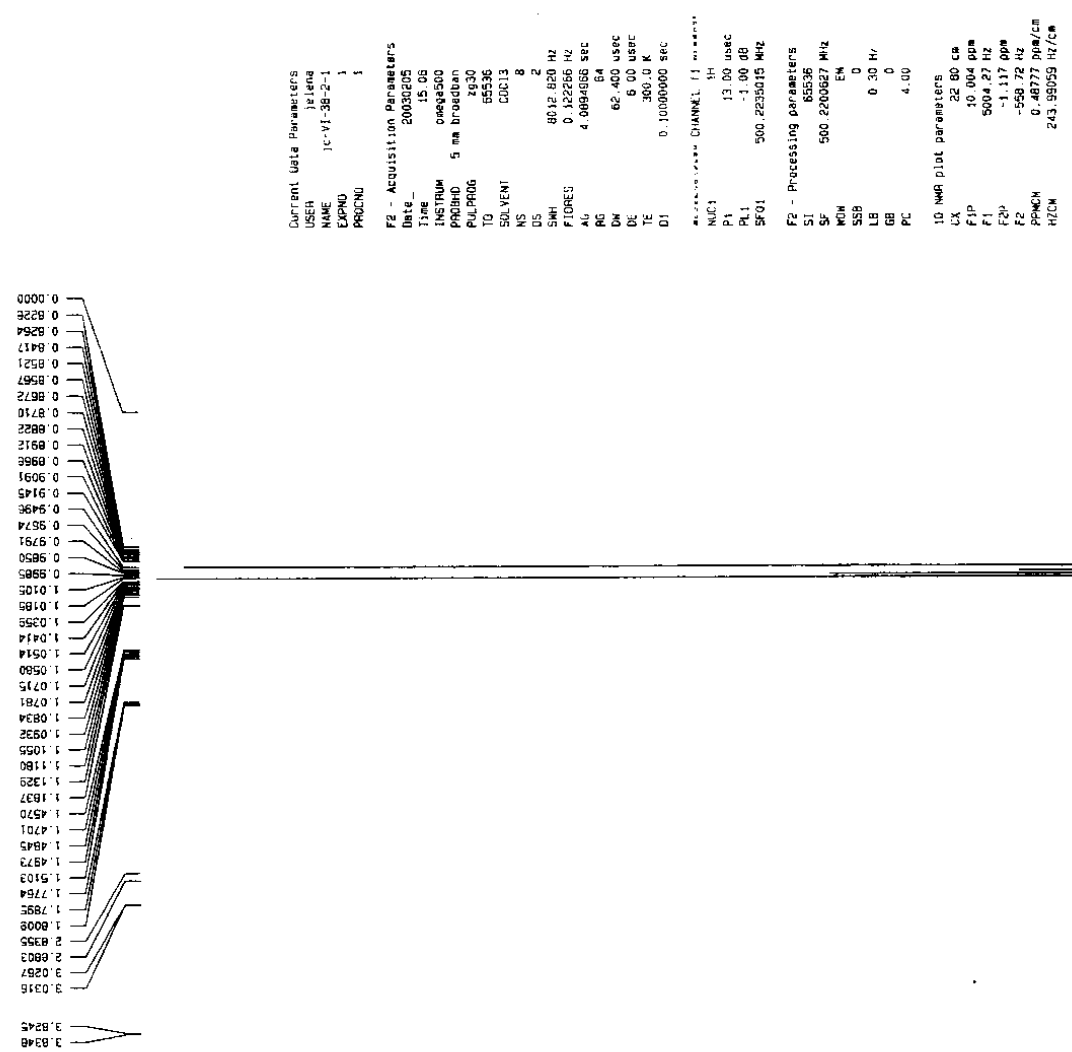

$54 \angle C$,
$8082 ;$
$826, ;$

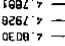

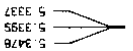

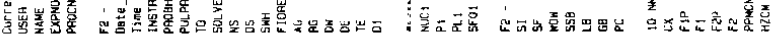
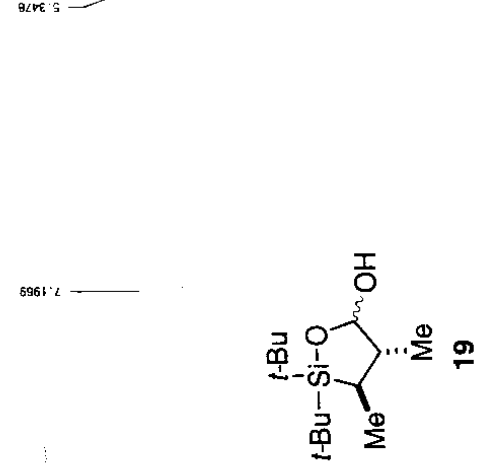

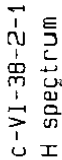
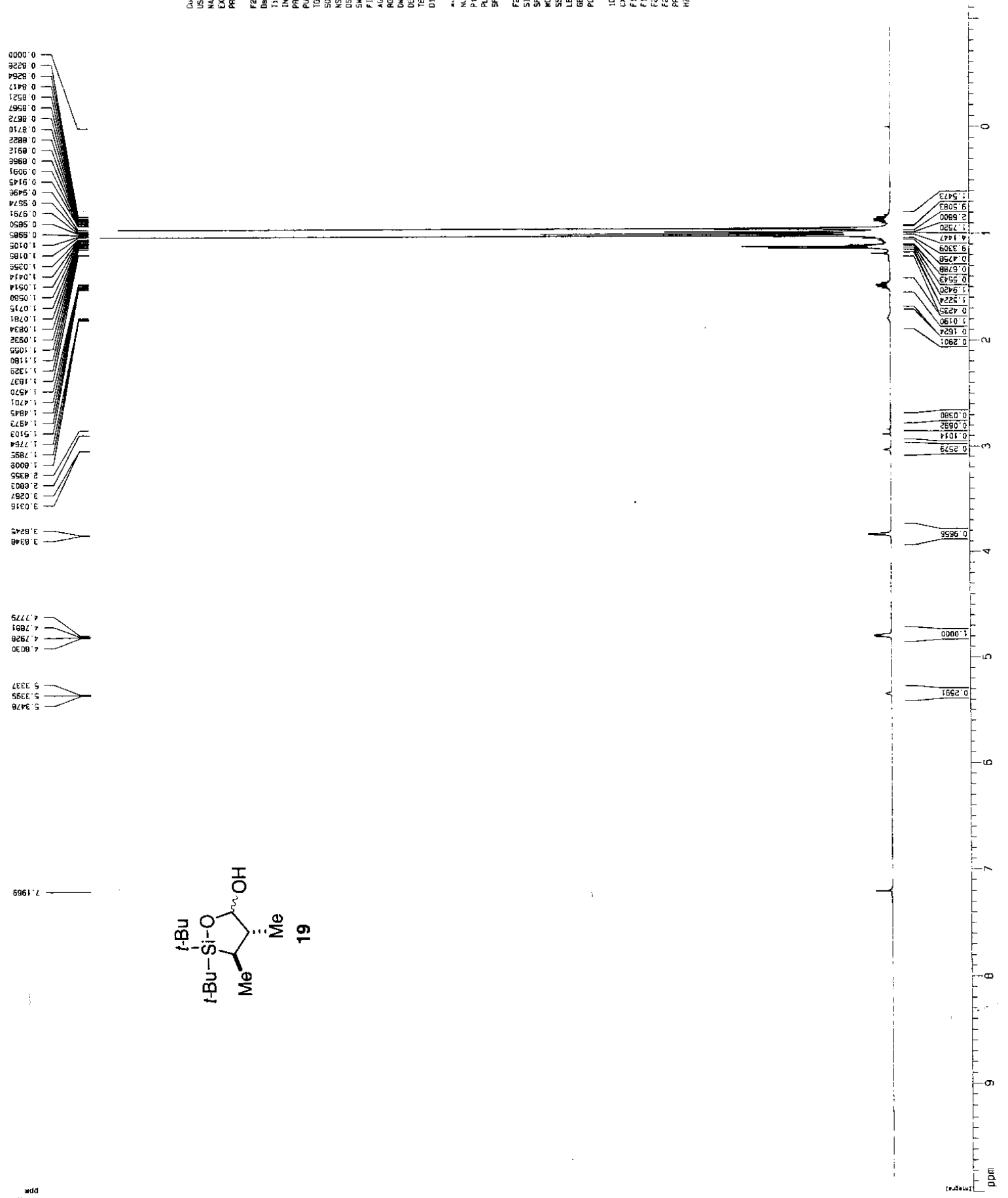


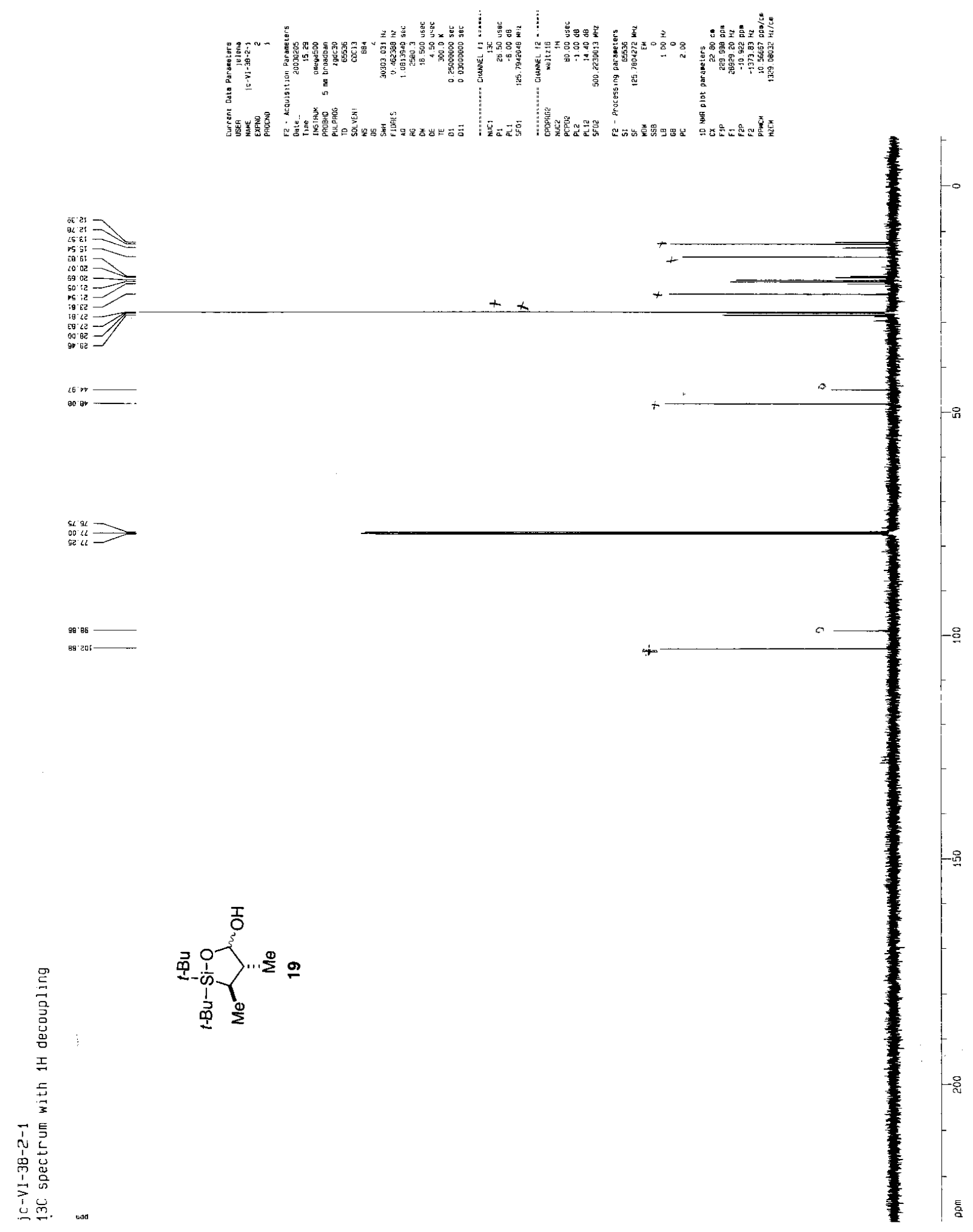




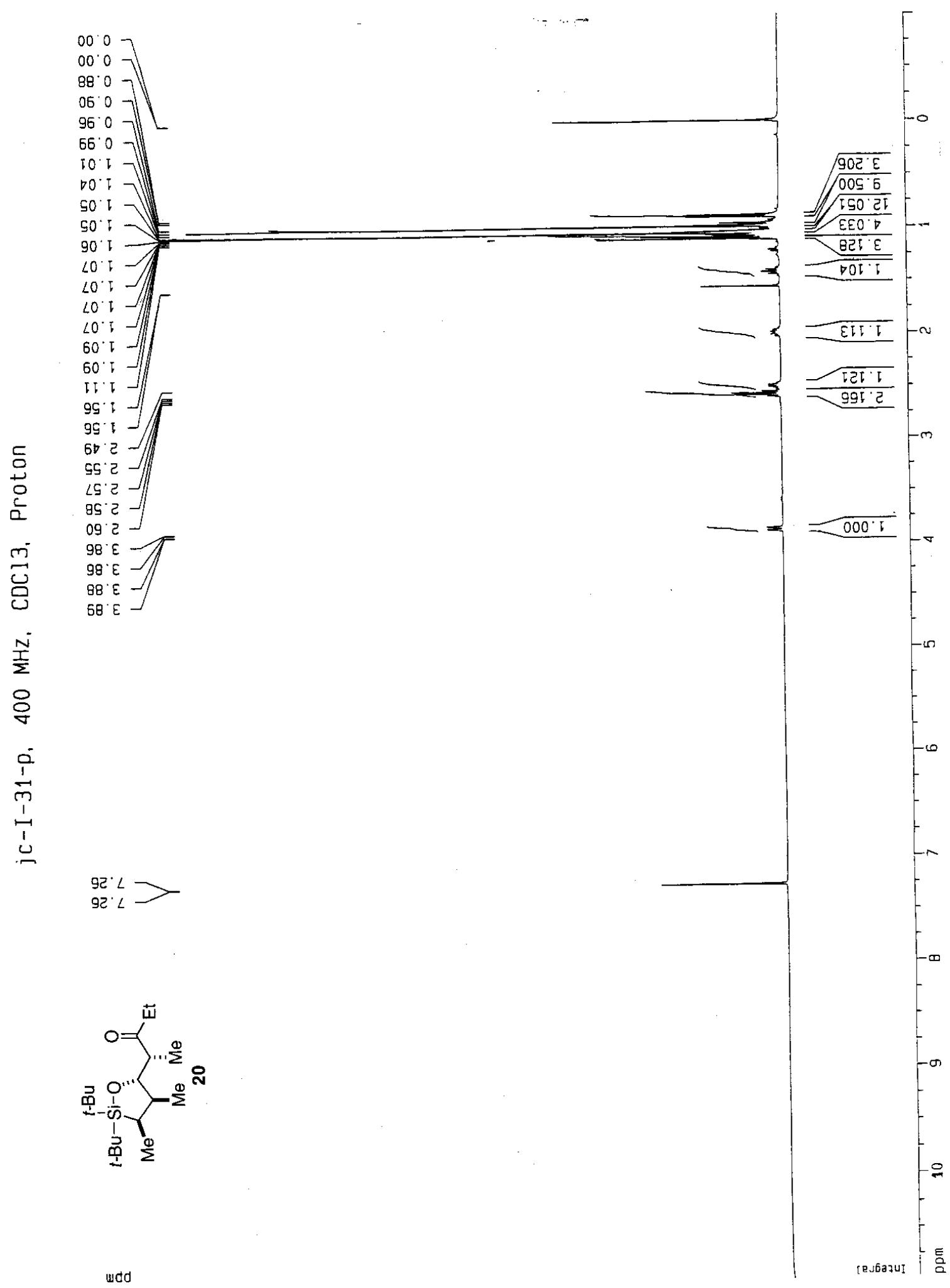




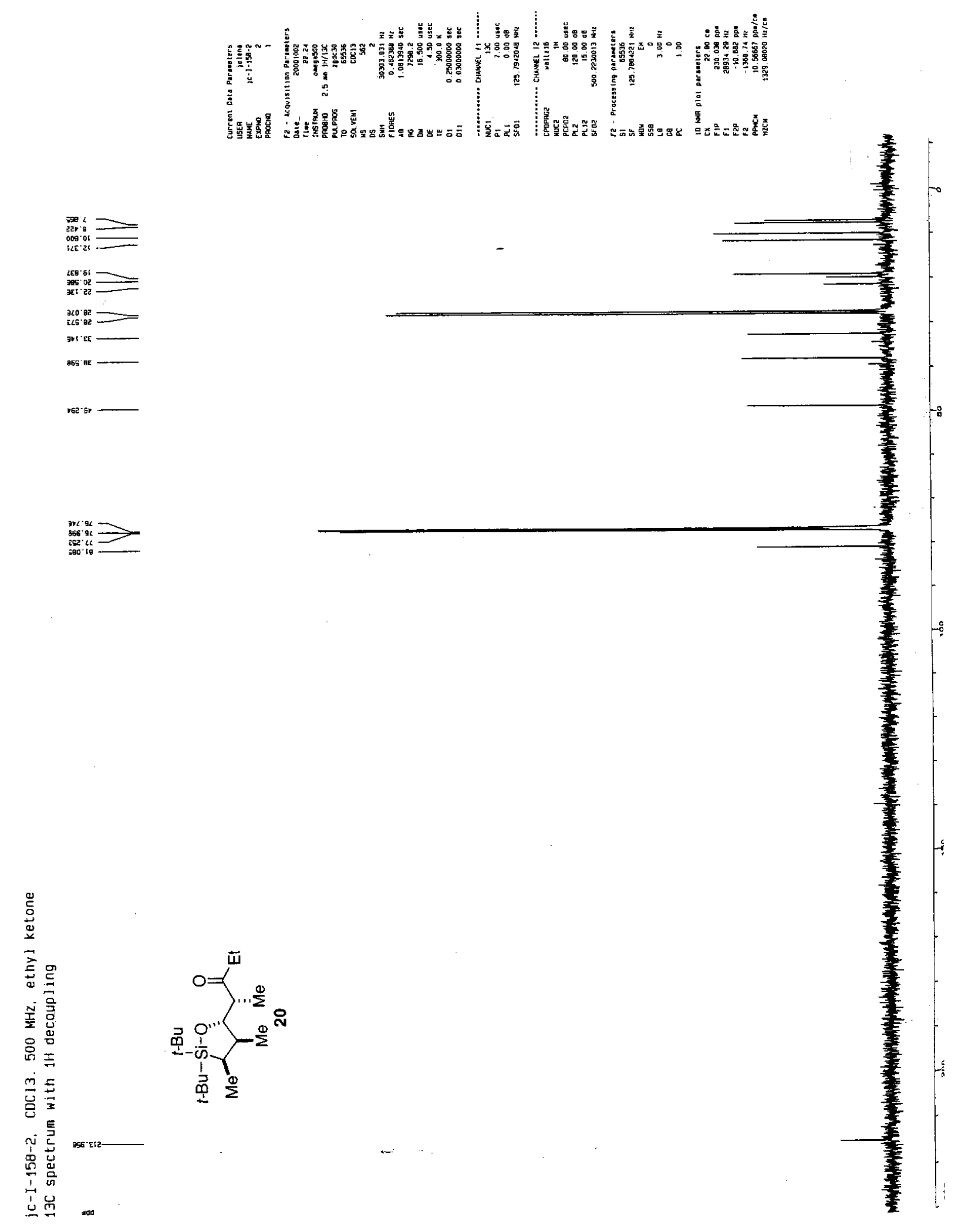




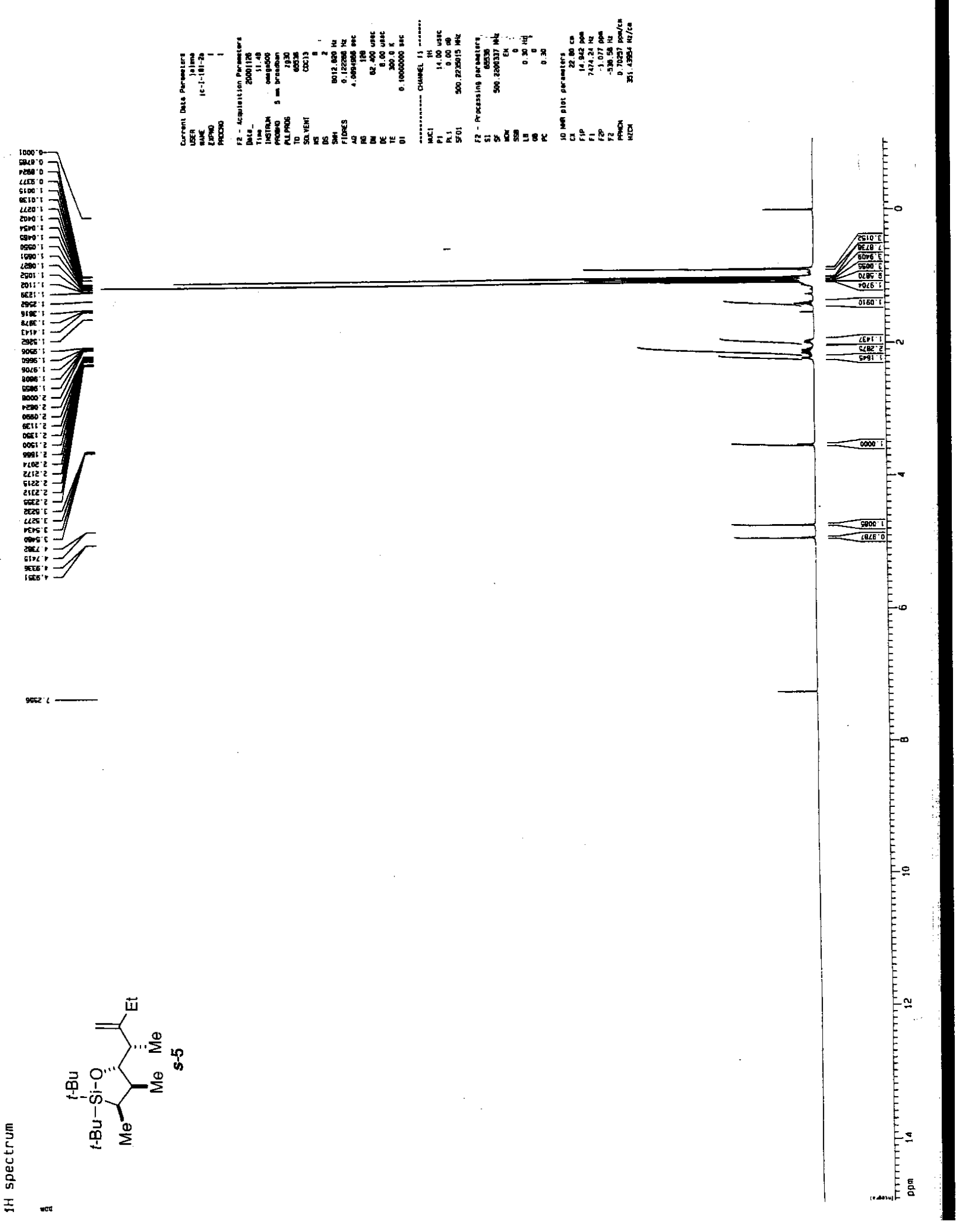



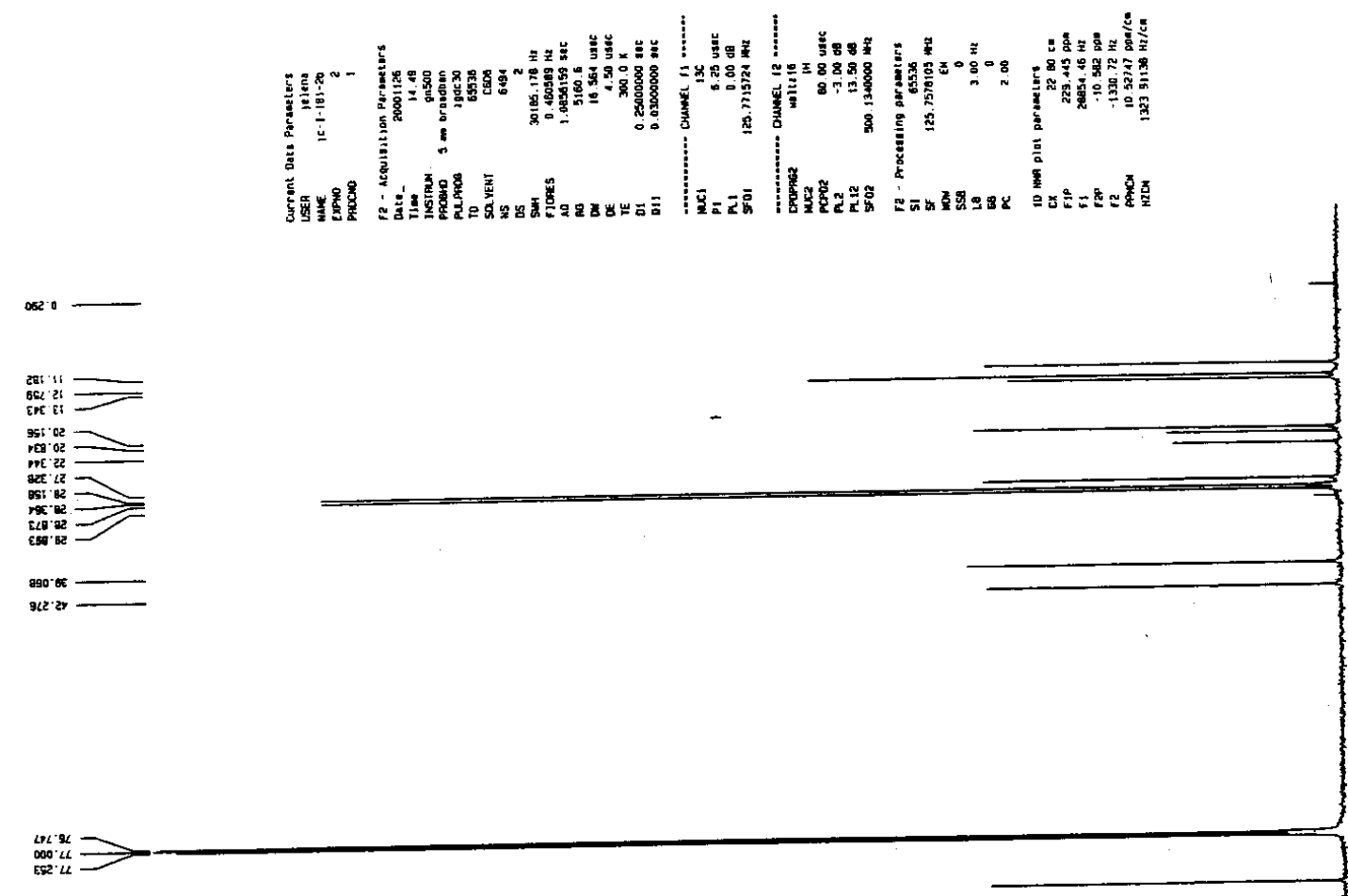

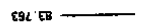

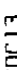

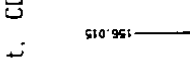

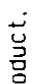

¿

$\stackrel{g}{ \pm}$

동 :웜

Nㅗㄹ

员点

穴

覀

$\stackrel{1}{1} 4$

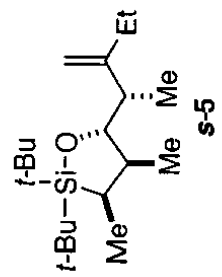

ad 

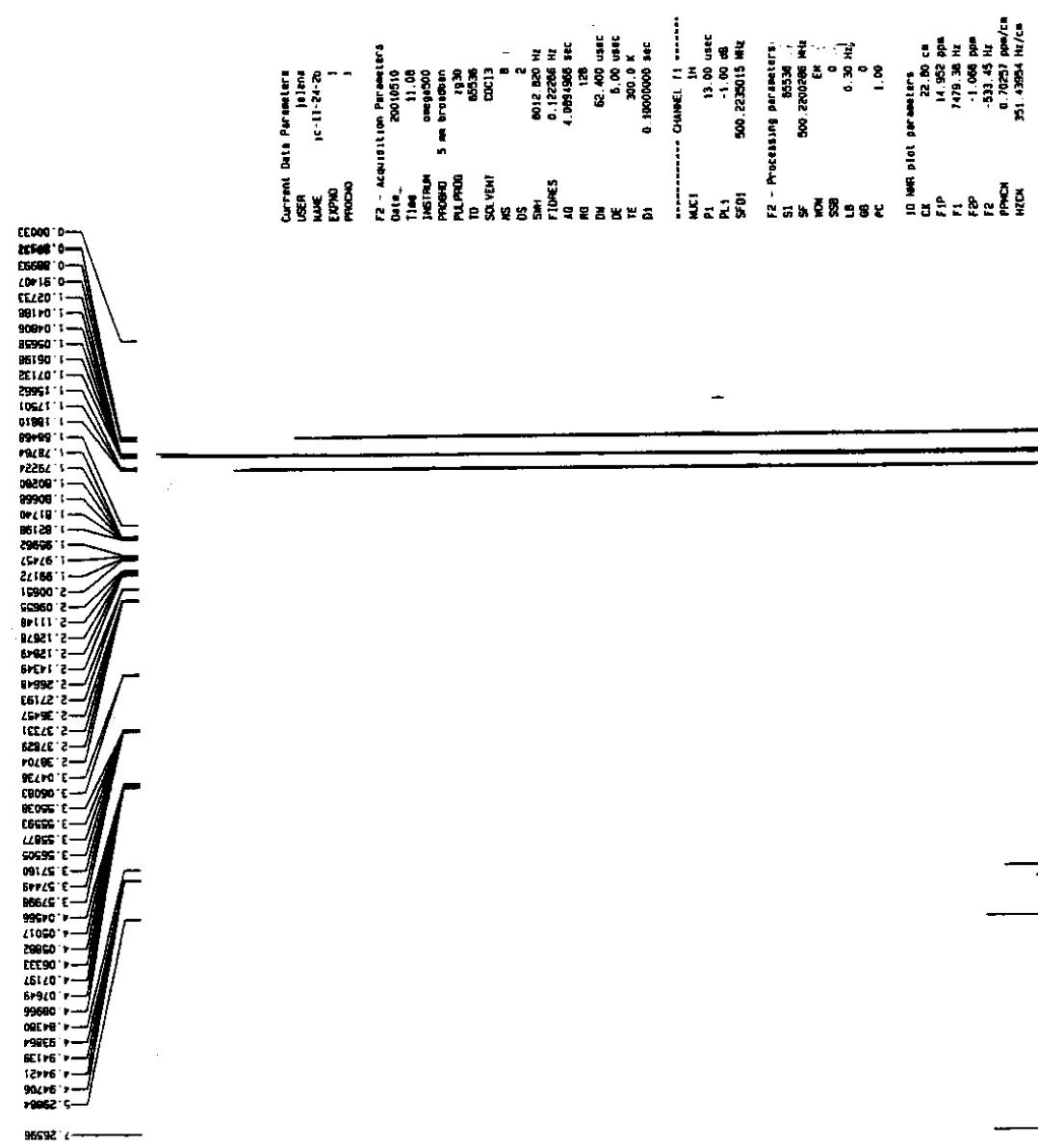

$$
=8
$$
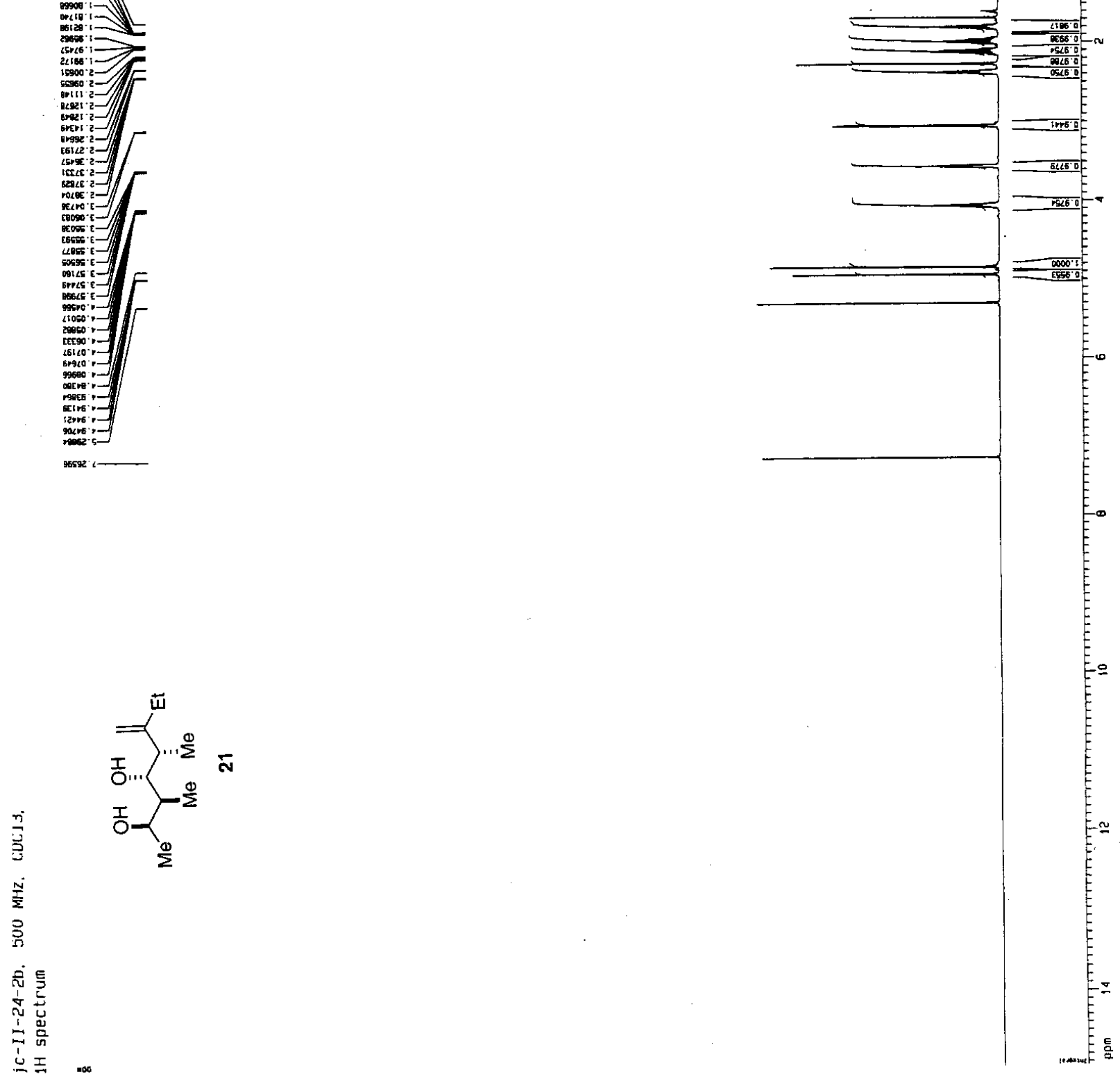

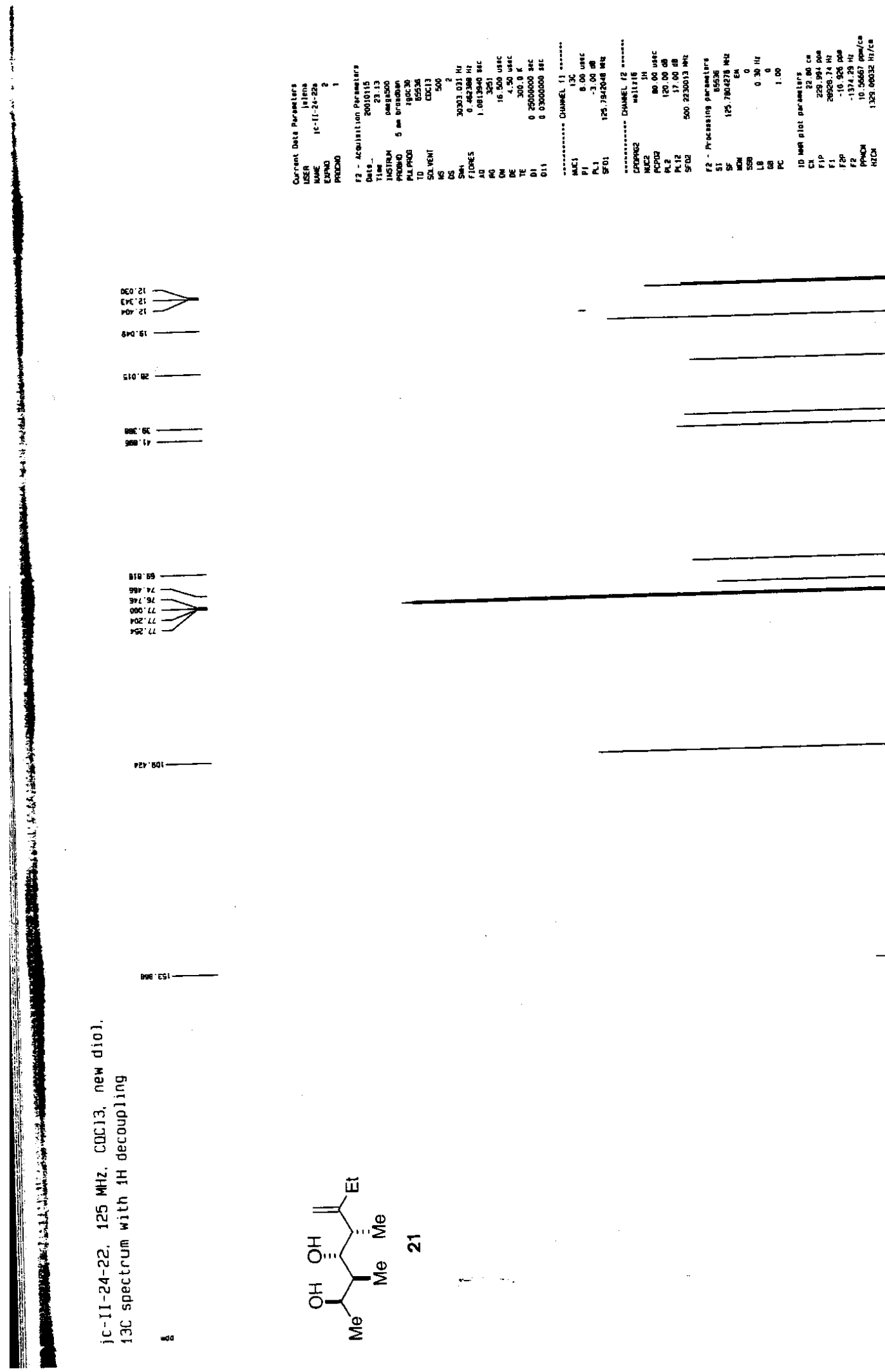